\title{
Enantioselective Vinylogous Mannich Reaction of Acyclic Vinylketene Silyl Acetals with Ketimines Using Chiral Bis(imidazoline)-Cu(II) Catalysts
}

\author{
Shuichi Nakamura ${ }^{[\mathrm{a}, \mathrm{b}] *}$, Koichi Matsuzaka, ${ }^{[\mathrm{a}]}$ Tsubasa Hatanaka, ${ }^{[\mathrm{c}]}$ Yasuhiro Funahashi $^{[\mathrm{c}]}$
}

[a] Department of Life Science and Applied Chemistry, Graduate School of Engineering, Nagoya Institute of Technology, Gokiso, Showa-ku, Nagoya 466-8555 (Japan), E-mail: snakamur@nitech.ac.jp; Tel \& Fax: 81-52-735-5245

[b] Frontier Research Institute for Material Science, Nagoya Institute of Technology

Gokiso, Showa-ku, Nagoya 466-8555 (Japan)

[c] Department of Chemistry, Graduate School of Science, Osaka University 1-1 Machikaneyama, Toyonaka, Osaka 560-0043 (Japan)

\section{CONTENTS:}

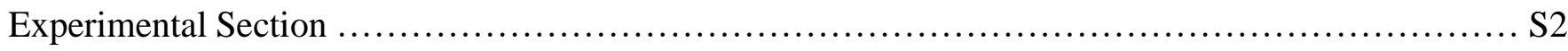

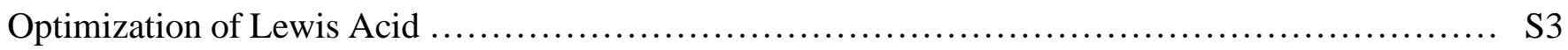

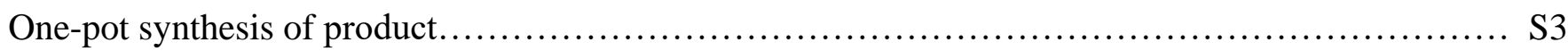

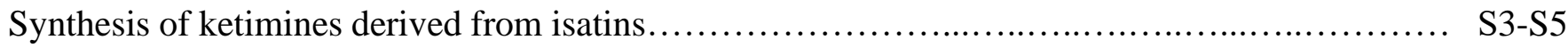

General procedure for the reaction of vinylketene silyl acetal to ketimines by chiral bis(imidazoline)$\mathrm{Cu}(\mathrm{II})$ catalyst

Characterization data of products.

ESI mass spectra for complexes.

References

${ }^{1} \mathrm{H},{ }^{13} \mathrm{C}$ NMR Spectra. S21-S51

HPLC Charts

S52-S72 


\section{Experimental Section}

General method: All reactions were performed in oven-dried glassware under a positive pressure of argon. Solvents were transferred via syringe and were introduced into the reaction vessels through a rubber septum. All reactions were monitored by thin-layer chromatography (TLC) carried out on $0.25 \mathrm{~mm}$ Merck silica-gel (60-F254). The TLC plates were visualized with UV light and 7\% phosphomolybdic acid or $p$ anisaldehyde in ethanol/heat. Column chromatography was carried out on a column packed with silicagel 60N spherical neutral size 63-210 $\mu \mathrm{m}$. The ${ }^{1} \mathrm{H}$ NMR (300 MHz) and ${ }^{19} \mathrm{~F} \mathrm{NMR}(282 \mathrm{MHz})$ spectra for solution in $\mathrm{CDCl}_{3}, \mathrm{CD}_{3} \mathrm{OD}$ or $\mathrm{CD}_{3} \mathrm{CN}$ were recorded on Varian Mercury 300, or ${ }^{1} \mathrm{H} \mathrm{NMR}(500 \mathrm{MHz})$ and ${ }^{13} \mathrm{C}$ NMR (125 MHz) spectrum for solution in $\mathrm{CDCl}_{3}$ or $\mathrm{CD}_{3} \mathrm{OD}$ were recorded on Bruker Avance 500. Chemical shifts $(\delta)$ are expressed in ppm downfield from internal TMS. HPLC analyses were performed on a JASCO LC-2000plus using 4.6 x 250 mm of CHIRALPAK ${ }^{\circledR}$ IA, IA-3, IB-3, IB N-3, IC-3, and IF-3 column. ESI Mass spectra were recorded on a Waters SYNAPT G2 HDMS. Optical rotations were measured on a JASCO P-2200. Infrared spectra were recorded on a JASCO FT/IR-4600 spectrometer with ZnSe ATR unit. The bis(imidazoline) was synthesized by published procedures. ${ }^{1)}$ Vinylketene silyl acetals were synthesized by published procedures. ${ }^{2}$ ) 


\section{Optimization of Lewis Acid}

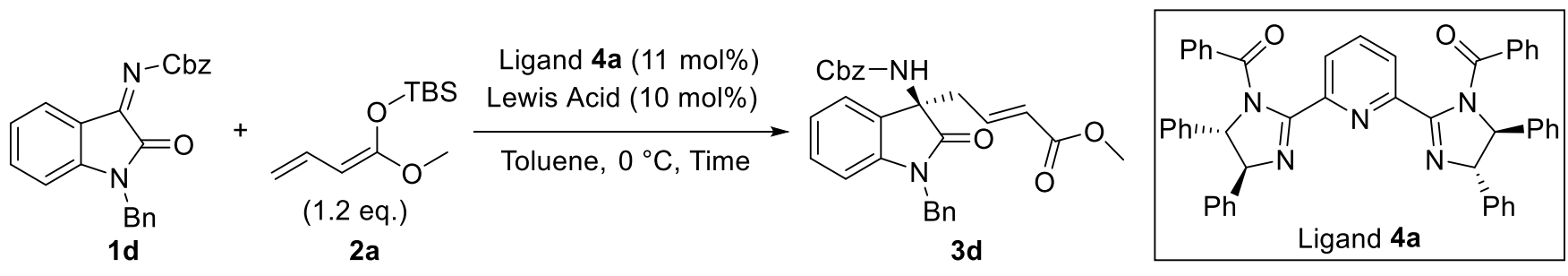

\begin{tabular}{ccccc}
\hline Entry & Lewis Acid & Time $(\mathrm{h})$ & Yield $(\%)$ & Ee $(\%)^{[a]}$ \\
\hline 1 & $\mathrm{Cu}(\mathrm{OTf})_{2}$ & 24 & 93 & $93(R)$ \\
2 & $\mathrm{Cu}\left(\mathrm{NTf}_{2}\right)_{2}$ & 25 & 82 & $47(R)$ \\
3 & $\mathrm{CuOTf} \cdot 1 / 2$ toluene & 25 & 98 & $89(R)$ \\
4 & $\mathrm{Cul}$ & 90 & 84 & 0 \\
5 & $\mathrm{Zn}(\mathrm{OTf})_{2}$ & 24 & 99 & $28(S)$ \\
6 & $\mathrm{In}(\mathrm{OTf})_{3}$ & 72 & 17 & $1(R)$ \\
\hline
\end{tabular}

\section{One-pot procedure for vinylogous Mannich reaction of $N$-benzyl isatin}

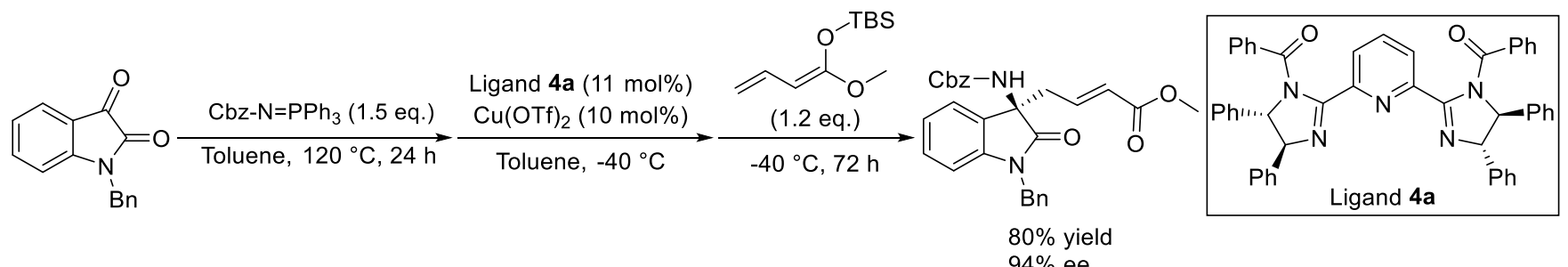

\section{General procedure for the synthesis of ketimines:}

Ketimines 1a-f, $\mathbf{h}, \mathbf{j}-\mathbf{l}$ are synthesized by previous reported method. ${ }^{\mathbf{3}}$

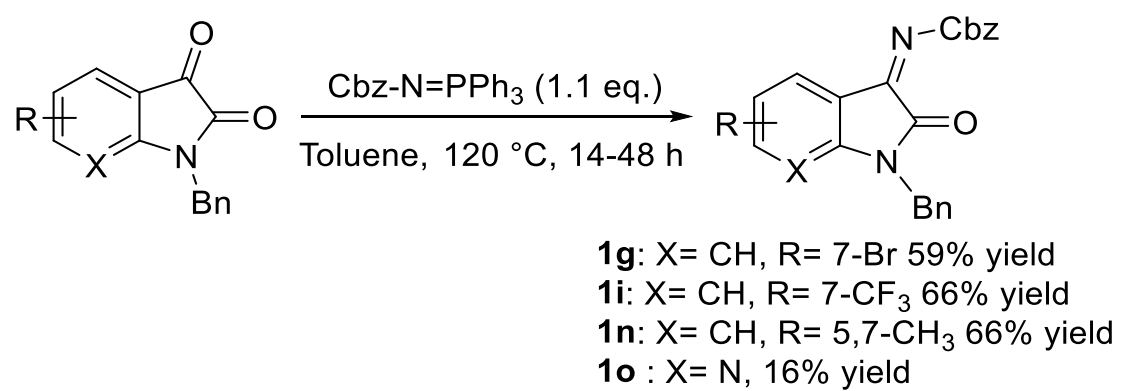

$N$-Benzyl isatin $(3 \mathrm{mmol})$ and $N$-benzyloxycarbonylaminotriphenylphosphine (1.46 g, $3.3 \mathrm{mmol})$ was dissolved in toluene $(3.0 \mathrm{~mL})$ at room temperature, and the reaction mixture was refluxed for $14-48 \mathrm{~h}$. Then the resulting mixture was cooling to room temperature, solvents were removed under reduced pressure. The crude residue was purified by flash silica gel chromatography (benzene : ethyl acetate $=99$ : 1), and recrystallized from hexane and ethyl acetate or diethyl ether to afford ketimine $\mathbf{1}$. 


\section{Benzyl 1-benzyl-7-bromo-2-oxoindolin-3-ylidenecarbamate (1g)}

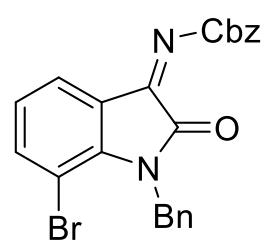

According to the general procedure, the reaction using $N$-benzyl 7-bromoisatin $(948.5$ $\mathrm{mg}, 3 \mathrm{mmol}$ ) and $\mathrm{N}$-benzyloxycarbonylaminotriphenylphosphine (1.46 g, $3.3 \mathrm{mmol})$ gave $1 \mathrm{~g}$ as a red solid (790.0 $\mathrm{mg}, 59 \%$ yield).

m.p. 92.2-92.8 ${ }^{\circ} \mathrm{C} ;{ }^{1} \mathrm{H}$ NMR $\left(300 \mathrm{MHz}, \mathrm{CDCl}_{3}\right) \delta 5.38$ (s, 4H), 6.96 (s, 1H), 7.22-7.45 $(\mathrm{m}, 10 \mathrm{H}), 7.55(\mathrm{~d}, J=8.1 \mathrm{~Hz}, 1 \mathrm{H}), 7.66(\mathrm{~s}, 1 \mathrm{H}) ;{ }^{13} \mathrm{C} \mathrm{NMR}\left(125 \mathrm{MHz}, \mathrm{CDCl}_{3}\right) \delta 44.7,69.2,103.7,122.3$, 123.9, 125.0, 126.5, 127.5, 128.6, 128.7, 128.7, 134.9, 136.0, 141.4, 144.6, 153.5, 158.0, 161.6; IR (ATR) 2948, 1730, 1685, 1610, 1345, 1209, 1185, 1124, 755, 722, $698 \mathrm{~cm}^{-1}$; HRMS (ESI, positive) m/z for $\mathrm{C}_{23} \mathrm{H}_{17} \mathrm{BrN}_{2} \mathrm{NaO}_{3}[\mathrm{M}+\mathrm{Na}]^{+}$: calcd. 471.0315, found 471.0321 .

\section{Benzyl 1-benzyl-7-trifluoromethyl-2-oxoindolin-3-ylidenecarbamate (1i)}

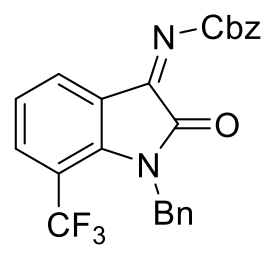

According to the general procedure, the reaction using $N$-benzyl 7-trifluoromethylisatin (915.8 $\mathrm{mg}, 3 \mathrm{mmol})$ and $N$-benzyloxycarbonylaminotriphenylphosphine (1.46 g, 3.3 mmol) gave $1 \mathrm{i}$ as a yellow solid (865.4 $\mathrm{mg}, 66 \%$ yield).

m.p. 105.0-105.8 ${ }^{\circ} \mathrm{C} ;{ }^{1} \mathrm{H}$ NMR (300 $\left.\mathrm{MHz} \mathrm{CDCl}_{3}\right) \delta 5.18$ (s, 2H), 5.37 (s, 2H), 7.12 (d, $J=7.8 \mathrm{~Hz}, 2 \mathrm{H}), 7.21-7.42(\mathrm{~m}, 9 \mathrm{H}), 7.42(\mathrm{~d}, J=4.5 \mathrm{~Hz}, 1 \mathrm{H}), 7.76(\mathrm{~d}, J=8.1 \mathrm{~Hz}, 1 \mathrm{H}), 7.92(\mathrm{~s}, 1 \mathrm{H}) ;{ }^{13} \mathrm{C}$ NMR $\left(125 \mathrm{MHz}, \mathrm{CDCl}_{3}\right) \delta 46.0-46.1(\mathrm{~m}), 69.3,114.4\left(\mathrm{q}, J_{C-F}=33.4 \mathrm{~Hz}\right), 119.3,121.5,121.7,123.4$, $123.7,125.8,127.4,128.1,128.6\left(\mathrm{~d}, J_{C-F}=3.5 \mathrm{~Hz}\right), 128.8,133.4-133.5(\mathrm{~m}), 134.8,134.9,145.4\left(\mathrm{~d}, J_{C-F}\right.$ $=2.0 \mathrm{~Hz}$ ), 152.5, 158.3, 161.5; IR (ATR) 2951, 1730, 1685, 1610, 1345, 1209, 1185, 1124, 755, 722, 698 $\mathrm{cm}^{-1}$; HRMS (ESI, positive) $\mathrm{m} / \mathrm{z}$ for $\mathrm{C}_{24} \mathrm{H}_{17} \mathrm{~F}_{3} \mathrm{~N}_{2} \mathrm{NaO}_{3}[\mathrm{M}+\mathrm{Na}]^{+}$: calcd. 461.1083, found 461.1081.

\section{Benzyl 1-benzyl-5,7-dimethyl-2-oxoindolin-3-ylidenecarbamate (1n)}<smiles>Cc1cc(C)c2c(c1)/C(=N/C(=O)OCc1ccccc1)C(=O)N2Cc1ccccc1</smiles>

According to the general procedure, the reaction using $N$-benzyl 5,7dimethylisatin (795.3 $\mathrm{mg}, 3 \mathrm{mmol})$ and $N$-benzyloxycarbonylamino triphenylphosphine (1.46 g, $3.3 \mathrm{mmol}$ ) gave $1 \mathrm{n}$ as a red solid (787.2 $\mathrm{mg}, 66 \%$ yield). m.p. 163.5-164.2 ${ }^{\circ} \mathrm{C} ;{ }^{1} \mathrm{H}$ NMR (300 MHz, $\left.\mathrm{CDCl}_{3}\right) \delta 2.17$ (s, 3H), $2.23(\mathrm{~s}, 3 \mathrm{H}), 5.11$ $(\mathrm{s}, 2 \mathrm{H}), 5.38(\mathrm{~s}, 2 \mathrm{H}), 6.95(\mathrm{~s}, 1 \mathrm{H}), 7.16(\mathrm{~d}, J=8.1 \mathrm{~Hz}, 2 \mathrm{H}), 7.23-7.46(\mathrm{~m}, 9 \mathrm{H}) ;{ }^{13} \mathrm{C}$ NMR $(125 \mathrm{MHz}$, $\left.\mathrm{CDCl}_{3}\right) \delta 18.3,20.4,45.2,68.9,119.9,121.1,123.2,125.7,127.5,128.6,128.7,129.0,133.5,135.2,136.3$, 140.5, 143.2, 154.9, 158.6, 162.2; IR (ATR) 2966, 1727, 1714, 1682, 1601, 1347, 1208, 1158, 989, 728, $695 \mathrm{~cm}^{-1}$; HRMS (ESI, positive) $\mathrm{m} / \mathrm{z}$ for $\mathrm{C}_{25} \mathrm{H}_{22} \mathrm{~N}_{2} \mathrm{NaO}_{3}[\mathrm{M}+\mathrm{Na}]^{+}$: calcd. 421.1523, found 421.1526. 
Benzyl 1-benzyl-2-oxo-1,2-dihydro-3H-pyrrolo[2,3-b]pyridin-3-ylidenecarbamate (10)

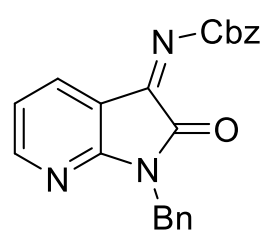

According to the general procedure, the reaction using $N$-benzyl azaisatin $(405.0 \mathrm{mg}$, $1.7 \mathrm{mmol})$ and $N$-benzyloxycarbonylaminotriphenylphosphine $(782.9 \mathrm{mg}, 1.9 \mathrm{mmol})$ gave 10 as a yellow solid (102.5 $\mathrm{mg}, 16 \%$ yield).

m.p. 119.4-120.4 ${ }^{\circ} \mathrm{C} ;{ }^{1} \mathrm{H} \mathrm{NMR}\left(300 \mathrm{MHz}, \mathrm{CDCl}_{3}\right) \delta 4.98$ (s, 2H), 5.39 (s, 2H), 7.04 (s, 1H), 7.27-7.49 (m, 10H), 7.86 (s, 1H), $8.36(\mathrm{~d}, J=2.1 \mathrm{~Hz}, 1 \mathrm{H}) ;{ }^{13} \mathrm{C} \mathrm{NMR}\left(125 \mathrm{MHz}, \mathrm{CDCl}_{3}\right) \delta 42.8,69.2$, 113.8, 119.4, 128.0, 128.6, 128.7, 128.7, 128.8, 132.1, 134.9, 135.4, 153.2, 153.6, 157.0, 160.5, 161.4; IR (ATR) 2933, 1720, 1682, 1592, 1444, 1220, 1170, 1095, 966, 753, $696 \mathrm{~cm}^{-1}$; HRMS (ESI, positive) m/z for $\mathrm{C}_{22} \mathrm{H}_{17} \mathrm{~N}_{3} \mathrm{NaO}_{3}[\mathrm{M}+\mathrm{Na}]^{+}$: calcd. 394.1162, found 394.1161.

General procedure for the enantioselective vinylogous Mannich reaction of ketimines with acyclic vinylketene silyl acetals:

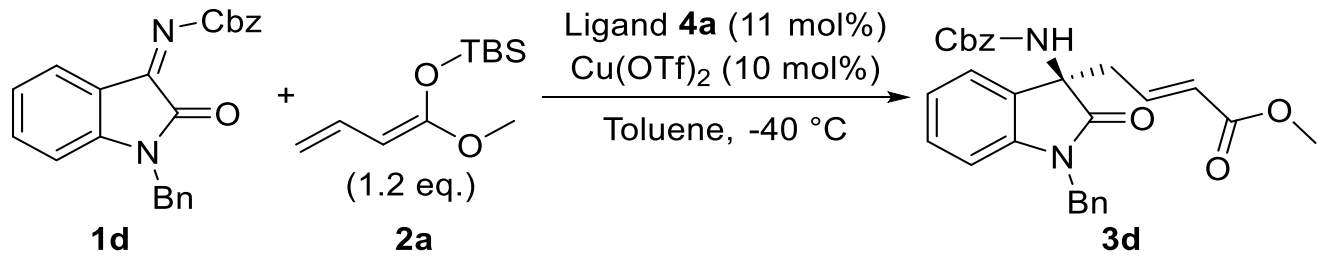

A solution of $4 \mathbf{a}(0.011 \mathrm{mmol}, 11 \mathrm{~mol} \%)$ and $\mathrm{Cu}(\mathrm{OTf})_{2}(0.01 \mathrm{mmol}, 10 \mathrm{~mol} \%)$ in dry toluene $(1.0 \mathrm{~mL})$ was stirred at room temperature for $1 \mathrm{~h}$. Ketimine $1 \mathrm{~d}(0.10 \mathrm{mmol})$ was added to the mixture and stirred at $-40{ }^{\circ} \mathrm{C}$. Vinylketene silyl acetal $\mathbf{2 a}(0.12 \mathrm{mmol})$ was added. After completion of the reaction monitored by TLC, the reaction mixture was concentrated and purified by silica gel column chromatography (hexane $/ \mathrm{CH}_{2} \mathrm{Cl}_{2} /$ ethyl acetate $=4: 1: 1$ ) to afford $\mathbf{3 d}$.

\section{Methyl (R,E)-4-\{3-[(tert-butoxycarbonyl)amino]-2-oxoindolin-3-yl\}but-2-enoate (3a)}

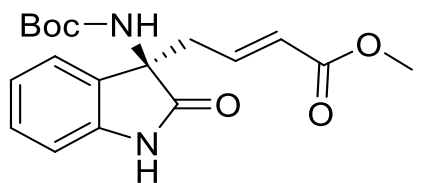

Reaction of ketimine $1 \mathbf{a}(0.10 \mathrm{mmol})$ and vinylketene silyl acetal $\mathbf{2 a}(0.12$ $\mathrm{mmol})$ using $4 \mathrm{a}(0.01 \mathrm{mmol})$ and $\mathrm{Cu}(\mathrm{OTf})_{2}(0.01 \mathrm{mmol})$ in toluene $(1.00 \mathrm{~mL})$ at $0{ }^{\circ} \mathrm{C}$ for $24 \mathrm{~h}$ gave $(R)-3 \mathbf{a}(15.9 \mathrm{mg}, 46 \%$ yield, $8 \%$ ee) as a white solid.

$[\alpha]_{\mathrm{D}}{ }^{25}-0.9$ (c 0.28, $\left.\mathrm{CHCl}_{3}\right)$; m.p. 181.2-182.0 ${ }^{\circ} \mathrm{C} ;{ }^{1} \mathrm{H}$ NMR $\left(300 \mathrm{MHz}, \mathrm{CDCl}_{3}\right) \delta 1.28(\mathrm{~s}, 9 \mathrm{H}), 2.63(\mathrm{dd}$, $J=7.8,13.8 \mathrm{~Hz}, 1 \mathrm{H}), 2.76(\mathrm{dd}, J=7.8,13.8 \mathrm{~Hz}, 1 \mathrm{H}), 3.73(\mathrm{~s}, 3 \mathrm{H}), 5.27(\mathrm{~s}, 1 \mathrm{H}), 5.94(\mathrm{~d}, J=15.6 \mathrm{~Hz}, 1 \mathrm{H})$, 6.76-6.84 (m, 1H), $6.87(\mathrm{~d}, J=7.5 \mathrm{~Hz}, 1 \mathrm{H}), 7.07(\mathrm{t}, J=7.5 \mathrm{~Hz}, 1 \mathrm{H}), 7.23-7.27(\mathrm{~m}, 2 \mathrm{H}), 8.09(\mathrm{br}, 1 \mathrm{H}) ;{ }^{13} \mathrm{C}$ NMR $\left(125 \mathrm{MHz}, \mathrm{CDCl}_{3}\right) \delta$ 28.0, 39.9, 51.7, 61.2, 81.0, 110.4, 122.8, 123.2, 126.4, 129.1, 130.1, 139.8, 140.1, 153.9, 166.0, 178.1; IR (ATR) 3290, 2978, 1716, 1683, 1621, 1516, 1280, 1158, 1029, 966, 754 $\mathrm{cm}^{-1}$; HRMS (ESI, positive) $\mathrm{m} / \mathrm{z}$ for $\mathrm{C}_{18} \mathrm{H}_{22} \mathrm{~N}_{2} \mathrm{NaO}_{5}[\mathrm{M}+\mathrm{Na}]^{+}$: calcd. 369.1421, found 369.1414; HPLC (DAICEL CHIRALPAK IA-3®, hexane: $i$-PrOH $=80: 20,1.0 \mathrm{~mL} / \mathrm{min}, 254 \mathrm{~nm}$ ), $\mathrm{tR}=9.6 \mathrm{~min}$ (major), 
$18.9 \min$ (minor).

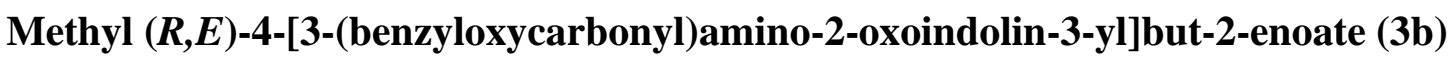

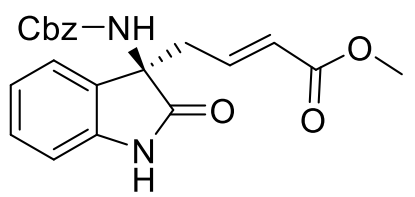

Reaction of ketimine $\mathbf{1 b}(0.10 \mathrm{mmol})$ and vinylketene silyl acetal 2a $(0.12$ $\mathrm{mmol})$ using $4 \mathbf{a}(0.01 \mathrm{mmol})$ and $\mathrm{Cu}(\mathrm{OTf})_{2}(0.01 \mathrm{mmol})$ in toluene $(1.0 \mathrm{~mL})$ at $0{ }^{\circ} \mathrm{C}$ for $24 \mathrm{~h}$ gave $(R)-3 \mathbf{b}(37.6 \mathrm{mg}, 99 \%$ yield, $66 \%$ ee) as a white solid.

$[\alpha]_{\mathrm{D}}{ }^{25}-17.9$ (c 0.36, $\left.\mathrm{CHCl}_{3}\right)$; m.p. 123.6-124.4 ${ }^{\circ} \mathrm{C} ;{ }^{1} \mathrm{H}$ NMR $\left(300 \mathrm{MHz}, \mathrm{CDCl}_{3}\right) \delta 2.62(\mathrm{dd}, J=7.7,13.8$ Hz, 1H), 2.78 (dd, J = 7.7, 13.8 Hz, 1H), 3.69 (s, 3H), 4.97 (s, 2H), 5.86-5.94 (m, 2H), 6.76-6.84 (m, 2H), $7.05(\mathrm{t}, J=7.5 \mathrm{~Hz}, 1 \mathrm{H}), 7.20-7.26(\mathrm{~m}, 7 \mathrm{H}), 8.66(\mathrm{br}, 1 \mathrm{H}) ;{ }^{13} \mathrm{C} \mathrm{NMR}\left(125 \mathrm{MHz}, \mathrm{CDCl}_{3}\right) \delta 39.6,51.8,61.4$, $67.4,110.7,122.9,123.4,126.5,128.1,128.2$, 128.5, 129.3, 135.6, 139.7, 140.4; 154.6, 166.0, 177.8; IR (ATR) 3285, 2950, 1716, 1618, 1509, 1470, 1203, 1038, 967, 739, $697 \mathrm{~cm}^{-1}$; HRMS (ESI, positive) m/z for $\mathrm{C}_{21} \mathrm{H}_{20} \mathrm{~N}_{2} \mathrm{NaO}_{5}[\mathrm{M}+\mathrm{Na}]^{+}$: calcd. 403.1264, found 403.1266; HPLC (DAICEL CHIRALPAK IC-3®, hexane: $i-\mathrm{PrOH}=80: 20,1.0 \mathrm{~mL} / \mathrm{min}, 254 \mathrm{~nm}$ ), $\mathrm{tR}=23.7 \mathrm{~min}$ (minor), $32.6 \mathrm{~min}$ (major).

\section{Methyl (R,E)-4-[3-(benzyloxycarbonyl)amino-1-methyl-2-oxoindolin-3-yl]but-2-enoate (3c)}

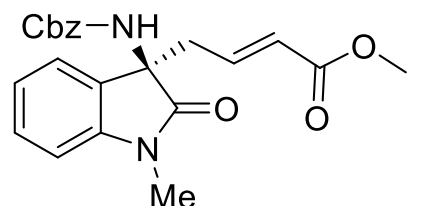

Reaction of ketimine 1c $(0.10 \mathrm{mmol})$ and vinylketene silyl acetal 2a $(0.12$ mmol) using $4 \mathbf{a}(0.01 \mathrm{mmol})$ and $\mathrm{Cu}(\mathrm{OTf})_{2}(0.01 \mathrm{mmol})$ in toluene $(1.0 \mathrm{~mL})$ at $0{ }^{\circ} \mathrm{C}$ for $24 \mathrm{~h}$ gave $(R)-3 \mathrm{c}(35.1 \mathrm{mg}, 89 \%$ yield, $85 \%$ ee) as a white solid.

$[\alpha]_{\mathrm{D}}{ }^{25}-3.3\left(\mathrm{c} 0.32, \mathrm{CHCl}_{3}\right)$; m.p. 103.2-104.0 ${ }^{\circ} \mathrm{C} ;{ }^{1} \mathrm{H}$ NMR $\left(300 \mathrm{MHz}, \mathrm{CDCl}_{3}\right) \delta 2.63(\mathrm{dd}, J=7.8,14.0$ $\mathrm{Hz}, 1 \mathrm{H}), 2.78$ (dd, $J=7.8,14.0 \mathrm{~Hz}, 1 \mathrm{H}), 3.18$ (br, 3H), 3.71 (s, 3H), 4.93 (s, 2H), 5.44 (s, 1H), 5.91 (d, $J$ $=15.6 \mathrm{~Hz}, 1 \mathrm{H}), 6.71-6.82(\mathrm{~m}, 2 \mathrm{H}), 7.10(\mathrm{t}, J=7.5 \mathrm{~Hz}, 1 \mathrm{H}), 7.29-7.36(\mathrm{~m}, 7 \mathrm{H}) ;{ }^{13} \mathrm{C} \mathrm{NMR}(125 \mathrm{MHz}$, $\left.\mathrm{CDCl}_{3}\right) \delta 26.5,39.8,51.7,60.9,67.4,108.6,123.0,123.1,126.5,128.3,128.3,128.5,129.0,129.4,135.5$, 139.7, 143.2, 154.4, 165.9, 175.6; IR (ATR) 3311, 3033, 2951, 1704, 1613, 1470, 1252, 1028, 967, 752, $696 \mathrm{~cm}^{-1}$; HRMS (ESI, positive) $\mathrm{m} / \mathrm{z}$ for $\mathrm{C}_{22} \mathrm{H}_{22} \mathrm{~N}_{2} \mathrm{NaO}_{5}[\mathrm{M}+\mathrm{Na}]^{+}$: calcd. 417.1421, found 417.1419; HPLC (DAICEL CHIRALPAK IC-3®, hexane: $i-\mathrm{PrOH}=70: 30,1.0 \mathrm{~mL} / \mathrm{min}, 254 \mathrm{~nm}$ ), tR = $29.5 \mathrm{~min}$ (minor), $52.2 \mathrm{~min}$ (major).

\section{Methyl (R,E)-4-[1-benzyl-3-(benzyloxycarbonylamino)-2-oxoindolin-3-yl]but-2-enoate (3d)}

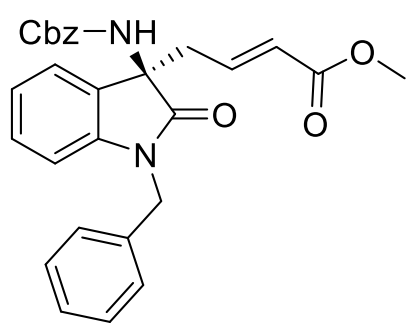

Reaction of ketimine $\mathbf{1 d}(0.10 \mathrm{mmol})$ and vinylketene silyl acetal $\mathbf{2 a}(0.12$ mmol) using $4 \mathbf{a}(0.01 \mathrm{mmol})$ and $\mathrm{Cu}(\mathrm{OTf})_{2}(0.01 \mathrm{mmol})$ in toluene $(1.0 \mathrm{~mL})$ at $-40{ }^{\circ} \mathrm{C}$ for $72 \mathrm{~h}$ gave $(R)-3 d$ ( $47.1 \mathrm{mg}, 99 \%$ yield, $97 \%$ ee) as a white solid. $[\alpha]_{\mathrm{D}}{ }^{25}-0.7$ (c 1.38, $\left.\mathrm{CHCl}_{3}\right)$; m.p. 47.0-48.0 ${ }^{\circ} \mathrm{C} ;{ }^{1} \mathrm{H} \mathrm{NMR}\left(300 \mathrm{MHz}, \mathrm{CDCl}_{3}\right) \delta$ $2.70(\mathrm{dd}, J=7.8,13.5 \mathrm{~Hz}, 1 \mathrm{H}), 2.83(\mathrm{dd}, J=7.8,13.5 \mathrm{~Hz}, 1 \mathrm{H}), 3.68(\mathrm{~s}, 3 \mathrm{H})$, $4.97(\mathrm{br}, 4 \mathrm{H}), 5.72(\mathrm{~s}, 1 \mathrm{H}), 5.91(\mathrm{~d}, J=15.6 \mathrm{~Hz}, 1 \mathrm{H}), 6.63-6.76(\mathrm{~m}, 2 \mathrm{H}), 7.05(\mathrm{t}, J=7.5 \mathrm{~Hz}, 1 \mathrm{H}), 7.16-$ 
$7.35(\mathrm{~m}, 12 \mathrm{H}) ;{ }^{13} \mathrm{C} \mathrm{NMR}\left(125 \mathrm{MHz}, \mathrm{CDCl}_{3}\right) \delta 40.0,44.1,51.7,61.0,67.4,109.7,123.0,123.1,126.6$, 127.1, 127.5, 128.3, 128.5, 128.8, 129.3, 135.4, 135.6, 139.5, 142.4, 154.3, 165.8, 175.7; IR (ATR) 3319, 3033, 2952, 1706, 1613, 1489, 1258, 1178, 1028, 750, $696 \mathrm{~cm}^{-1}$; HRMS (ESI, positive) m/z for $\mathrm{C}_{28} \mathrm{H}_{26} \mathrm{~N}_{2} \mathrm{NaO}_{5}[\mathrm{M}+\mathrm{Na}]^{+}$: calcd. 493.1734, found 493.1748; HPLC (DAICEL CHIRALPAK IF-3®, hexane: $i-\mathrm{PrOH}=70: 30,1.0 \mathrm{~mL} / \mathrm{min}, 254 \mathrm{~nm}$ ), $\mathrm{tR}=13.7 \mathrm{~min}$ (major), $33.1 \mathrm{~min}$ (minor).

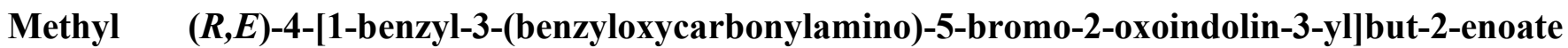
(3e)

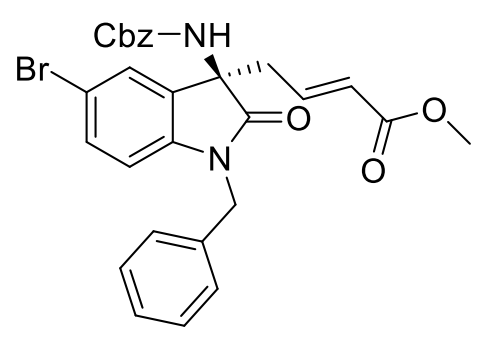

Reaction of ketimine $1 \mathbf{e}(0.10 \mathrm{mmol})$ and vinylketene silyl acetal $\mathbf{2 a}(0.12$ $\mathrm{mmol})$ using $4 \mathbf{a}(0.01 \mathrm{mmol})$ and $\mathrm{Cu}(\mathrm{OTf})_{2}(0.01 \mathrm{mmol})$ in toluene $(1.0 \mathrm{~mL})$ at $-40{ }^{\circ} \mathrm{C}$ for $96 \mathrm{~h}$ gave $(R)-3 \mathrm{e}(54.4 \mathrm{mg}, 99 \%$ yield, $96 \%$ ee $)$ as a white solid. $[\alpha]_{\mathrm{D}}{ }^{25}-6.2\left(\mathrm{c} 0.74, \mathrm{CHCl}_{3}\right)$; m.p. 59.5-60.5 ${ }^{\circ} \mathrm{C} ;{ }^{1} \mathrm{H} \mathrm{NMR}\left(300 \mathrm{MHz}, \mathrm{CDCl}_{3}\right)$ $\delta 2.68-2.82(\mathrm{~m}, 2 \mathrm{H}), 3.69(\mathrm{~s}, 3 \mathrm{H}), 4.98(\mathrm{br}, 4 \mathrm{H}), 5.76(\mathrm{~s}, 1 \mathrm{H}), 5.91(\mathrm{~d}, J=$ $15.6 \mathrm{~Hz}, 1 \mathrm{H}), 6.51(\mathrm{~s}, 1 \mathrm{H}), 6.59-6.69(\mathrm{~m}, 1 \mathrm{H}), 7.23-7.40(\mathrm{~m}, 12 \mathrm{H}) ;{ }^{13} \mathrm{C} \mathrm{NMR}\left(125 \mathrm{MHz}, \mathrm{CDCl}_{3}\right) \delta 39.8$, 44.2, 51.7, 61.0, 67.5, 111.2, 115.8, 126.2, 126.9, 127.1, 127.7, 128.3, 128.4, 128.6, 128.9, 131.0, 132.2, 134.9, 135.4, 138.8, 141.5, 154.3, 165.7, 175.2; IR (ATR) 3318, 3032, 2950, 1709, 1608, 1483, 1257, 1176, 1041, 731, $696 \mathrm{~cm}^{-1}$; HRMS (ESI, positive) m/z for $\mathrm{C}_{28} \mathrm{H}_{25} \mathrm{BrN}_{2} \mathrm{NaO}_{5}[\mathrm{M}+\mathrm{Na}]^{+}$: calcd. 571.0839, found 571.0858; HPLC (DAICEL CHIRALPAK IF-3®, hexane: $i-\mathrm{PrOH}=70: 30,1.0 \mathrm{~mL} / \mathrm{min}, 254 \mathrm{~nm}$ ), $\mathrm{tR}=11.4 \mathrm{~min}$ (major), $23.4 \mathrm{~min}$ (minor).

\section{Methyl (R,E)-4-[1-benzyl-3-(benzyloxycarbonylamino)-6-bromo-2-oxoindolin-3-yl]but-2-enoate} (3f)

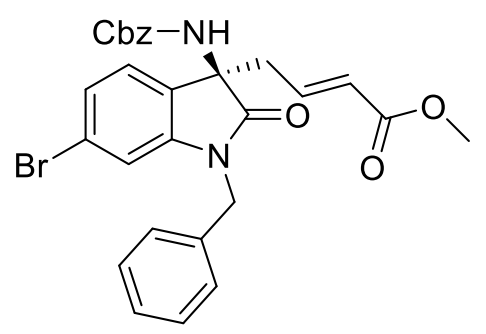

Reaction of ketimine $\mathbf{1 f}(0.10 \mathrm{mmol})$ and vinylketene silyl acetal 2a $(0.12$ $\mathrm{mmol})$ using $4 \mathbf{a}(0.01 \mathrm{mmol})$ and $\mathrm{Cu}(\mathrm{OTf})_{2}(0.01 \mathrm{mmol})$ in toluene $(1.0 \mathrm{~mL})$ at $-40{ }^{\circ} \mathrm{C}$ for $96 \mathrm{~h}$ gave $(R)-3 f(54.3 \mathrm{mg}, 99 \%$ yield, $98 \%$ ee) as a white solid. $[\alpha]_{\mathrm{D}}{ }^{25}-19.9$ (c 0.53, $\left.\mathrm{CHCl}_{3}\right)$; m.p. 58.2-59.0 ${ }^{\circ} \mathrm{C} ;{ }^{1} \mathrm{H}$ NMR $(300 \mathrm{MHz}$, $\left.\mathrm{CDCl}_{3}\right) \delta 2.67(\mathrm{dd}, J=7.8,14.0 \mathrm{~Hz}, 1 \mathrm{H}), 2.80(\mathrm{dd}, J=7.8,14.0 \mathrm{~Hz}, 1 \mathrm{H})$, $3.70(\mathrm{~s}, 3 \mathrm{H}), 4.97(\mathrm{br}, 4 \mathrm{H}), 5.74(\mathrm{~s}, 1 \mathrm{H}), 5.91(\mathrm{~d}, J=15.6 \mathrm{~Hz}, 1 \mathrm{H}), 6.63-6.73(\mathrm{~m}, 2 \mathrm{H}), 6.78(\mathrm{~s}, 1 \mathrm{H}), 7.10-$ 7.19 (m, 3H), 7.24-7.32 (m, 9H); $\left.{ }^{13} \mathrm{C} \mathrm{NMR} \mathrm{(125} \mathrm{MHz,} \mathrm{CDCl}_{3}\right) \delta$ 39.7, 44.2, 51.7, 60.8, 67.5, 113.0, 112.9, $124.3,125.9,126.9,127.1,127.7,127.9,128.3,128.4,128.4,128.5,128.9,134.9,139.0,143.7,154.4$, 165.7, 175.7; IR (ATR) 3237, 3032, 2951, 1710, 1605, 1486, 1254, 1177, 1028, 731, $696 \mathrm{~cm}^{-1}$; HRMS (ESI, positive) $\mathrm{m} / \mathrm{z}$ calcd for $\mathrm{C}_{28} \mathrm{H}_{25} \mathrm{BrN}_{2} \mathrm{NaO}_{5}[\mathrm{M}+\mathrm{Na}]^{+}$: calcd. 571.0839, found 571.0853; HPLC (DAICEL CHIRALPAK IF-3®, hexane: $i$-PrOH = 70:30, $1.0 \mathrm{~mL} / \mathrm{min}, 254 \mathrm{~nm}$ ), $\mathrm{tR}=12.8 \mathrm{~min}$ (major), $26.2 \mathrm{~min}$ (minor). 
Methyl (R,E)-4-[1-benzyl-3-(benzyloxycarbonylamino)-7-bromo-2-oxoindolin-3-yl]but-2-enoate (3g)

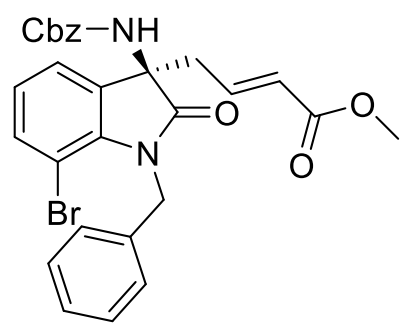

Reaction of ketimine $\mathbf{1 g}(0.10 \mathrm{mmol})$, and vinylketene silyl acetal $\mathbf{2 a}(0.12$ $\mathrm{mmol})$ using $4 \mathbf{a}(0.01 \mathrm{mmol})$ and $\mathrm{Cu}(\mathrm{OTf})_{2}(0.01 \mathrm{mmol})$ in toluene $(1.0 \mathrm{~mL})$ at $-40{ }^{\circ} \mathrm{C}$ for $72 \mathrm{~h}$ gave $(R)-3 \mathrm{~g}$ (54.4 mg, $99 \%$ yield, $97 \%$ ee) as a white solid. $[\alpha]_{\mathrm{D}}{ }^{25}-3.2$ (c 0.43, $\left.\mathrm{CHCl}_{3}\right)$; m.p. 57.2-58.0 ${ }^{\circ} \mathrm{C} ;{ }^{1} \mathrm{H} \mathrm{NMR}\left(300 \mathrm{MHz}, \mathrm{CDCl}_{3}\right) \delta$ 2.62-2.80 (m, 2H), $3.72(\mathrm{~s}, 3 \mathrm{H}), 4.97(\mathrm{~s}, 2 \mathrm{H}), 5.35(\mathrm{~s}, 2 \mathrm{H}), 5.72(\mathrm{~s}, 1 \mathrm{H}), 5.90(\mathrm{~d}$, $J=15.6 \mathrm{~Hz}, 1 \mathrm{H}), 6.67-6.77(\mathrm{~m}, 1 \mathrm{H}), 6.94(\mathrm{t}, J=7.8 \mathrm{~Hz}, 1 \mathrm{H}), 7.22-7.40(\mathrm{~m}, 12 \mathrm{H}) ;{ }^{13} \mathrm{C} \mathrm{NMR}(125 \mathrm{MHz}$, $\left.\mathrm{CDCl}_{3}\right) \delta 40.3,44.9,51.8,60.4,67.5,102.9,122.1,124.2,126.3,126.9,127.0,128.3,128.4,128.5,128.5$, 128.6, 132.3, 135.3, 137.4, 138.9, 140.0, 154.3, 165.7, 176.6; IR (ATR) 3324, 3033, 2950, 1710, 1608, 1450, 1255, 1165, 1027, 732, $695 \mathrm{~cm}^{-1}$; HRMS (ESI, positive) $\mathrm{m} / \mathrm{z}$ calcd for $\mathrm{C}_{28} \mathrm{H}_{25} \mathrm{BrN}_{2} \mathrm{NaO}_{5}[\mathrm{M}+\mathrm{Na}]^{+}$: calcd. 571.0839, found 571.0843; HPLC (DAICEL CHIRALPAK IF-3®, hexane:i-PrOH = 70:30, 1.0 $\mathrm{mL} / \mathrm{min}, 254 \mathrm{~nm}$ ), $\mathrm{tR}=13.2 \min$ (major), $35.2 \min$ (minor).

\section{Methyl (R,E)-4-[1-benzyl-3-(benzyloxycarbonylamino)-5-fluoro-2-oxoindolin-3-yl]but-2-enoate} (3h)

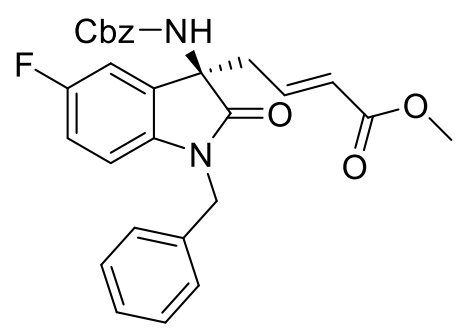

Reaction of ketimine $\mathbf{1 h}(0.10 \mathrm{mmol})$ and vinylketene silyl acetal $\mathbf{2 a}(0.12$ $\mathrm{mmol})$ using $4 \mathbf{a}(0.01 \mathrm{mmol})$ and $\mathrm{Cu}(\mathrm{OTf})_{2}(0.01 \mathrm{mmol})$ in toluene $(1.0 \mathrm{~mL})$ at $-40{ }^{\circ} \mathrm{C}$ for $168 \mathrm{~h}$ gave $(R)-3 \mathrm{~h}(48.4 \mathrm{mg}, 98 \%$ yield, $83 \%$ ee) as a white solid.

$[\alpha]_{\mathrm{D}}{ }^{25}-22.5$ (c $\left.0.54, \mathrm{CHCl}_{3}\right)$; m.p. 47.2-47.8 ${ }^{\circ} \mathrm{C} ;{ }^{1} \mathrm{H}$ NMR $(300 \mathrm{MHz}$, $\left.\mathrm{CDCl}_{3}\right) \delta 2.68-2.86(\mathrm{~m}, 2 \mathrm{H}), 3.70(\mathrm{~s}, 3 \mathrm{H}), 4.99(\mathrm{br}, 4 \mathrm{H}), 5.67(\mathrm{~s}, 1 \mathrm{H}), 5.92(\mathrm{~d}, J=15.6 \mathrm{~Hz}, 1 \mathrm{H}), 6.57$ (s, $1 \mathrm{H}), 6.62-6.73(\mathrm{~m}, 1 \mathrm{H}), 6.89(\mathrm{t}, J=8.7 \mathrm{~Hz}, 1 \mathrm{H}), 7.06(\mathrm{~d}, J=7.8 \mathrm{~Hz}, 1 \mathrm{H}), 7.29-7.33(\mathrm{~m}, 10 \mathrm{H}) ;{ }^{13} \mathrm{C} \mathrm{NMR}$ $\left(125 \mathrm{MHz} \mathrm{CDCl}_{3}\right) \delta 39.8,44.3,51.7,61.3,67.5,110.4\left(\mathrm{~d}, J_{\mathrm{C}-\mathrm{F}}=8.8 \mathrm{~Hz}\right), 111.3\left(\mathrm{~d}, J_{\mathrm{C}-\mathrm{F}}=25.0 \mathrm{~Hz}\right), 115.6$ $\left(\mathrm{d}, J_{\mathrm{C}-\mathrm{F}}=23.8 \mathrm{~Hz}\right), 126.8,127.1,127.7,128.3,128.4,128.5,128.9,130.6,135.1,135.5,138.3,138.9$, $154.4,159.3\left(\mathrm{~d}, J_{\mathrm{C}-\mathrm{F}}=239.8 \mathrm{~Hz}\right), 165.7,175.5 ;{ }^{19} \mathrm{~F} \mathrm{NMR}(282 \mathrm{MHz}) \delta-119.4$; IR (ATR) 3316, 3033, 2952, 1707, 1619, 1491, 1237, 1173, 1028, 732, $696 \mathrm{~cm}^{-1}$; HRMS (ESI, positive) m/z calcd for $\mathrm{C}_{28} \mathrm{H}_{25} \mathrm{FN}_{2} \mathrm{NaO}_{5}[\mathrm{M}+\mathrm{Na}]^{+}$: calcd. 511.1640, found 511.1636; HPLC (DAICEL CHIRALPAK IF-3®, hexane: $i-\mathrm{PrOH}=70: 30,1.0 \mathrm{~mL} / \mathrm{min}, 254 \mathrm{~nm}$ ), $\mathrm{tR}=10.7 \mathrm{~min}$ (major), $21.8 \mathrm{~min}$ (minor). 
Methyl (R,E)-4-[1-benzyl-3-(benzyloxycarbonylamino)-7-trifluoromethyl-2-oxoindolin-3-yl]but-2enoate (3i)

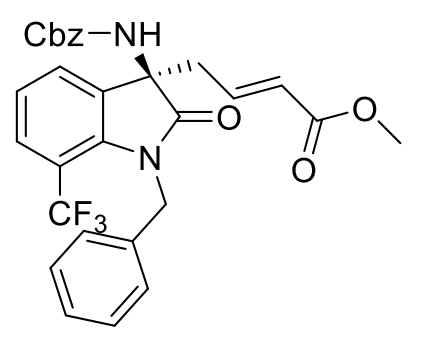

Reaction of ketimine $\mathbf{1 i}(0.10 \mathrm{mmol})$ and vinylketene silyl acetal $\mathbf{2 a}(0.12 \mathrm{mmol})$ using $4 \mathbf{a}(0.01 \mathrm{mmol})$ and $\mathrm{Cu}(\mathrm{OTf})_{2}(0.01 \mathrm{mmol})$ in toluene $(1.0 \mathrm{~mL})$ at $-40{ }^{\circ} \mathrm{C}$ for $168 \mathrm{~h}$ gave $(R)-3 \mathbf{i}(53.4 \mathrm{mg}, 99 \%$ yield, $97 \%$ ee) as a white solid. $[\alpha]_{\mathrm{D}}{ }^{25}+41.4\left(\mathrm{c} \mathrm{0.40}, \mathrm{CHCl}_{3}\right)$; m.p. $46.0-46.8^{\circ} \mathrm{C} ;{ }^{1} \mathrm{H}$ NMR $\left(300 \mathrm{MHz}, \mathrm{CDCl}_{3}\right)$ $\delta 2.62-2.79(\mathrm{~m}, 2 \mathrm{H}), 3.71(\mathrm{~s}, 3 \mathrm{H}), 4.97(\mathrm{~s}, 2 \mathrm{H}), 5.16(\mathrm{~s}, 2 \mathrm{H}), 5.75(\mathrm{~s}, 1 \mathrm{H}), 5.87$ $(\mathrm{d}, J=15.6 \mathrm{~Hz}, 1 \mathrm{H}), 6.66-6.77(\mathrm{~m}, 1 \mathrm{H}), 7.17-7.30(\mathrm{~m}, 11 \mathrm{H}), 7.47(\mathrm{~d}, J=7.5 \mathrm{~Hz}, 1 \mathrm{H}), 7.59$ (d, $J=8.1 \mathrm{~Hz}$, $1 \mathrm{H}) ;{ }^{13} \mathrm{C} \mathrm{NMR}\left(125 \mathrm{MHz}, \mathrm{CDCl}_{3}\right) \delta 40.2,46.3-46.4(\mathrm{~m}), 51.8,59.4,67.6,113.2\left(\mathrm{q}, J_{C-F}=33.3 \mathrm{~Hz}\right), 122.1$, $122.5,124.3,125.8,126.6,126.8,127.1,127.4-127.5(\mathrm{~m}), 128.3\left(\mathrm{~d}, J_{C-F}=3.6 \mathrm{~Hz}\right), 128.4,128.6,132.1$, 135.3, 136.2, 138.6, 140.7, 154.3, 165.6, 177.2; IR (ATR) 3325, 3034, 2953, 1715, 1597, 1453, 1258, 1120, 1028, 746, $695 \mathrm{~cm}^{-1}$; HRMS (ESI, positive) $\mathrm{m} / \mathrm{z}$ calcd for $\mathrm{C}_{29} \mathrm{H}_{25} \mathrm{~F}_{3} \mathrm{~N}_{2} \mathrm{NaO}_{5}[\mathrm{M}+\mathrm{Na}]^{+}$: calcd. 561.1608, found 561.1615; HPLC (DAICEL CHIRALPAK IF-3®, hexane: $i-\mathrm{PrOH}=70: 30,1.0 \mathrm{~mL} / \mathrm{min}$, $254 \mathrm{~nm}$ ), $\mathrm{tR}=11.2 \mathrm{~min}$ (major), $27.5 \mathrm{~min}$ (minor).

Methyl (R,E)-4-[1-benzyl-3-(benzyloxycarbonylamino)-5-chloro-2-oxoindolin-3-yl]but-2-enoate (3j)

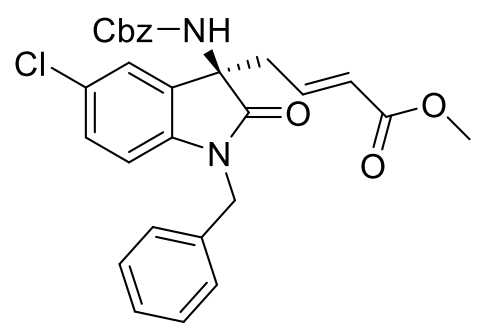

Reaction of ketimine $\mathbf{1 j}(0.10 \mathrm{mmol})$ and vinylketene silyl acetal $\mathbf{2 a}(0.12$ mmol) using $4 \mathbf{a}(0.02 \mathrm{mmol})$ and $\mathrm{Cu}(\mathrm{OTf})_{2}(0.02 \mathrm{mmol})$ in toluene $(1.0 \mathrm{~mL})$ at $-40{ }^{\circ} \mathrm{C}$ for $168 \mathrm{~h}$ gave $(R)-3 \mathbf{j}(46.9 \mathrm{mg}, 93 \%$ yield, $97 \%$ ee) as a white solid.

$[\alpha]_{\mathrm{D}}{ }^{25}-5.3$ (c 1.56, $\left.\mathrm{CHCl}_{3}\right)$; m.p. 61.2-62.0 ${ }^{\circ} \mathrm{C} ;{ }^{1} \mathrm{H} \mathrm{NMR}\left(300 \mathrm{MHz}, \mathrm{CDCl}_{3}\right)$ $\delta 2.68-2.83(\mathrm{~m}, 2 \mathrm{H}), 3.70(\mathrm{~s}, 3 \mathrm{H}), 4.98(\mathrm{br}, 4 \mathrm{H}), 5.67(\mathrm{~s}, 1 \mathrm{H}), 5.92(\mathrm{~d}, J=15.6 \mathrm{~Hz}, 1 \mathrm{H}), 6.57-6.70(\mathrm{~m}, 2 \mathrm{H})$, $7.15(\mathrm{dd}, J=2.1,8.4 \mathrm{~Hz}, 2 \mathrm{H}), 7.26-7.32(\mathrm{~m}, 10 \mathrm{H}) ;{ }^{13} \mathrm{C} \mathrm{NMR}\left(125 \mathrm{MHz}, \mathrm{CDCl}_{3}\right) \delta 39.8,44.2,51.7,61.1$, $67.5,110.7,123.5,126.9,127.1,127.7,128.3,128.4,128.5,128.5,128.9,129.2,130.7,135.0,135.4,138.8$, 141.0, 154.3, 165.7, 175.3; IR (ATR) 3310, 3033, 2951, 1715, 1611, 1487, 1257, 1176, 1042, 734, 696 $\mathrm{cm}^{-1}$; HRMS (ESI, positive) $\mathrm{m} / \mathrm{z}$ calcd for $\mathrm{C}_{28} \mathrm{H}_{25} \mathrm{ClN}_{2} \mathrm{NaO}_{5}[\mathrm{M}+\mathrm{Na}]^{+}$: calcd. 527.1344, found 527.1360; HPLC (DAICEL CHIRALPAK IF-3®, hexane: $i-\mathrm{PrOH}=70: 30,1.0 \mathrm{~mL} / \mathrm{min}, 254 \mathrm{~nm}$ ), tR = $23.4 \mathrm{~min}$ (major), $43.4 \mathrm{~min}$ (minor). 


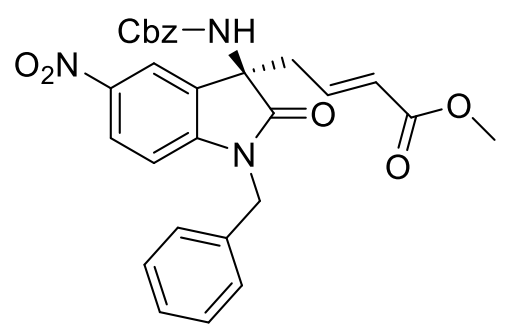

Reaction of ketimine $1 \mathbf{k}(0.10 \mathrm{mmol})$ and vinylketene silyl acetal $\mathbf{2 a}(0.12$ mmol) using $4 \mathbf{a}(0.02 \mathrm{mmol})$ and $\mathrm{Cu}(\mathrm{OTf})_{2}(0.02 \mathrm{mmol})$ in toluene $(1.0$ $\mathrm{mL})$ at $-40{ }^{\circ} \mathrm{C}$ for $192 \mathrm{~h}$ gave $(R)-3 \mathbf{k}(33.6 \mathrm{mg}, 65 \%$ yield, $79 \%$ ee) as a white solid.

$[\alpha]_{\mathrm{D}}{ }^{25}+8.0\left(\right.$ c $0.38, \mathrm{CHCl}_{3}$ ); m.p. 61.4-62.2 ${ }^{\circ} \mathrm{C}$; ${ }^{1} \mathrm{H} \mathrm{NMR}(300 \mathrm{MHz}$, $\left.\mathrm{CDCl}_{3}\right) \delta 2.74-2.86(\mathrm{~m}, 2 \mathrm{H}), 3.70(\mathrm{~s}, 3 \mathrm{H}), 5.00(\mathrm{br}, 4 \mathrm{H}), 5.79(\mathrm{~s}, 1 \mathrm{H}), 5.93(\mathrm{~d}, J=15.6 \mathrm{~Hz}, 1 \mathrm{H}), 6.58-6.72$ $(\mathrm{m}, 2 \mathrm{H}), 7.26-7.34(\mathrm{~m}, 10 \mathrm{H}), 8.14(\mathrm{~d}, J=10.8 \mathrm{~Hz}, 2 \mathrm{H}) ;{ }^{13} \mathrm{C} \mathrm{NMR}\left(125 \mathrm{MHz}, \mathrm{CDCl}_{3}\right) \delta 39.5,44.5,51.8$, $60.8,67.7,109.4,118.7,126.4,127.1,127.4,128.0,128.3,128.4,128.5,128.6,129.1,130.0,134.3,135.2$, 137.9, 143.6, 148.1 154.3, 165.5, 176.0; IR (ATR) 3328, 3033, 2952, 1714, 1614, 1334, 1258, 1175, 1043, 732, $696 \mathrm{~cm}^{-1}$; HRMS (ESI, positive) $\mathrm{m} / \mathrm{z}$ calcd for $\mathrm{C}_{28} \mathrm{H}_{25} \mathrm{~N}_{3} \mathrm{NaO}_{7}[\mathrm{M}+\mathrm{Na}]^{+}$: calcd. 538.1585, found 538.1578; HPLC (DAICEL CHIRALPAK IF-3®, hexane: $i$-PrOH = 70:30, $1.0 \mathrm{~mL} / \mathrm{min}, 254 \mathrm{~nm}$ ), tR = $24.0 \mathrm{~min}$ (minor), $34.8 \mathrm{~min}$ (major).

\section{Methyl (R,E)-4-[1-benzyl-3-(benzyloxycarbonylamino)-5-methyl-2-oxoindolin-3-yl]but-2-enoate} (31)

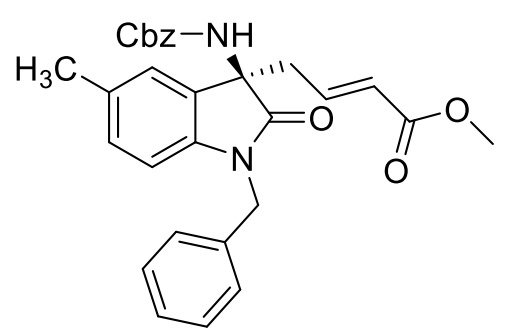

Reaction of ketimine 11 ( $0.10 \mathrm{mmol})$, and vinylketene silyl acetal $\mathbf{2 a}(0.12$ mmol) using $4 \mathbf{a}(0.01 \mathrm{mmol})$ and $\mathrm{Cu}(\mathrm{OTf})_{2}(0.01 \mathrm{mmol})$ in toluene $(1.0$ $\mathrm{mL})$ at $-40{ }^{\circ} \mathrm{C}$ for $168 \mathrm{~h}$ gave $(R)-31$ (48.0 $\mathrm{mg}, 99 \%$ yield, $92 \%$ ee) as a white solid.

$[\alpha]_{\mathrm{D}}{ }^{25}+25.7$ (c $\left.0.46, \mathrm{CHCl}_{3}\right)$; m.p. 50.8-51.4 ${ }^{\circ} \mathrm{C} ;{ }^{1} \mathrm{H}$ NMR $(300 \mathrm{MHz}$, $\left.\mathrm{CDCl}_{3}\right) \delta 2.30(\mathrm{~s}, 3 \mathrm{H}), 2.71(\mathrm{dd}, J=7.8,13.5 \mathrm{~Hz}, 1 \mathrm{H}), 2.82(\mathrm{dd}, J=7.8,13.5 \mathrm{~Hz}, 1 \mathrm{H}), 3.70(\mathrm{~s}, 3 \mathrm{H}), 4.98$ (br, 4H), $5.58(\mathrm{~s}, 1 \mathrm{H}), 5.93(\mathrm{~d}, J=15.6 \mathrm{~Hz}, 1 \mathrm{H}), 6.54(\mathrm{~d}, J=8.1 \mathrm{~Hz}, 1 \mathrm{H}), 6.66-6.77(\mathrm{~m}, 1 \mathrm{H}), 6.98(\mathrm{~d}, J=$ $7.8 \mathrm{~Hz}, 1 \mathrm{H}), 7.11(\mathrm{~s}, 1 \mathrm{H}), 6.96-7.29(\mathrm{~m}, 10 \mathrm{H}) ;{ }^{13} \mathrm{C} \mathrm{NMR}\left(125 \mathrm{MHz}, \mathrm{CD}_{3} \mathrm{OD}\right) \delta 19.8,39.2,43.6,50.7,61.6$, 66.4, 109.3, 122.9, 125.4, 127.0, 127.1, 127.6, 127.7, 128.1, 128.4, 129.1, 129.5, 132.8, 135.8, 136.4, 140.2, 140.3, 155.2, 166.1, 176.6; IR (ATR) 3322, 3032, 2950, 1705, 1604, 1496, 1235, 1179, 1044, 733, 696 $\mathrm{cm}^{-1}$; HRMS (ESI, positive) $\mathrm{m} / \mathrm{z}$ calcd for $\mathrm{C}_{29} \mathrm{H}_{28} \mathrm{~N}_{2} \mathrm{NaO}_{5}[\mathrm{M}+\mathrm{Na}]^{+}$: calcd. 507.1890, found 507.1894; HPLC (DAICEL CHIRALPAK IF-3®), hexane: $i$-PrOH = 70:30, $1.0 \mathrm{~mL} / \mathrm{min}, 254 \mathrm{~nm}$ ), tR = $13.4 \mathrm{~min}$ (major), $33.7 \mathrm{~min}$ (minor). 
Methyl (R,E)-4-[1-benzyl-3-(benzyloxycarbonylamino)-5-methoxy-2-oxoindolin-3-yl]but-2-enoate (3m)

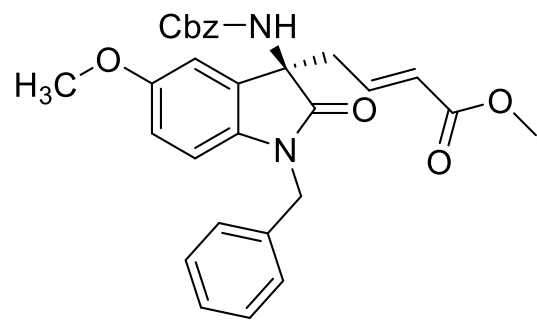

Reaction of $\mathbf{3 m}(0.01 \mathrm{mmol}), \mathrm{Cu}(\mathrm{OTf})_{2}(0.01 \mathrm{mmol})$, ketimine $\mathbf{1 m}(0.10$ $\mathrm{mmol})$, and vinylketene silyl acetal $2 \mathrm{a}(0.12 \mathrm{mmol})$ in toluene $(1.0 \mathrm{~mL})$ at $-40{ }^{\circ} \mathrm{C}$ for $120 \mathrm{~h}$ gave $(R)-3 \mathrm{~m}(49.4 \mathrm{mg}, 99 \%$ yield, $95 \%$ ee) as a white solid.

$[\alpha]_{\mathrm{D}}{ }^{25}-8.5$ (c $\left.0.43, \mathrm{CHCl}_{3}\right)$; m.p. 49.4-50.2 ${ }^{\circ} \mathrm{C} ;{ }^{1} \mathrm{H}$ NMR $(300 \mathrm{MHz}$, $\left.\mathrm{CDCl}_{3}\right) \delta 2.72(\mathrm{dd}, J=7.7,13.5 \mathrm{~Hz}, 1 \mathrm{H}), 2.82(\mathrm{dd}, J=7.7,13.5 \mathrm{~Hz}, 1 \mathrm{H}), 3.68(\mathrm{~s}, 3 \mathrm{H}), 3.74(\mathrm{~s}, 3 \mathrm{H}), 4.95$ (s, 2H), 4.98 (s, 2H), $5.67(\mathrm{~s}, 1 \mathrm{H}), 5.91(\mathrm{~d}, J=15.7 \mathrm{~Hz}, 1 \mathrm{H}), 6.54(\mathrm{~d}, J=8.4 \mathrm{~Hz}, 1 \mathrm{H}), 6.64-6.74(\mathrm{~m}, 2 \mathrm{H})$, $6.92(\mathrm{~s}, 1 \mathrm{H}), 7.23-7.34(\mathrm{~m}, 10 \mathrm{H}) ;{ }^{13} \mathrm{C} \mathrm{NMR}\left(125 \mathrm{MHz}, \mathrm{CDCl}_{3}\right) \delta$ 40.0, 44.2, 51.7, 55.7, 61.4, 67.4, 110.2, $110.5,113.5,126.5,127.1,127.5,128.3,128.3,128.5,128.8,130.2$, 135.5, 135.6, 135.7, 139.5, 154.4, 156.1, 165.8, 175.4; IR (ATR) 3319, 3033, 2950, 1704, 1604, 1495, 1240, 1177, 1027, 733, $696 \mathrm{~cm}^{-1}$; HRMS (ESI, positive) $\mathrm{m} / \mathrm{z}$ calcd. for $\mathrm{C}_{29} \mathrm{H}_{28} \mathrm{~N}_{2} \mathrm{NaO}_{6}[\mathrm{M}+\mathrm{Na}]^{+}$: calc. 523.1840, found 523.1843; HPLC (DAICEL CHIRALPAK IF-3®, hexane: $i$-PrOH = 70:30, $1.0 \mathrm{~mL} / \mathrm{min}, 254 \mathrm{~nm}$ ), tR = 18.9 min (major), $42.0 \mathrm{~min}$ (minor).

\section{Methyl}

(R,E)-4-[1-benzyl-3-(benzyloxycarbonylamino)-5,7-dimethyl-2-oxoindolin-3-yl]but-2enoate (3n)

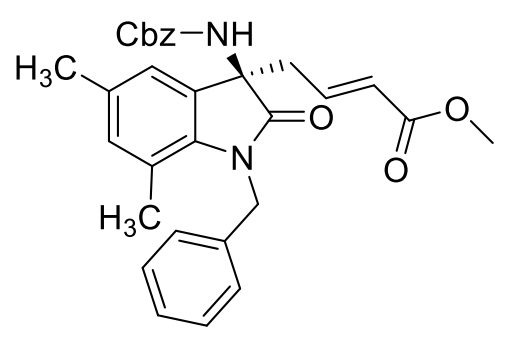

Reaction of ketimine $1 \mathbf{n}(0.10 \mathrm{mmol})$, and vinylketene silyl acetal 2a $(0.12$ mmol) using $4 \mathbf{a}(0.01 \mathrm{mmol})$ and $\mathrm{Cu}(\mathrm{OTf})_{2}(0.01 \mathrm{mmol})$ in toluene $(1.0$ $\mathrm{mL})$ at $-40{ }^{\circ} \mathrm{C}$ for $144 \mathrm{~h}$ gave $(R)-3 n(49.3 \mathrm{mg}, 99 \%$ yield, $93 \%$ ee) as a white solid.

$[\alpha]_{\mathrm{D}}{ }^{25}+27.9\left(\mathrm{c} 0.43, \mathrm{CHCl}_{3}\right)$; m.p. 58.0-59.0 ${ }^{\circ} \mathrm{C} ;{ }^{1} \mathrm{H}$ NMR $(300 \mathrm{MHz}$, $\left.\mathrm{CDCl}_{3}\right) \delta 2.14(\mathrm{~s}, 3 \mathrm{H}), 2.27(\mathrm{~s}, 3 \mathrm{H}), 2.67-2.82(\mathrm{~m}, 2 \mathrm{H}), 3.71(\mathrm{~s}, 3 \mathrm{H}), 4.92-5.03(\mathrm{~m}, 2 \mathrm{H}), 5.13(\mathrm{~s}, 2 \mathrm{H}), 5.63$ (s, 1H), $5.92(\mathrm{~d}, J=15.6 \mathrm{~Hz}, 1 \mathrm{H}), 6.70-6.78(\mathrm{~m}, 2 \mathrm{H}), 6.97(\mathrm{~s}, 1 \mathrm{H}), 7.21-7.31(\mathrm{~m}, 10 \mathrm{H}) ;{ }^{13} \mathrm{C}$ NMR $(125$ $\left.\mathrm{MHz}, \mathrm{CDCl}_{3}\right) \delta 18.5,20.8,40.6$ 45.4, 51.7, 60.5, 67.3, 119.9, 121.6, 125.7, 126.4, 127.0, 128.2, 128.3, 128.5, 128.8, 129.8, 132.5, 133.9, 135.6, 137.6, 138.0, 139.9, 154.3, 165.9, 176.6; IR (ATR) 3314, 3032, 2951, 1704, 1604, 1482, 1250, 1174, 1026, 730, $696 \mathrm{~cm}^{-1}$; HRMS (ESI, positive) m/z for $\mathrm{C}_{30} \mathrm{H}_{30} \mathrm{~N}_{2} \mathrm{NaO}_{5}$ $[\mathrm{M}+\mathrm{Na}]^{+}$: calcd. 521.2047, found 521.2071; HPLC (DAICEL CHIRALPAK IF-3®, hexane: $i-\operatorname{PrOH}=$ 70:30, $1.0 \mathrm{~mL} / \mathrm{min}, 254 \mathrm{~nm}$ ), $\mathrm{tR}=13.8 \mathrm{~min}$ (major), $35.9 \mathrm{~min}$ (minor). 


\section{b]pyridin-3-yl]but-2-enoate (3o)}

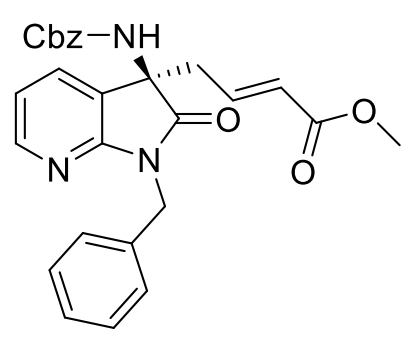

Reaction of ketimine $10(0.10 \mathrm{mmol})$, and vinylketene silyl acetal 2a $(0.12$ $\mathrm{mmol})$ using $4 \mathbf{a}(0.011 \mathrm{mmol})$ and $\mathrm{Cu}(\mathrm{OTf})_{2}(0.01 \mathrm{mmol})$ in toluene $(1.0 \mathrm{~mL})$ at $-40{ }^{\circ} \mathrm{C}$ for $72 \mathrm{~h}$ gave $(R)-30$ (47.1 $\mathrm{mg}, 99 \%$ yield, $96 \%$ ee) as a white solid. $[\alpha]_{\mathrm{D}}{ }^{25}-14.2\left(\mathrm{c} 0.97, \mathrm{CHCl}_{3}\right) ;$ m.p. $41.8-42.4{ }^{\circ} \mathrm{C} ;{ }^{1} \mathrm{H}$ NMR $\left(300 \mathrm{MHz}, \mathrm{CDCl}_{3}\right) \delta$ $2.63(\mathrm{dd}, J=7.8,14.0 \mathrm{~Hz}, 1 \mathrm{H}), 2.82(\mathrm{dd}, J=7.8,14.0 \mathrm{~Hz}, 1 \mathrm{H}), 3.67$ (s, 3H), $4.96(\mathrm{br}, 4 \mathrm{H}), 5.75(\mathrm{~s}, 1 \mathrm{H}), 5.86(\mathrm{~d}, J=15.3 \mathrm{~Hz}, 1 \mathrm{H}) 6.61-6.72(\mathrm{~m}, 1 \mathrm{H}), 6.95(\mathrm{t}, J=6.3 \mathrm{~Hz}, 1 \mathrm{H}), 7.20$ $7.35(\mathrm{~m}, 8 \mathrm{H}), 7.42(\mathrm{~d}, J=6.3 \mathrm{~Hz}, 2 \mathrm{H}), 7.54(\mathrm{~s}, 1 \mathrm{H}), 8.21(\mathrm{~d}, J=5.4 \mathrm{~Hz}, 1 \mathrm{H}) ;{ }^{13} \mathrm{C} \mathrm{NMR}\left(125 \mathrm{MHz}, \mathrm{CDCl}_{3}\right)$ $\delta 39.2,42.9,51.7,60.6,67.4,118.6,123.4,126.9,127.4,128.1,128.1,128.2,128.3,128.5,131.1,135.4$, 136.3, 138.8, 148.2, 154.4, 156.0, 165.6, 175.4; IR (ATR) 3328, 3032, 2951, 1713, 1597, 1450, 1253, 1178, 1027, 738, $696 \mathrm{~cm}^{-1}$; HRMS (ESI, positive) $\mathrm{m} / \mathrm{z}$ for $\mathrm{C}_{27} \mathrm{H}_{25} \mathrm{~N}_{3} \mathrm{NaO}_{5}[\mathrm{M}+\mathrm{Na}]^{+}$: calcd. 494.1686, found 494.1688; HPLC (DAICEL CHIRALPAK IF-3®, hexane: $i$-PrOH = 70:30, $1.0 \mathrm{~mL} / \mathrm{min}, 254 \mathrm{~nm}$ ), $\mathrm{tR}=11.2 \min$ (major), $28.6 \min$ (minor).

Methyl (R,E)-4-[1-benzyl-3-(benzyloxycarbonylamino)-2-oxoindolin-3-yl]-2-methylbut-2-enoate (3p)

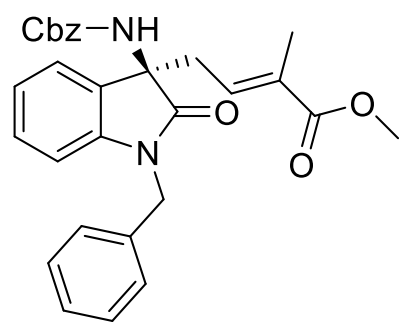

Reaction of ketimine $\mathbf{1 d}(0.10 \mathrm{mmol})$ and vinylketene silyl acetal $\mathbf{2 b}(0.30$ mmol) using $4 \mathbf{a}(0.011 \mathrm{mmol})$ and $\mathrm{Cu}(\mathrm{OTf})_{2}(0.01 \mathrm{mmol})$ in toluene $(1.0 \mathrm{~mL})$ at $0{ }^{\circ} \mathrm{C}$ for $66 \mathrm{~h}$ gave $(R)-3 p$ (42.2 mg, $87 \%$ yield, $87 \%$ ee) as a white solid.

$[\alpha]_{\mathrm{D}}{ }^{25}-8.1\left(\mathrm{c} 0.56, \mathrm{CHCl}_{3}\right)$; m.p. 37.8-38.6 ${ }^{\circ} \mathrm{C} ;{ }^{1} \mathrm{H} \mathrm{NMR}\left(300 \mathrm{MHz}, \mathrm{CDCl}_{3}\right) \delta$ $1.76(\mathrm{~s}, 3 \mathrm{H}), 2.71-2.88(\mathrm{~m}, 2 \mathrm{H}), 3.68(\mathrm{~s}, 3 \mathrm{H}), 4.97$ (br, 4H), $5.64(\mathrm{~s}, 1 \mathrm{H}), 6.56-$ $6.64(\mathrm{~m}, 2 \mathrm{H}), 7.04$ (t, $J=7.5 \mathrm{~Hz}, 1 \mathrm{H}), 7.16-7.30(\mathrm{~m}, 12 \mathrm{H}) ;{ }^{13} \mathrm{C} \mathrm{NMR}\left(125 \mathrm{MHz}, \mathrm{CDCl}_{3}\right) \delta 12.8,36.5,44.1$, 52.0, 61.1, 67.3, 109.6, 122.9, 123.0, 127.1, 127.5, 128.2, 128.4, 128.5, 128.7, 129.2, 132.2, 132.6, 135.5, 142.4, 154.4, 167.6, 176.0; IR (ATR) 3326, 3033, 2952, 1706, 1614, 1489, 1257, 1085, 1026, 735, 696 $\mathrm{cm}^{-1}$; HRMS (ESI, positive) $\mathrm{m} / \mathrm{z}$ for $\mathrm{C}_{29} \mathrm{H}_{28} \mathrm{~N}_{2} \mathrm{NaO}_{5}[\mathrm{M}+\mathrm{Na}]^{+}$: calcd. 507.1890, found 507.1894; HPLC (DAICEL CHIRALPAK IF-3®, hexane: $i-\mathrm{PrOH}=70: 30,1.0 \mathrm{~mL} / \mathrm{min}, 254 \mathrm{~nm}$ ), tR = 11.9 min (major), $27.8 \min$ (minor). 
yl]carbamate (3q)

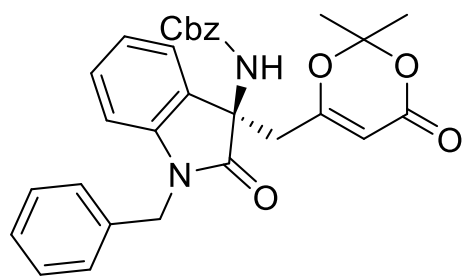

Reaction of ketimine $1 \mathbf{d}(0.10 \mathrm{mmol})$ and vinylketene silyl acetal $2 \mathbf{c}(0.12$ $\mathrm{mmol})$ using $4 \mathbf{a}(0.022 \mathrm{mmol})$ and $\mathrm{Cu}(\mathrm{OTf})_{2}(0.02 \mathrm{mmol})$ in toluene $(1.0$ $\mathrm{mL})$ at r.t. for $168 \mathrm{~h}$ gave $(R)-3 q(34.0 \mathrm{mg}, 66 \%$ yield, $81 \%$ ee) as a white solid.

$[\alpha]_{\mathrm{D}}{ }^{25}-17.7\left(\mathrm{c} 0.52, \mathrm{CHCl}_{3}\right)$; m.p. 53.2-53.8 ${ }^{\circ} \mathrm{C} ;{ }^{1} \mathrm{H} \mathrm{NMR}\left(300 \mathrm{MHz}, \mathrm{CDCl}_{3}\right) \delta 1.32(\mathrm{~s}, 3 \mathrm{H}), 1.49(\mathrm{~s}, 3 \mathrm{H})$, $2.86(\mathrm{~s}, 2 \mathrm{H}), 4.87-5.05(\mathrm{~m}, 4 \mathrm{H}), 5.17(\mathrm{~s}, 1 \mathrm{H}), 5.86(\mathrm{~s}, 1 \mathrm{H}), 6.71(\mathrm{~d}, J=8.1 \mathrm{~Hz}, 1 \mathrm{H}), 7.04(\mathrm{t}, J=7.5 \mathrm{~Hz}$, $1 \mathrm{H}), 7.18-7.36(\mathrm{~m}, 12 \mathrm{H}) ;{ }^{13} \mathrm{C} \mathrm{NMR}\left(125 \mathrm{MHz}, \mathrm{CDCl}_{3}\right) \delta 24.6,24.7,40.3,44.3,60.6,67.3,97.1,107.0$, 109.6, 123.0, 124.2, 127.5, 127.8, 128.1, 128.3, 128.5, 128.9, 129.6, 135.2, 135.6, 142.6, 154.3, 160.1, 164.4, 174.9; IR (ATR) 3309, 3033, 2944, 1710, 1614, 1376, 1254, 1202, 1010, 750, $696 \mathrm{~cm}^{-1}$; HRMS (ESI, positive) $\mathrm{m} / \mathrm{z}$ for $\mathrm{C}_{30} \mathrm{H}_{28} \mathrm{~N}_{2} \mathrm{NaO}_{6}[\mathrm{M}+\mathrm{Na}]^{+}$: calcd. 535.1840, found 535.1851; HPLC (DAICEL CHIRALPAK IF-3®, hexane: $i-\mathrm{PrOH}=70: 30,1.0 \mathrm{~mL} / \mathrm{min}, 254 \mathrm{~nm}$ ), tR = $11.1 \mathrm{~min}$ (major), $27.1 \mathrm{~min}$ (minor).

\section{Methyl (E)-4-[(R)-1-benzyl-3-(benzyloxycarbonylamino)-2-oxoindolin-3-yl]-pent-2-enoate (3r)}

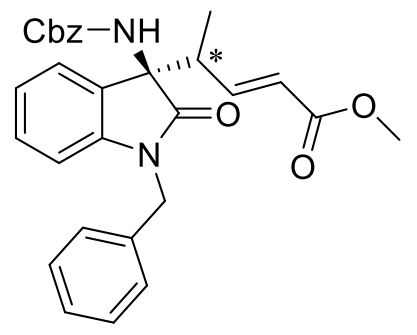

Reaction of ketimine $\mathbf{1 d}(0.10 \mathrm{mmol})$ and vinylketene silyl acetal $\mathbf{2 d}(0.30$ $\mathrm{mmol})$ using $4 \mathbf{a}(0.011 \mathrm{mmol})$ and $\mathrm{Cu}(\mathrm{OTf})_{2}(0.01 \mathrm{mmol})$ in toluene $(1.0 \mathrm{~mL})$ at $-40{ }^{\circ} \mathrm{C}$ for $96 \mathrm{~h}$ gave $(R)-3 \mathbf{r}(48.5 \mathrm{mg}, 99 \%$ yield, $\mathrm{dr}=60: 40$; major: $93 \%$ ee, minor: $88 \%$ ee) as a white solid.

$[\alpha]_{\mathrm{D}}{ }^{25}-15.5$ (c 0.66, $\left.\mathrm{CHCl}_{3}\right)$; m.p. 47.0-48.0 ${ }^{\circ} \mathrm{C} ;{ }^{1} \mathrm{H} \mathrm{NMR}\left(300 \mathrm{MHz}, \mathrm{CDCl}_{3}\right)$ $\delta 0.77$ (d, $J=6.9 \mathrm{~Hz}, 3 \mathrm{H}$, major), 1.00 (d, $J=6.9 \mathrm{~Hz}, 3 \mathrm{H}$, minor), 2.87-3.02 (m, 2H) 3.71 (s, 3H, minor), 3.77 (s, 3H, major), 4.96 (br, 4H), 5.44 (s, 1H), 5.52 (s, 1H), 5.93 (d, $J=15.6 \mathrm{~Hz}, 1 \mathrm{H}$, minor), 6.04 (d, $J$ $=15.6 \mathrm{~Hz}, 1 \mathrm{H}$, major), 6.64-6.72 (m, 2H), 6.87-6.99 (m, 2H), 7.03-7.08 (m, 2H), 7.18-7.34 (m, 11H); ${ }^{13} \mathrm{C}$ NMR (125 MHz, $\mathrm{CDCl}_{3}$ ) $\delta 13.6$ (major), 14.1 (minor), 43.4 (major), 43.9 (minor), 44.2 (major), 44.4 (minor), 51.7 (minor), 51.9 (major), 63.7 (major), 63.8 (minor), 67.3 (minor), 67.4 (major), 109.4, 109.5, $122.9,123.2$, 123.9, 124.2, 124.6, 127.3, 127.4, 127.5, 127.6, 128.1, 128.3, 128.3, 128.4, 128.5, 128.5, 128.7 (minor), 128.8 (major), 129.3 (minor), 129.4 (major), 135.6, 143.3 (minor), 143.5 (major), 145.9 (minor), 145.9 (major), 154.1 (major), 154.4 (minor), 166.0, 175.3 (minor), 175.6 (major); IR (ATR) 3328, 3033, 2951, 1706, 1613, 1488, 1255, 1179, 1003, 729, $696 \mathrm{~cm}^{-1}$; HRMS (ESI, positive) m/z for $\mathrm{C}_{29} \mathrm{H}_{28} \mathrm{~N}_{2} \mathrm{NaO}_{5}[\mathrm{M}+\mathrm{Na}]^{+}$: calcd. 507.1890, found 507.1910; HPLC (DAICEL CHIRALPAK IF-3®, hexane: $i-\mathrm{PrOH}=80: 20,1.0 \mathrm{~mL} / \mathrm{min}, 254 \mathrm{~nm}$ ), major: $\mathrm{tR}=21.3 \mathrm{~min}$ (major), $56.7 \mathrm{~min}$ (minor), minor: $\mathrm{tR}$ $=25.0 \mathrm{~min}$ (major), $46.5 \mathrm{~min}$ (minor). 

methyl (3s)

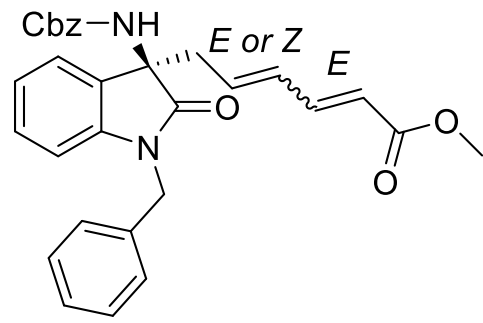

Reaction of ketimine $\mathbf{1 d}(0.10 \mathrm{mmol})$ and vinylketene silyl acetal $\mathbf{2 e}$ $(0.12 \mathrm{mmol})$ using $4 \mathbf{a}(0.011 \mathrm{mmol})$ and $\mathrm{Cu}(\mathrm{OTf})_{2}(0.01 \mathrm{mmol})$ in toluene $(1.0 \mathrm{~mL})$ at $-40{ }^{\circ} \mathrm{C}$ for $168 \mathrm{~h}$ gave $(R)-3 \mathrm{~s}(40.3 \mathrm{mg}, 81 \%$ yield, $\mathrm{E} / \mathrm{Z}$ mixture, major:minor $=85: 15)$ as a white solid. Ee was determined after transformation to $\mathbf{5}$.

$[\alpha]_{\mathrm{D}}{ }^{25}-85.8$ (c 0.27, $\left.\mathrm{CHCl}_{3}\right)$; m.p. 52.4-53.2 ${ }^{\circ} \mathrm{C} ;{ }^{1} \mathrm{H}$ NMR $\left(300 \mathrm{MHz}, \mathrm{CD}_{3} \mathrm{CN}\right) \delta 2.73(\mathrm{dd}, J=7.5,13.2$ $\mathrm{Hz}, 1 \mathrm{H}$, major), 2.82 (dd, $J=7.5,13.2 \mathrm{~Hz}, 1 \mathrm{H}$, major), 2.89-3.04 (m, 2H, minor) 3.70 (s, 3H, minor), 3.70 (s, 3H, major), 4.88-4.98 (m, 4H), 5.46 (dd, $J=8.7,17.4 \mathrm{~Hz}, 1 \mathrm{H}$, minor), 5.68-5.76 (m, 1H, major), 5.81 (d, $J=15.6 \mathrm{~Hz}, 1 \mathrm{H}$, major), 5.89 (d, $J=15.6 \mathrm{~Hz}, 1 \mathrm{H}$, minor), 6.11-6.24 (m, 1H), 6.63 (s, 1H), $6.72(\mathrm{~d}, J$ $=7.8 \mathrm{~Hz}, 1 \mathrm{H}), 6.70-7.09(\mathrm{~m}, 2 \mathrm{H}), 7.21-7.24(\mathrm{t}, J=7.8 \mathrm{~Hz}, 1 \mathrm{H}), 7.30-7.46(\mathrm{~m}, 10 \mathrm{H}) ;{ }^{13} \mathrm{C} \mathrm{NMR}(125 \mathrm{MHz}$, $\left.\mathrm{CDCl}_{3}\right) \delta 35.9$ (minor), 40.9 (major), 44.1, 51.6 (major), 51.7 (minor), 61.1 (minor), 61.4 (major), 67.3, 109.4 (minor), 109.6 (major), 121.4, 122.9 (major), 123.0 (minor), 123.2, 127.2 (major), 127.2 (minor), 127.3, 127.6, 128.2, 128.5, 128.7, 128.8, 129.1 (minor) 129.2 (major), 130.5, 131.2, 133.7 (major), 133.7 (minor), 135.5 (major), 135.6 (minor), 137.9, 142.4 (minor), 143.4, 154.4 (major), 154.4 (minor), 167.1 (minor), 167.2 (major), 175.8 (minor), 175.9 (major); IR (ATR) 3318, 3032, 2950, 1705, 1614, 1488, 1256, 1177, 1001, 732, $695 \mathrm{~cm}^{-1}$; HRMS (ESI, positive) $\mathrm{m} / \mathrm{z}$ for $\mathrm{C}_{30} \mathrm{H}_{28} \mathrm{~N}_{2} \mathrm{NaO}_{5}[\mathrm{M}+\mathrm{Na}]^{+}$: calcd. 519.1890, found 519.1887

\section{Methyl (R)-6-(3-amino-1-benzyl-2-oxoindolin-3-yl)hexanoate (5)}

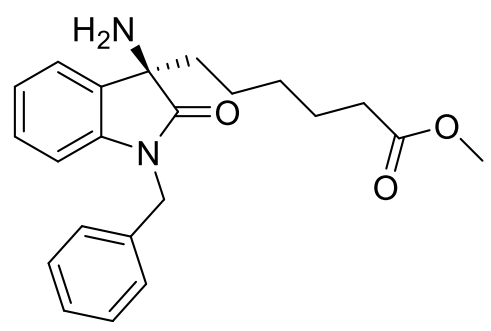

$\mathrm{Pd} / \mathrm{C}(20 \mathrm{wt} \%$ on carbon, $8.1 \mathrm{mg})$ was added to a solution of $3 \mathrm{~s}(40.3 \mathrm{mg}$, $0.081 \mathrm{mmol})$ in methanol $(0.8 \mathrm{~mL})$. The reaction mixture was degassed in vacuo, placed under $\mathrm{H}_{2}$ (balloon), stirred at r.t. for $3 \mathrm{~h}$. The mixture was filtered and washed with methanol, then the solvent was removed under reduced pressure to give the crude product, which was purified by silica gel column chromatography (ethyl acetate) to afford 5 (27.8 $\mathrm{mg}, 94 \%$ yield, $89 \%$ ee) as a colorless oil. $[\alpha]_{\mathrm{D}}{ }^{25}+23.0\left(\mathrm{c} 0.84, \mathrm{CHCl}_{3}\right) ;{ }^{1} \mathrm{H}$ NMR $\left(300 \mathrm{MHz}, \mathrm{CDCl}_{3}\right) \delta$ 0.93-1.02 (m, 1H), 1.06-1.16 (m, 1H), 1.19$1.28(\mathrm{~m}, 2 \mathrm{H}), 1.46-1.56(\mathrm{~m}, 2 \mathrm{H}), 1.79(\mathrm{~s}, 2 \mathrm{H}), 1.88(\mathrm{t}, J=7.2 \mathrm{~Hz}, 2 \mathrm{H}), 2.20(\mathrm{t}, J=7.5 \mathrm{~Hz}, 1 \mathrm{H}), 3.63(\mathrm{~s}$, $3 \mathrm{H}), 4.77(\mathrm{~d}, J=15.8 \mathrm{~Hz}, 1 \mathrm{H}), 5.01(\mathrm{~d}, J=15.8 \mathrm{~Hz}, 1 \mathrm{H}), 6.75(\mathrm{~d}, J=7.8 \mathrm{~Hz}, 1 \mathrm{H}), 7.06(\mathrm{t}, J=7.5 \mathrm{~Hz}, 1 \mathrm{H})$, $7.20(\mathrm{t}, J=7.8 \mathrm{~Hz}, 1 \mathrm{H}), 7.29-7.37(\mathrm{~m}, 6 \mathrm{H}) ;{ }^{13} \mathrm{C} \mathrm{NMR}\left(125 \mathrm{MHz}, \mathrm{CDCl}_{3}\right) \delta 23.4,24.6,33.8,39.2,43.8$, 51.5, 61.1, 109.2, 122.9, 123.7, 127.4, 127.7, 128.8, 131.7, 135.9, 142.5, 174.1, 180.8; IR (ATR) 3361, 3032, 2935, 2858, 1710, 1613, 1466, 1348, 1172, 750, $697 \mathrm{~cm}^{-1}$; HRMS (ESI, positive) m/z for $\mathrm{C}_{22} \mathrm{H}_{26} \mathrm{~N}_{2} \mathrm{NaO}_{3}[\mathrm{M}+\mathrm{Na}]^{+}$: calcd. 389.1836, found 389.1837; HPLC (for its $N$ - $p$-methoxybenzenesulfonyl 
protected derivative, DAICEL CHIRALPAK IB N-3®, hexane: $i$-PrOH $=70: 30,1.0 \mathrm{~mL} / \mathrm{min}, 254 \mathrm{~nm})$, tR $=18.6 \min$ (major), $35.4 \min$ (minor).

\section{Methyl (R)-4-(3-amino-1-benzyl-2-oxoindolin-3-yl)butanoate (6)}

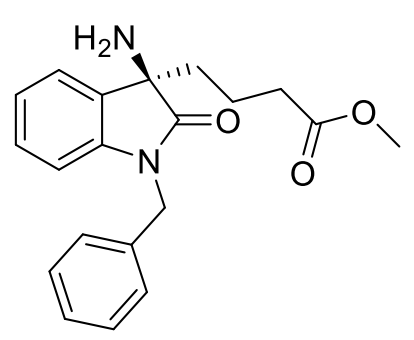

$\mathrm{Pd} / \mathrm{C}(20 \mathrm{wt} \%$ on carbon, $4.7 \mathrm{mg})$ was added to a solution of $\mathbf{3 d}(23.5 \mathrm{mg}, 0.05$ $\mathrm{mmol})$ in methanol $(0.5 \mathrm{~mL})$. The reaction mixture was degassed in vacuo, placed under $\mathrm{H}_{2}$ (balloon), stirred at r.t. for $6 \mathrm{~h}$. The mixture was filtered and washed with methanol, then the solvent was removed under reduced pressure to give the crude product, which was purified by silica gel column chromatography

(ethyl acetate) to afford $\mathbf{6}(13.5 \mathrm{mg}, 80 \%$ yield) as a colorless oil. Ee was determined after transformation to 6 .

$[\alpha]_{\mathrm{D}}{ }^{25}-3.3\left(\mathrm{c} 0.32, \mathrm{CHCl}_{3}\right) ;{ }^{1} \mathrm{H}$ NMR $\left(300 \mathrm{MHz}, \mathrm{CDCl}_{3}\right) \delta 1.34-1.52$ (m, 2H), 1.73 (br, 2H), 1.84-1.97 $(\mathrm{m}, 2 \mathrm{H}), 2.18-2.27(\mathrm{~m}, 2 \mathrm{H}), 3.62(\mathrm{~s}, 3 \mathrm{H}), 4.81(\mathrm{~d}, J=15.6 \mathrm{~Hz}, 1 \mathrm{H}), 4.99$ (d, $J=15.6 \mathrm{~Hz}, 1 \mathrm{H}), 6.74(\mathrm{~d}, J=$ $7.8 \mathrm{~Hz}, 1 \mathrm{H}), 7.06(\mathrm{t}, J=7.5 \mathrm{~Hz}, 1 \mathrm{H}), 7.20(\mathrm{t}, J=7.8 \mathrm{~Hz}, 1 \mathrm{H}), 7.29-7.34(\mathrm{~m}, 6 \mathrm{H}) ;{ }^{13} \mathrm{C} \mathrm{NMR}(125 \mathrm{MHz}$, $\left.\mathrm{CD}_{3} \mathrm{OD}\right) \delta 19.1,33.0,38.0,43.1,50.5,60.6,109.3,122.8,123.2,126.9,127.0,127.2,128.3,128.4,128.6$, 131.1, 135.8, 142.4, 173.5, 180.0; IR (ATR) 3031, 2950, 1709, 1612, 1466, 1348, 1254, 1173, 749, 697, $630 \mathrm{~cm}^{-1}$; HRMS (ESI, positive) $\mathrm{m} / \mathrm{z}$ for $\mathrm{C}_{20} \mathrm{H}_{22} \mathrm{~N}_{2} \mathrm{NaO}_{3}[\mathrm{M}+\mathrm{Na}]^{+}$: calcd. 361.1523, found 361.1516.

\section{(R)-1-Benzylspiro[indoline-3,2'-piperidine]-2,6'-dione (7)}

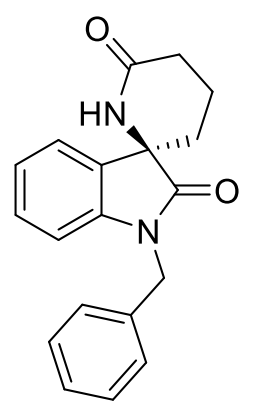

To a solution of $5(13.5 \mathrm{mg}, 0.04 \mathrm{mmol})$ in dry toluene $(0.4 \mathrm{~mL})$, trifluoroacetic acid $(6.1$ $\mu \mathrm{L}, 0.08 \mathrm{mmol}$ ) was added at r.t. under nitrogen atmosphere. The reaction mixture was heated at $90{ }^{\circ} \mathrm{C}$ with oil bath for $6 \mathrm{~h}$, and allowed to cool down to r.t., then quenched with sat. $\mathrm{NaHCO}_{3}$, and aqueous layer was extracted with ethyl acetate. The combined organic layer was dried over $\mathrm{Na}_{2} \mathrm{SO}_{4}$ and concentrated under reduced pressure to give the crude product, which was purified by silica gel column chromatography (ethyl acetate) to afford 7 (12.0 mg, 97\% yield, 97\% ee) as a white solid.

$[\alpha]_{\mathrm{D}}{ }^{25}+110.9\left(\mathrm{c} 0.25, \mathrm{CHCl}_{3}\right)$; m.p. 170.4-171.0 ${ }^{\circ} \mathrm{C} ;{ }^{1} \mathrm{H} \mathrm{NMR}\left(300 \mathrm{MHz}, \mathrm{CDCl}_{3}\right) \delta 1.90-2.12(\mathrm{~m}, 3 \mathrm{H})$, 2.47-2.69 (m, 3H), 4.79 (d, $J=15.6 \mathrm{~Hz}, 1 \mathrm{H}), 4.98(\mathrm{~d}, J=15.6 \mathrm{~Hz}, 1 \mathrm{H}), 5.76(\mathrm{~s}, 1 \mathrm{H}), 6.75$ (d, $J=7.8 \mathrm{~Hz}$, $1 \mathrm{H}), 7.07(\mathrm{t}, J=7.5 \mathrm{~Hz}, 1 \mathrm{H}), 7.21-7.36(\mathrm{~m}, 7 \mathrm{H}) ;{ }^{13} \mathrm{C} \mathrm{NMR}\left(125 \mathrm{MHz}, \mathrm{CDCl}_{3}\right) \delta 16.4,30.9,32.6,43.9$, 61.6, 109.7, 123.3, 123.6, 127.3, 127.9, 128.9, 129.9, 130.5, 135.4, 141.6, 172.4, 176.6; IR (ATR) 3185, 3063, 2940, 1718, 1656, 1610, 1464, 1343, 1167, 758, $714 \mathrm{~cm}^{-1}$; HRMS (ESI, positive) m/z for $\mathrm{C}_{19} \mathrm{H}_{18} \mathrm{~N}_{2} \mathrm{NaO}_{2}[\mathrm{M}+\mathrm{Na}]^{+}$: calcd. 329.1260, found 329.1264; HPLC (DAICEL CHIRALPAK IB-3®, hexane: $i-\mathrm{PrOH}=80: 20,1.0 \mathrm{~mL} / \mathrm{min}, 215 \mathrm{~nm}$ ), $\mathrm{tR}=17.0 \mathrm{~min}$ (major), $26.3 \mathrm{~min}$ (minor). 


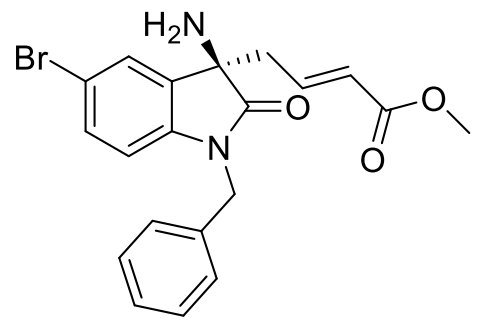

To a solution of $3 \mathbf{e}(27.5 \mathrm{mg}, 0.05 \mathrm{mmol})$ in dry dichloromethane $(1.3 \mathrm{~mL})$, anisole $(81.7 \mu \mathrm{L}, 0.75 \mathrm{mmol})$ at $0{ }^{\circ} \mathrm{C}$, then trifluoromethanesulfonic acid $(44.0 \mu \mathrm{L}, 0.50 \mathrm{mmol})$ was added slowly. The reaction mixture was stirred for $4 \mathrm{~h}$, then quenched with sat. $\mathrm{NaHCO}_{3}$, and aqueous layer was extracted with dichloromethane. The combined organic layer was dried over $\mathrm{Na}_{2} \mathrm{SO}_{4}$ and concentrated under reduced pressure to give the crude product, which was purified by silica gel column chromatography (hexane/ $\mathrm{CH}_{2} \mathrm{Cl}_{2} /$ ethyl acetate=2:1:1) to afford $\mathbf{8}(19.1 \mathrm{mg}, 92 \%$ yield) as a colorless oil. Ee was determined after transformation to 9.

$[\alpha]_{\mathrm{D}}{ }^{25}-43.8\left(\mathrm{c} 0.33, \mathrm{CHCl}_{3}\right) ;{ }^{1} \mathrm{H}$ NMR $\left(300 \mathrm{MHz}, \mathrm{CDCl}_{3}\right) \delta 1.79(\mathrm{br}, 2 \mathrm{H}), 2.67-2.82(\mathrm{~m}, 2 \mathrm{H}), 3.69$ (s, $3 \mathrm{H}), 4.67(\mathrm{~d}, J=15.8 \mathrm{~Hz}, 1 \mathrm{H}), 5.04(\mathrm{~d}, J=15.8 \mathrm{~Hz}, 1 \mathrm{H}), 5.87(\mathrm{~d}, J=15.6 \mathrm{~Hz}, 1 \mathrm{H}), 6.57(\mathrm{~d}, J=8.4 \mathrm{~Hz}$, $1 \mathrm{H}), 6.61-6.71(\mathrm{~m}, 1 \mathrm{H}), 7.21(\mathrm{~d}, J=7.8 \mathrm{~Hz}, 2 \mathrm{H}), 7.26-7.34(\mathrm{~m}, 4 \mathrm{H}), 7.50(\mathrm{~d}, J=2.1 \mathrm{~Hz}, 1 \mathrm{H}) ;{ }^{13} \mathrm{C} \mathrm{NMR}$ $\left(125 \mathrm{MHz} \mathrm{CDCl}_{3}\right) \delta 41.6,43.9,51.6,60.7,111.1,115.9,125.7,127.1,127.2,127.8,128.9,132.3,132.5$, 134.9, 140.9, 141.2, 166.0, 179.0; IR (ATR) 3362, 3032, 2950, 1712, 1605, 1482, 1331, 1173, 809, 729, $697 \mathrm{~cm}^{-1}$; HRMS (ESI, positive) m/z for $\mathrm{C}_{20} \mathrm{H}_{19} \mathrm{BrN}_{2} \mathrm{NaO}_{3}[\mathrm{M}+\mathrm{Na}]^{+}$: calcd. 437.0471, found 437.0471 .

\section{Methyl (R,E)-4-[1-benzyl-5-bromo-3-(4-methoxybenzenesulfonamide)-2-oxoindolin-3-yl]but-2- enoate (9)}

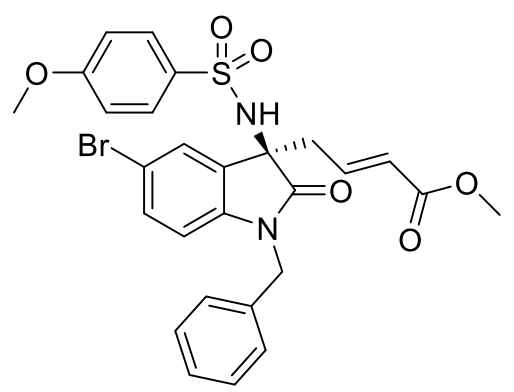

To a solution of $8(27.5 \mathrm{mg}, 8.7 \mu \mathrm{mol})$ in dry chloroform $(44.0 \mu \mathrm{L})$, pyridine $(1.05 \mu \mathrm{L}, 13.0 \mu \mathrm{mol})$ at $0{ }^{\circ} \mathrm{C}$, then 4-methoxybenzenesulfonyl

Chloride $(2.2 \mathrm{mg}, 10.0 \mu \mathrm{mol})$ was added. The reaction mixture was warmed up to r.t. and stirred for $26 \mathrm{~h}$, then quenched with $\mathrm{H}_{2} \mathrm{O}$, and aqueous layer was extracted with dichloromethane. The combined organic layer was dried over $\mathrm{Na}_{2} \mathrm{SO}_{4}$ and concentrated under reduced pressure to give the crude product, which was purified by silica gel column chromatography (hexane $/ \mathrm{CH}_{2} \mathrm{Cl}_{2} /$ ethyl acetate $\left.=2: 1: 1\right)$ to afford $9(5.1 \mathrm{mg}, 99 \%$ yield, $95 \%$ ee) as a white solid. It was used $\mathrm{X}$-ray crystal analysis to determine absolute stereochemistry.

$[\alpha]_{\mathrm{D}}{ }^{25}-121.8\left(\mathrm{c} 0.12, \mathrm{CHCl}_{3}\right)$; m.p. 177.0-178.0 ${ }^{\circ} \mathrm{C} ;{ }^{1} \mathrm{H} \mathrm{NMR}\left(500 \mathrm{MHz}, \mathrm{CDCl}_{3}\right) \delta 2.75-2.85(\mathrm{~m}, 2 \mathrm{H})$, $3.68(\mathrm{~s}, 3 \mathrm{H}), 3.87(\mathrm{~s}, 3 \mathrm{H}), 4.73(\mathrm{~d}, J=16.0 \mathrm{~Hz}, 1 \mathrm{H}), 5.02(\mathrm{~d}, J=16.0 \mathrm{~Hz}, 1 \mathrm{H}), 5.92(\mathrm{~d}, J=15.5 \mathrm{~Hz}, 1 \mathrm{H})$, 6.05 (s, 1H), 6.44-6.49 (m, 1H), 6.51 (d, J=8.5 Hz, 1H), 6.65 (d, J=2.0 Hz, 1H), 6.79-6.82 (m, 2H), 7.23 $(\mathrm{dd}, J=2.0,8.5 \mathrm{~Hz}, 1 \mathrm{H}), 7.25-7.36(\mathrm{~m}, 7 \mathrm{H}) ;{ }^{13} \mathrm{C} \mathrm{NMR}\left(125 \mathrm{MHz}, \mathrm{CDCl}_{3}\right) \delta$ 40.8, 44.6, 51.7, 55.6, 62.4, $111.3,113.9,115.3,126.4,127.1,127.2$, 127.8, 128.1, 128.9, 129.5, 132.0, 132.6, 134.8, 138.4, 142.1, 163.1, 165.7, 175.7; IR (ATR) 3230, 2948, 2841, 1717, 1596, 1330, 1260, 1151, 1091, 728, $697 \mathrm{~cm}^{-1}$; HRMS (ESI, positive) $\mathrm{m} / \mathrm{z}$ for $\mathrm{C}_{27} \mathrm{H}_{25} \mathrm{BrN}_{2} \mathrm{NaO}_{6} \mathrm{~S}[\mathrm{M}+\mathrm{Na}]^{+}$: calcd. 607.0509, found 607.0507; HPLC 
(DAICEL CHIRALPAK IA ${ }^{\circledR}$, hexane:ethyl acetate $=70: 30,1.0 \mathrm{~mL} / \mathrm{min}, 254 \mathrm{~nm}$ ), $\mathrm{tR}=23.4$ min (major), $43.4 \mathrm{~min}$ (minor). 
Figure S1. ESI-Mass spectroscopic analysis for complex between $4 a$ and $\mathrm{Cu}(\mathrm{OTf})_{2}$ with ketimine 1d

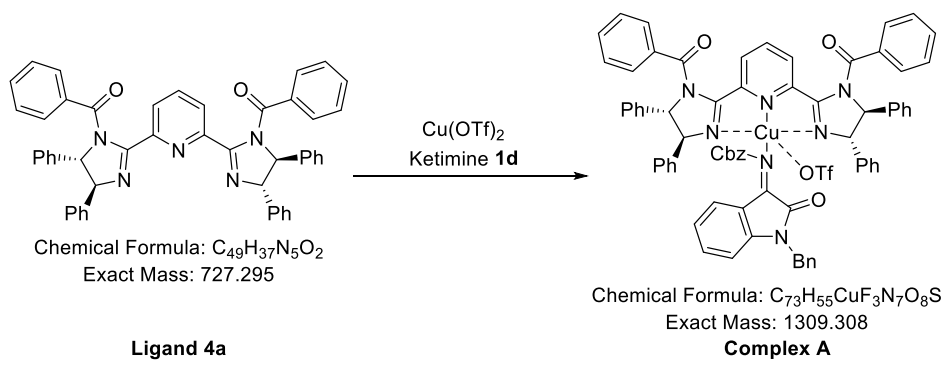

(a) The ESI-Mass spectrum of ligand $\mathbf{4 a}$, ketimine $\mathbf{1 d}$ and $\mathrm{Cu}(\mathrm{OTf})_{2}$.

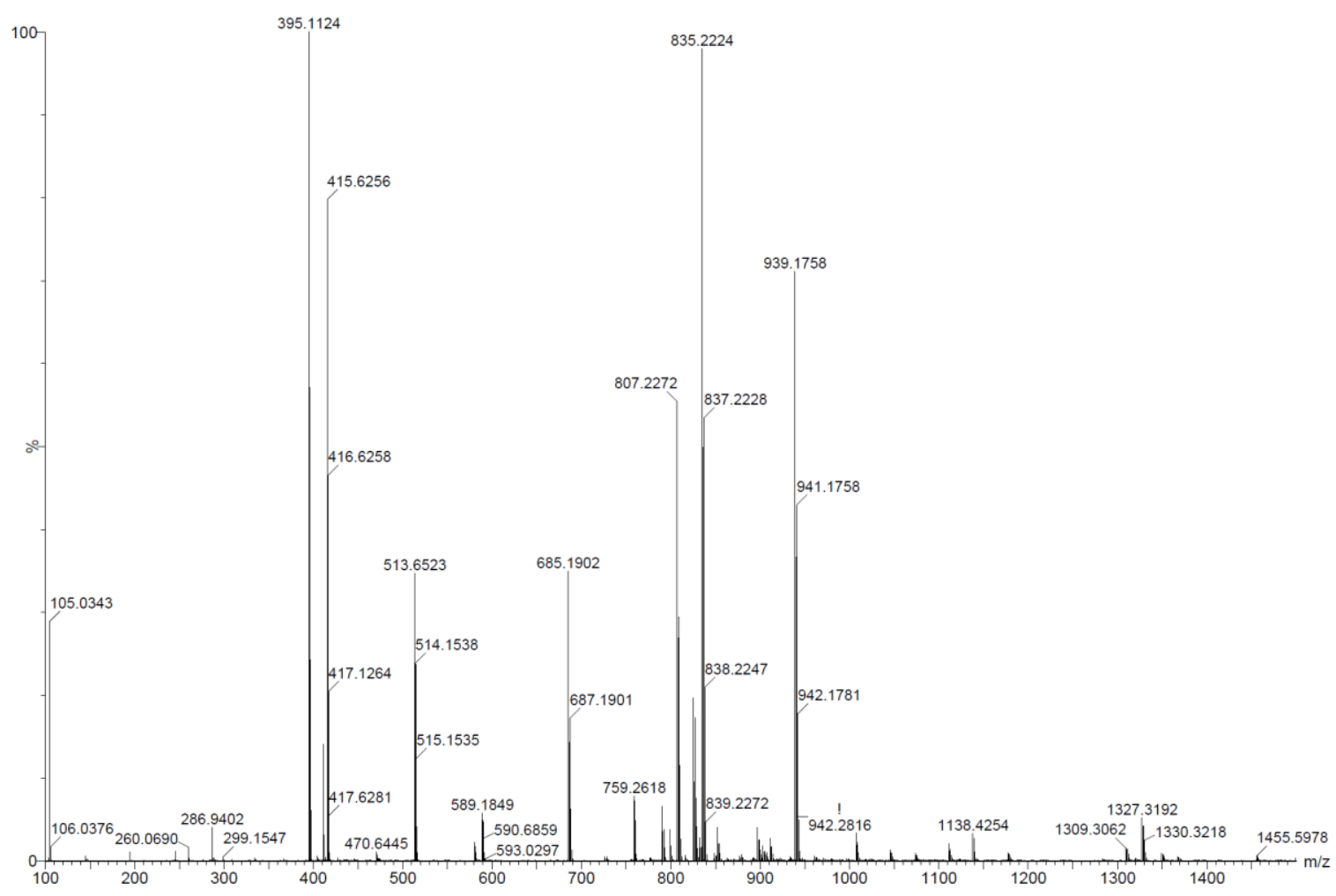


(b) Theoretical peaks about Complex $\mathbf{A}$

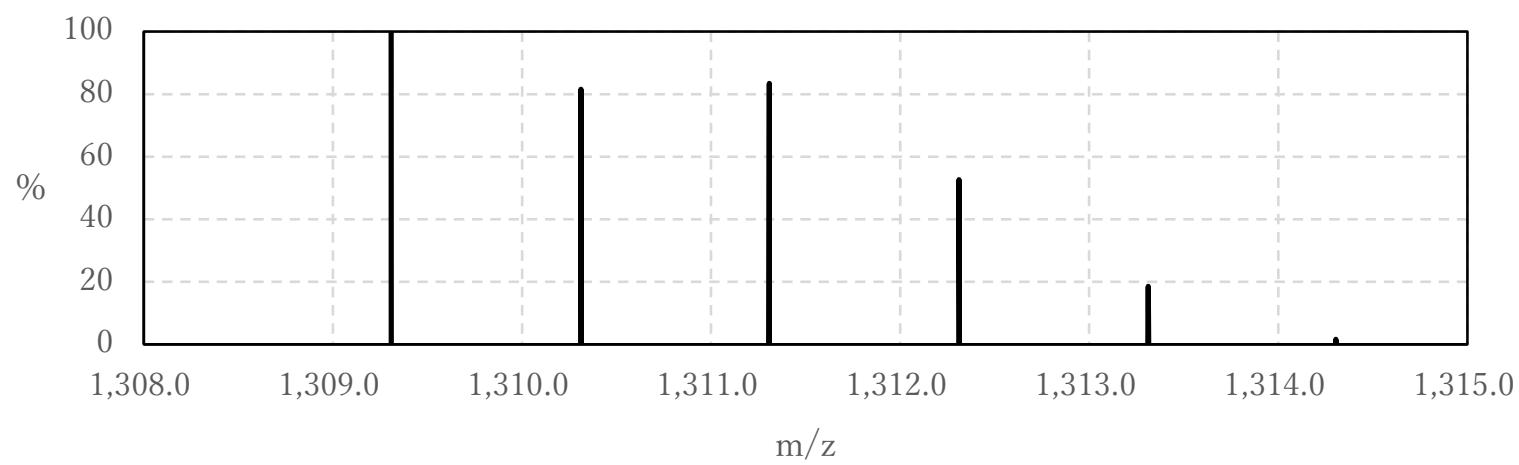

(c) Major peaks about Complex A

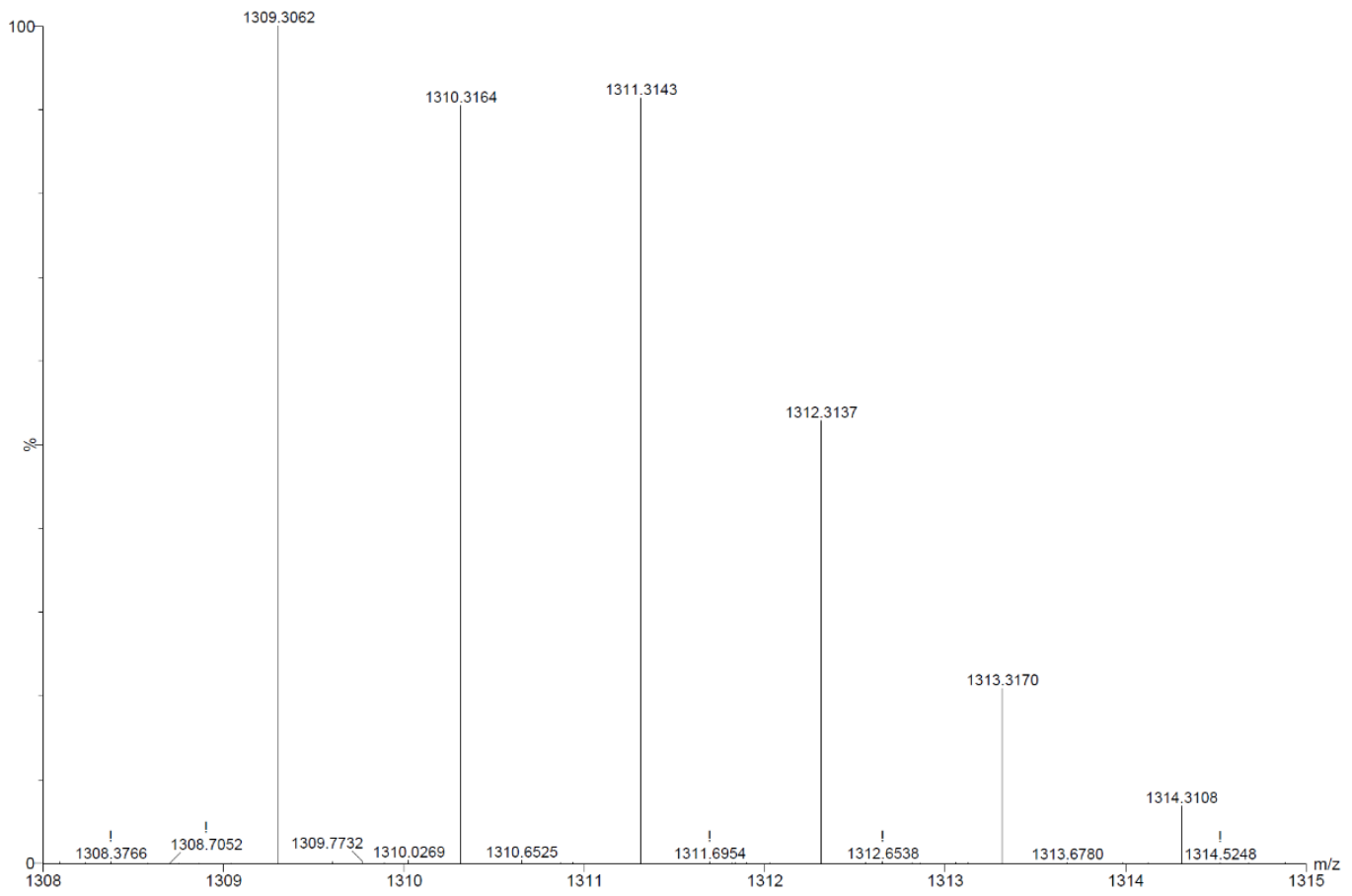




\section{References}

1) (a) Anilkumar, G.; Bhor, S.; Tse, M. K.; Kkawonn, M.; Bitterlich, B.; Beller, M. Tetrahedron Asymmetry, 2005, 16, 3536. (b) Nakamura, S.; Ohara, M.; Nakamura, Y.; Shibata, N.; Toru, T. Chem. Eur. J. 2010, 16, 2360.

2) Ratjen, L.; Garcia-Garcia, P.; Lay, F.; Beck, M. E.; List, B. Angew. Chem. Int. Ed. 2011, 50, 754.

3) Yan, W.; Wang, D.; Feng, J.; Li, P.; Zhao, D.; Wang, R. Org. Lett. 2012, 14, 2512. 


\section{${ }^{1} \mathrm{H}$ and ${ }^{13} \mathrm{C}$ NMR}

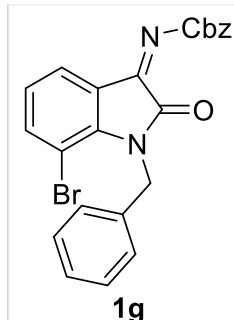

${ }^{1} \mathrm{H}$ NMR

$\left(\mathrm{CDCl}_{3}, 300 \mathrm{MHz}\right)$
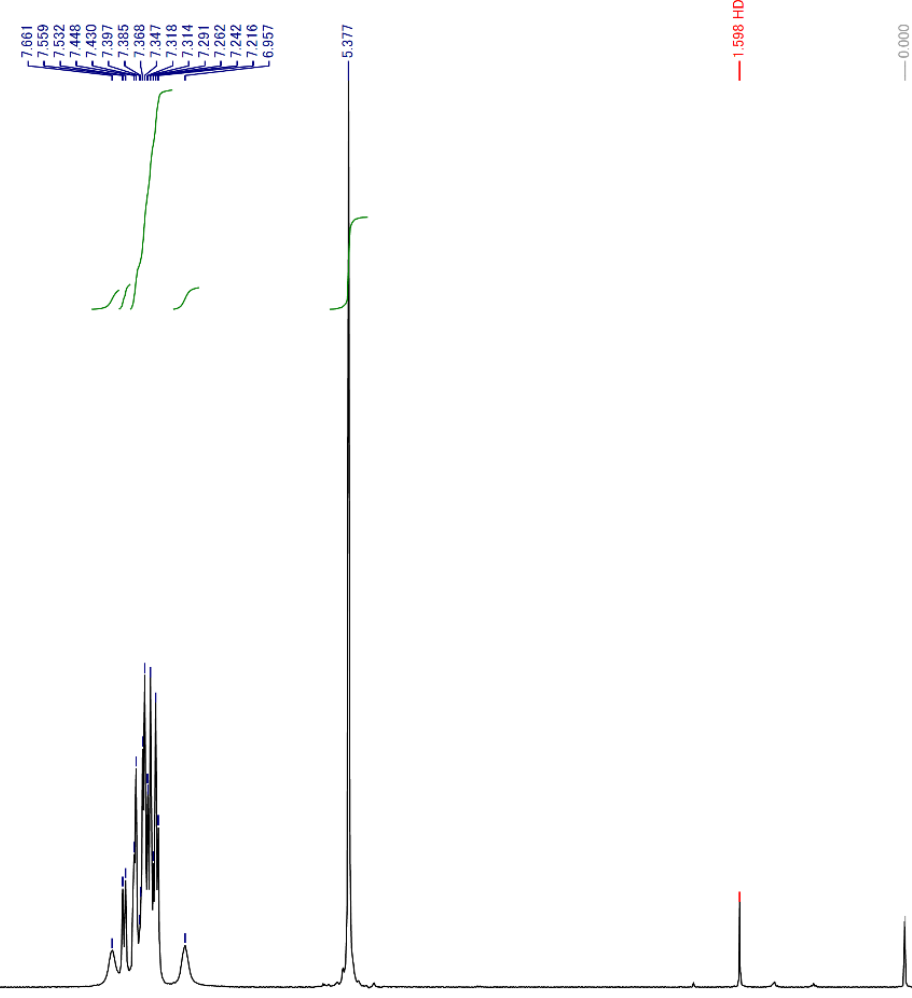

萼
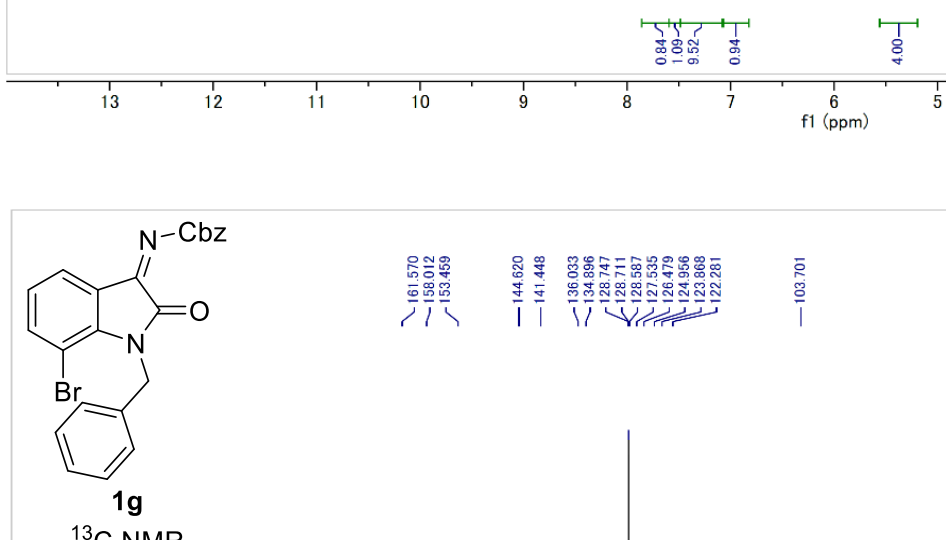

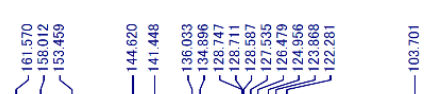

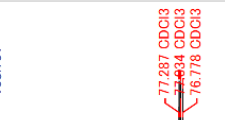

0
0

( $\mathrm{CDCl}_{3}, 125 \mathrm{MHz}$ )

$\left(\mathrm{CDCl}_{3}, 125 \mathrm{MHz}\right)$
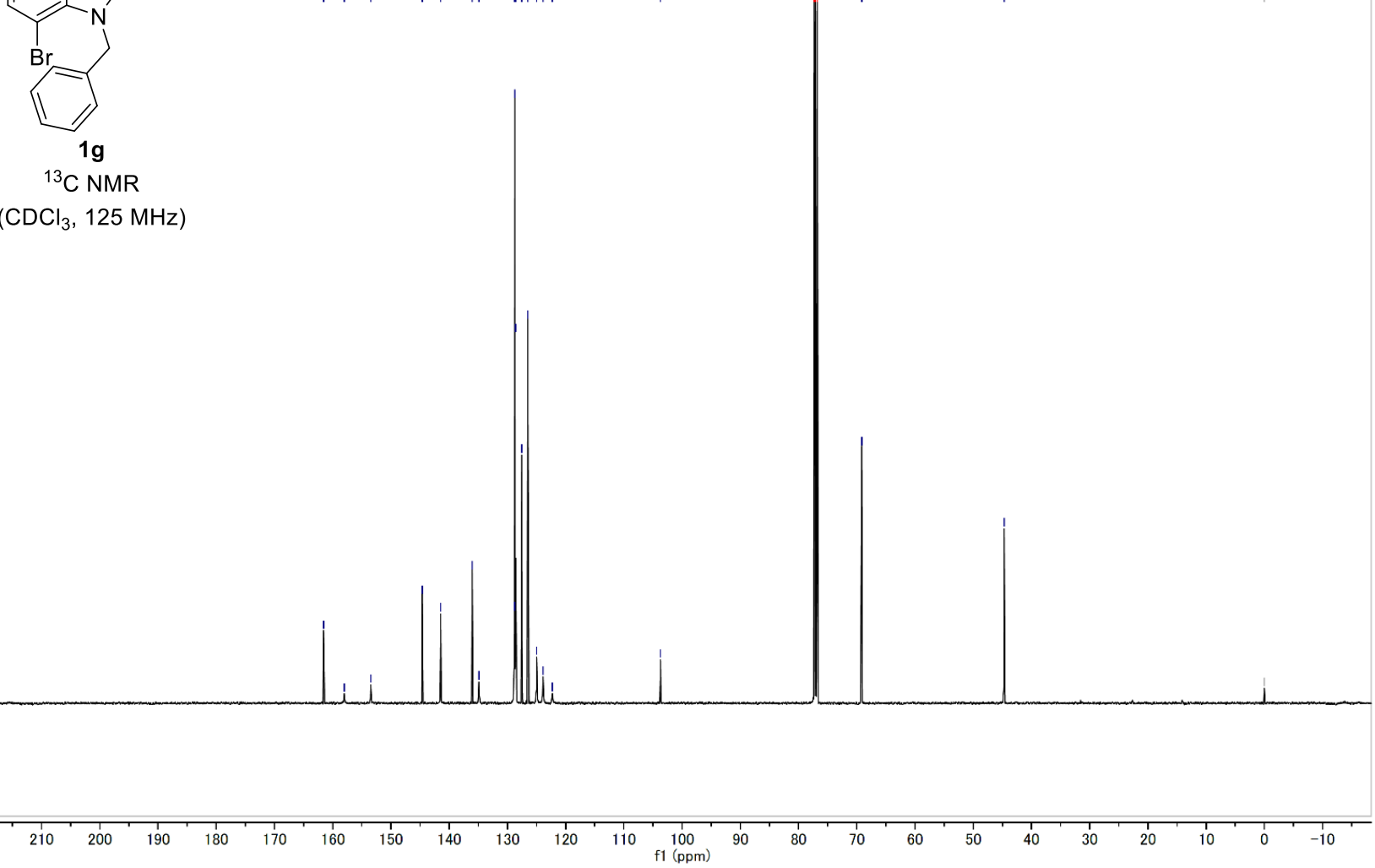

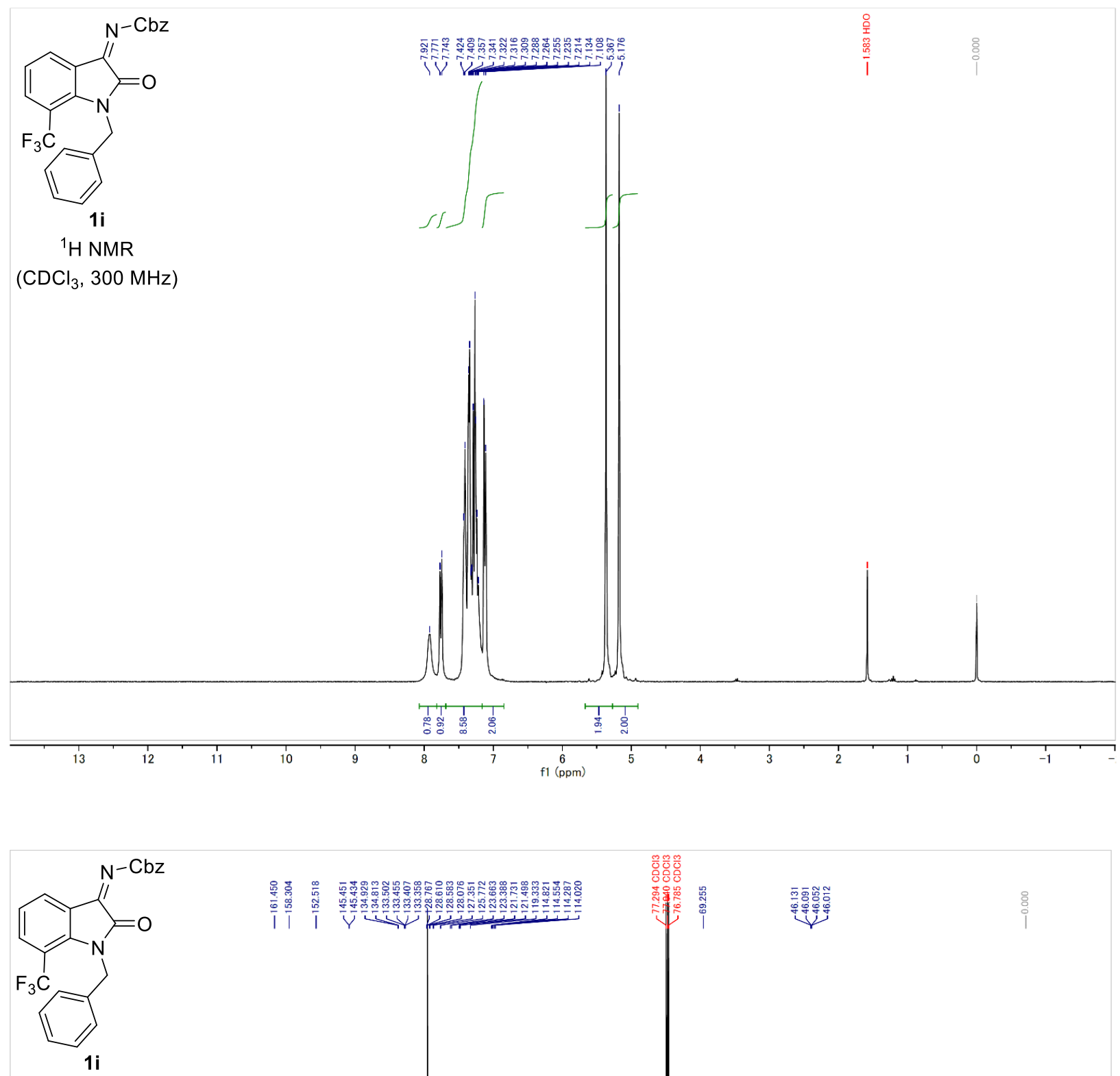

${ }^{13} \mathrm{C}$ NMR

$\left(\mathrm{CDCl}_{3}, 125 \mathrm{MHz}\right)$

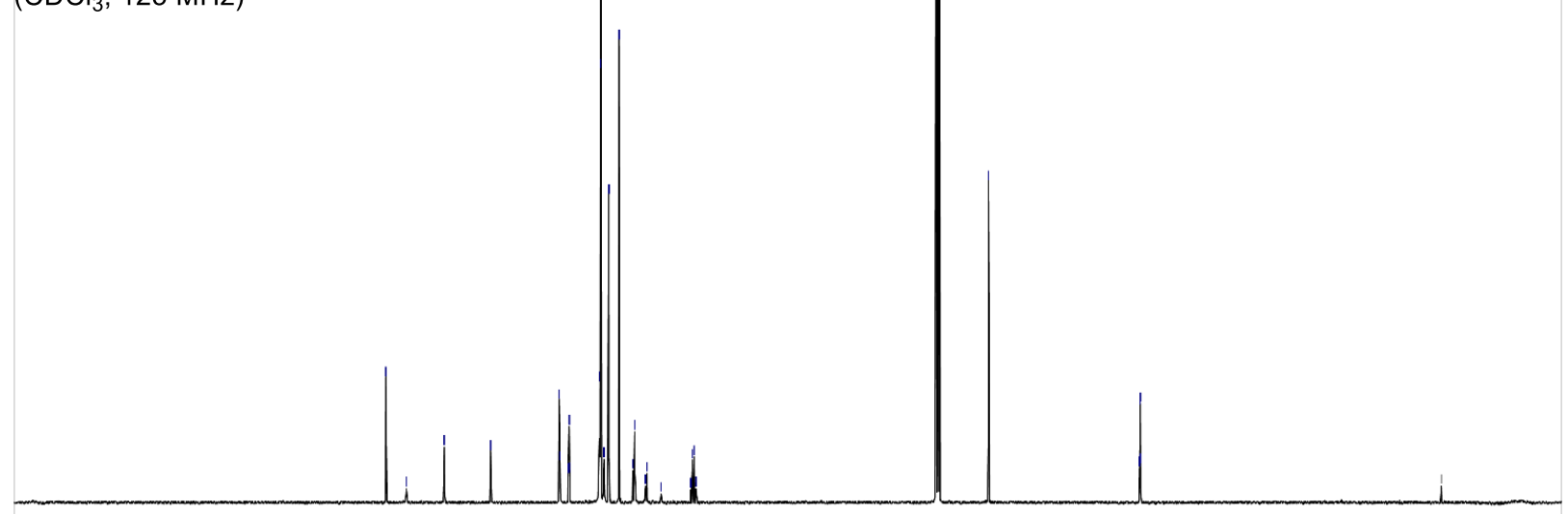

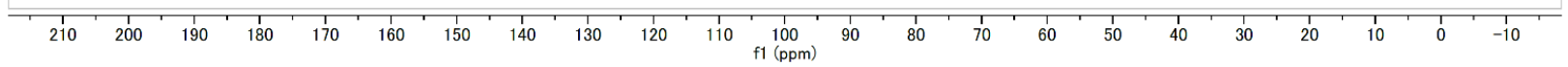




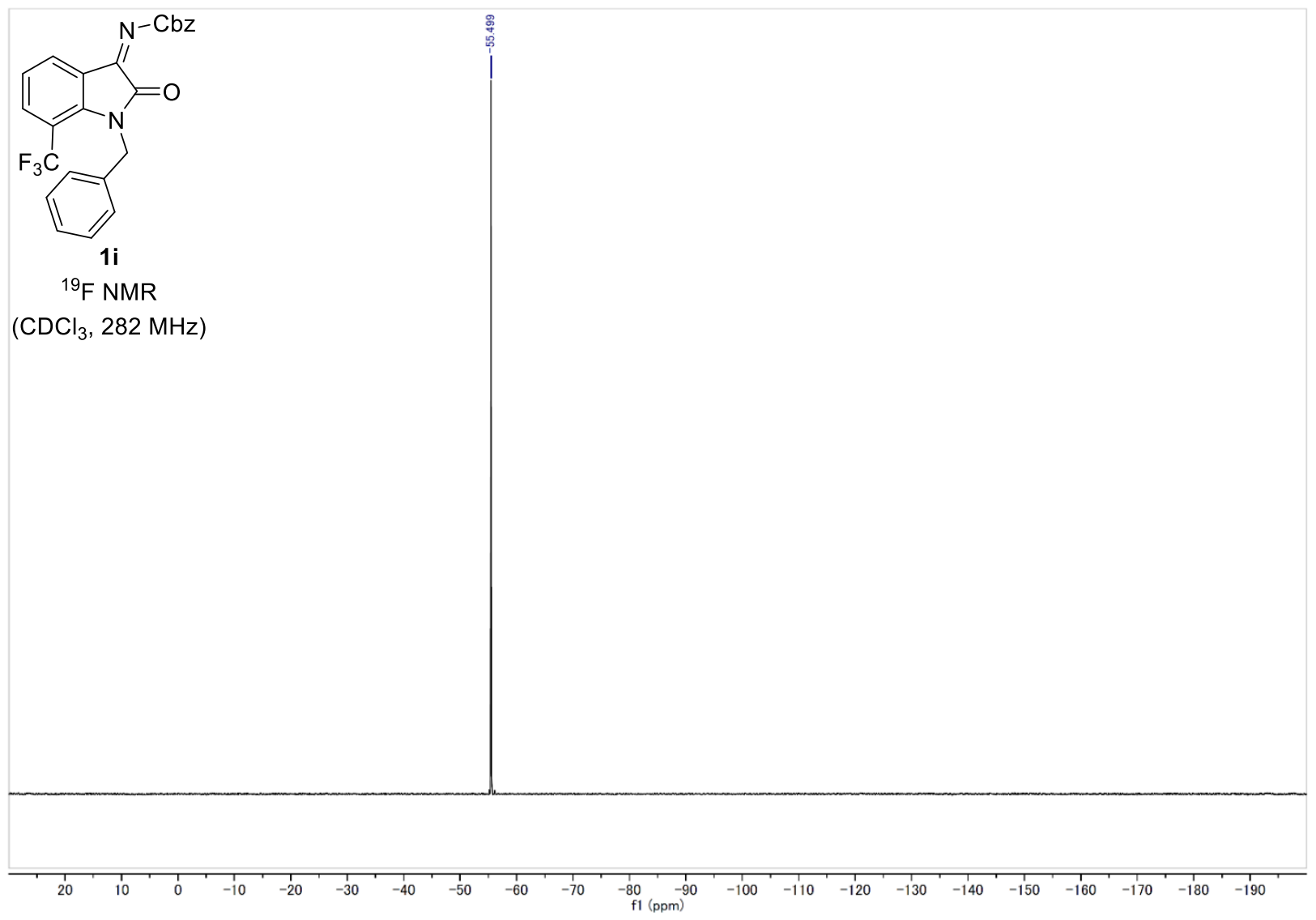




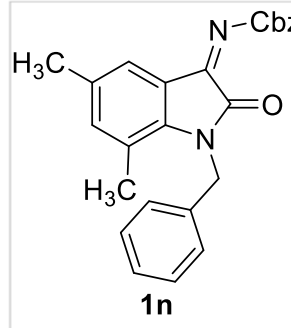

${ }^{1} \mathrm{H}$ NMR
$\left(\mathrm{CDCl}_{3}, 300 \mathrm{MHz}\right)$

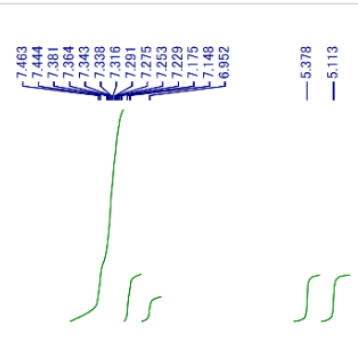

$\underset{0}{\infty}$

(

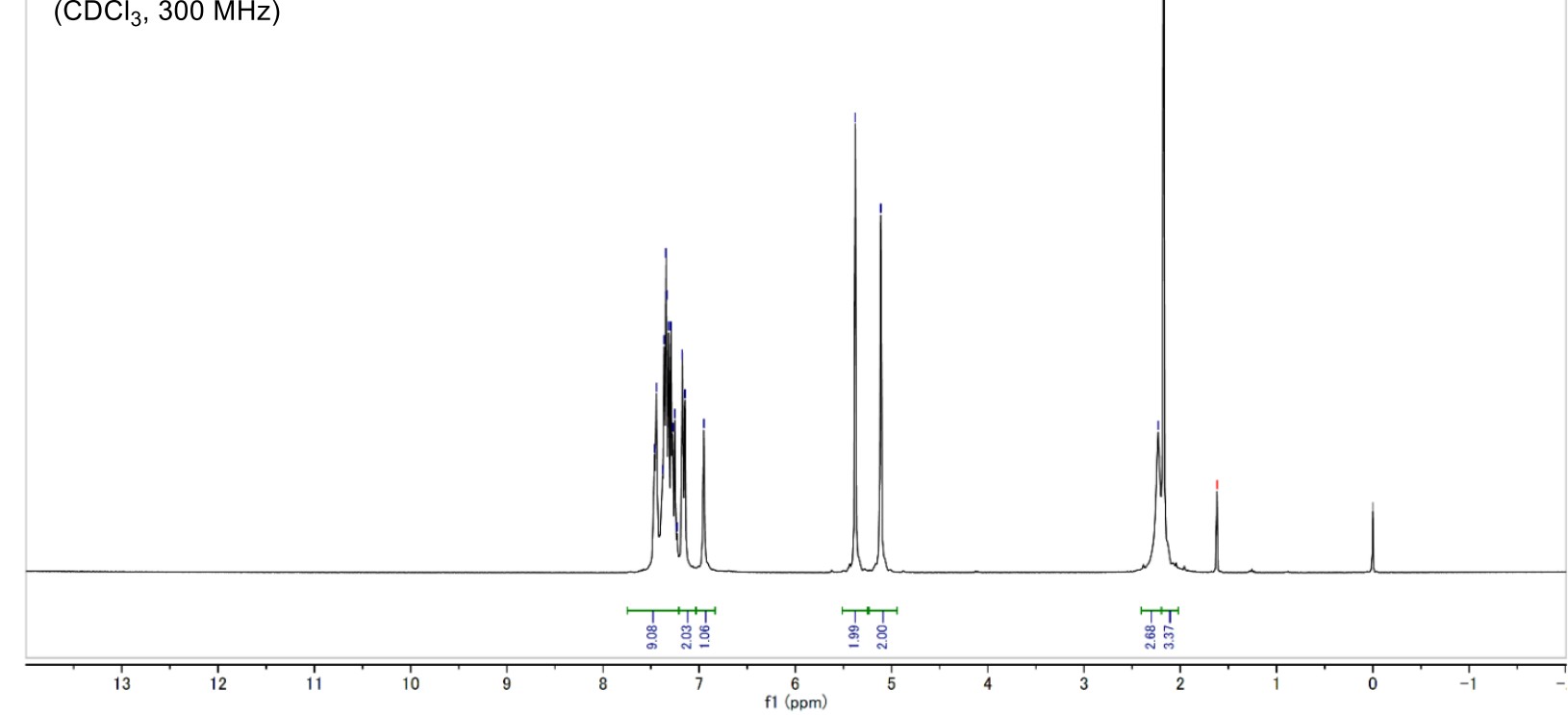

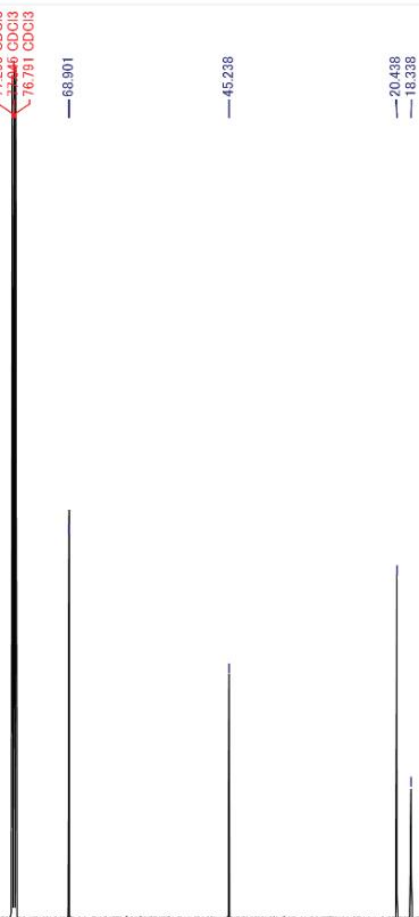

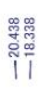

$\left(\mathrm{CDCl}_{3}, 125 \mathrm{MHz}\right)$

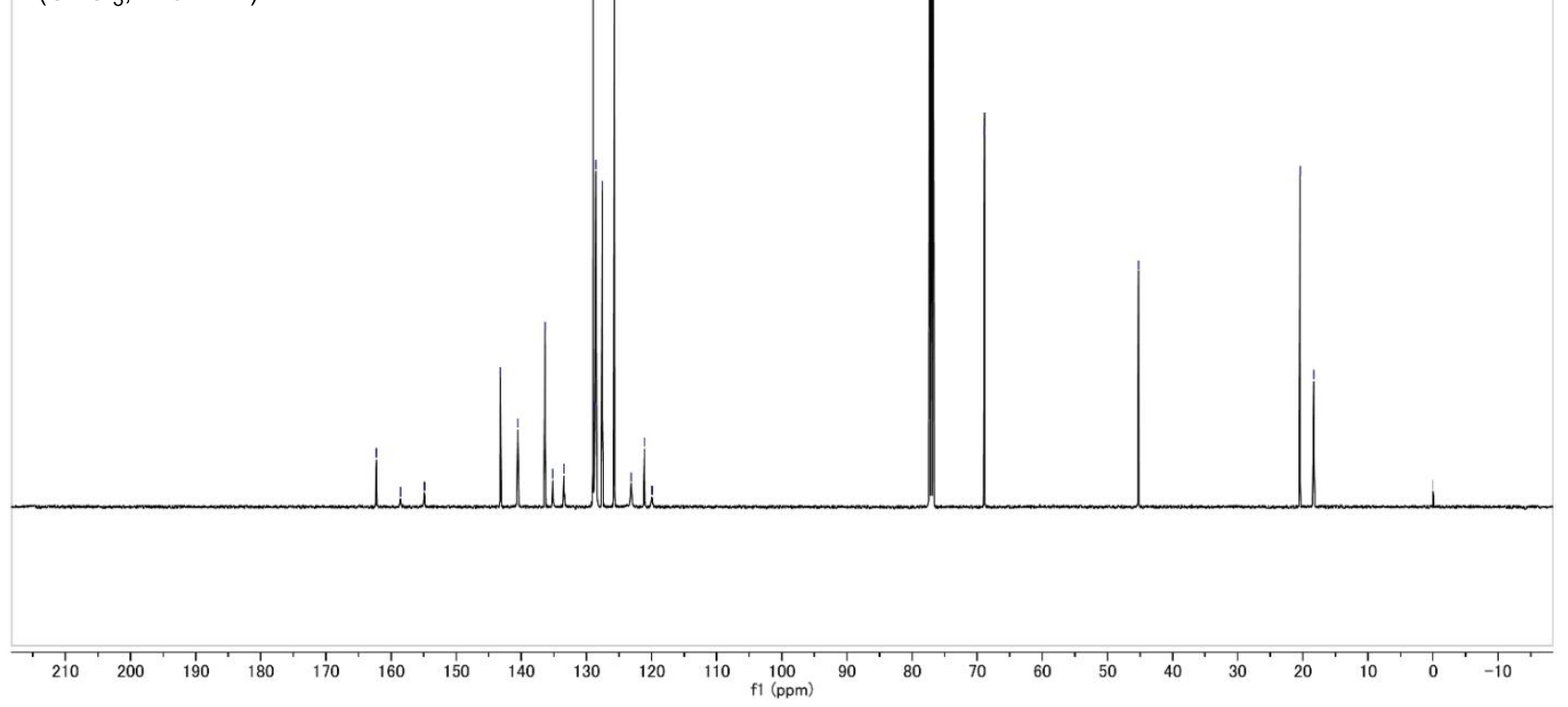




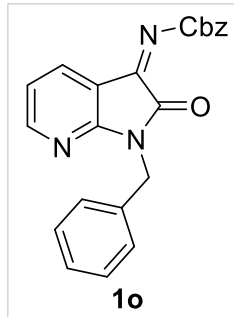

${ }^{1} \mathrm{H}$ NMR

$\left(\mathrm{CDCl}_{3}, 300 \mathrm{MHz}\right)$
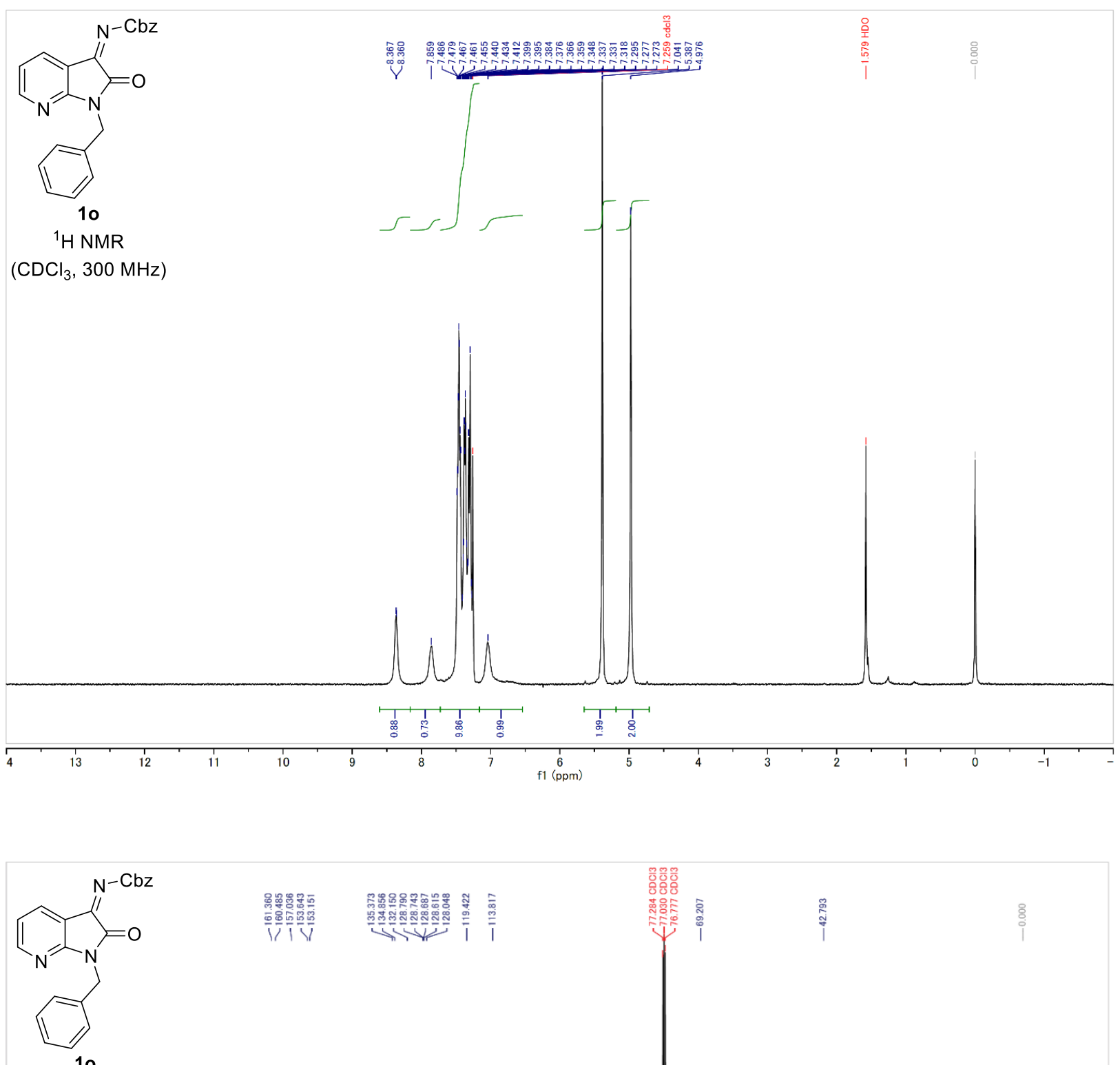

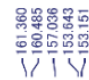

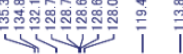

${ }^{13} \mathrm{C}$ NMR

$\left(\mathrm{CDCl}_{3}, 125 \mathrm{MHz}\right)$
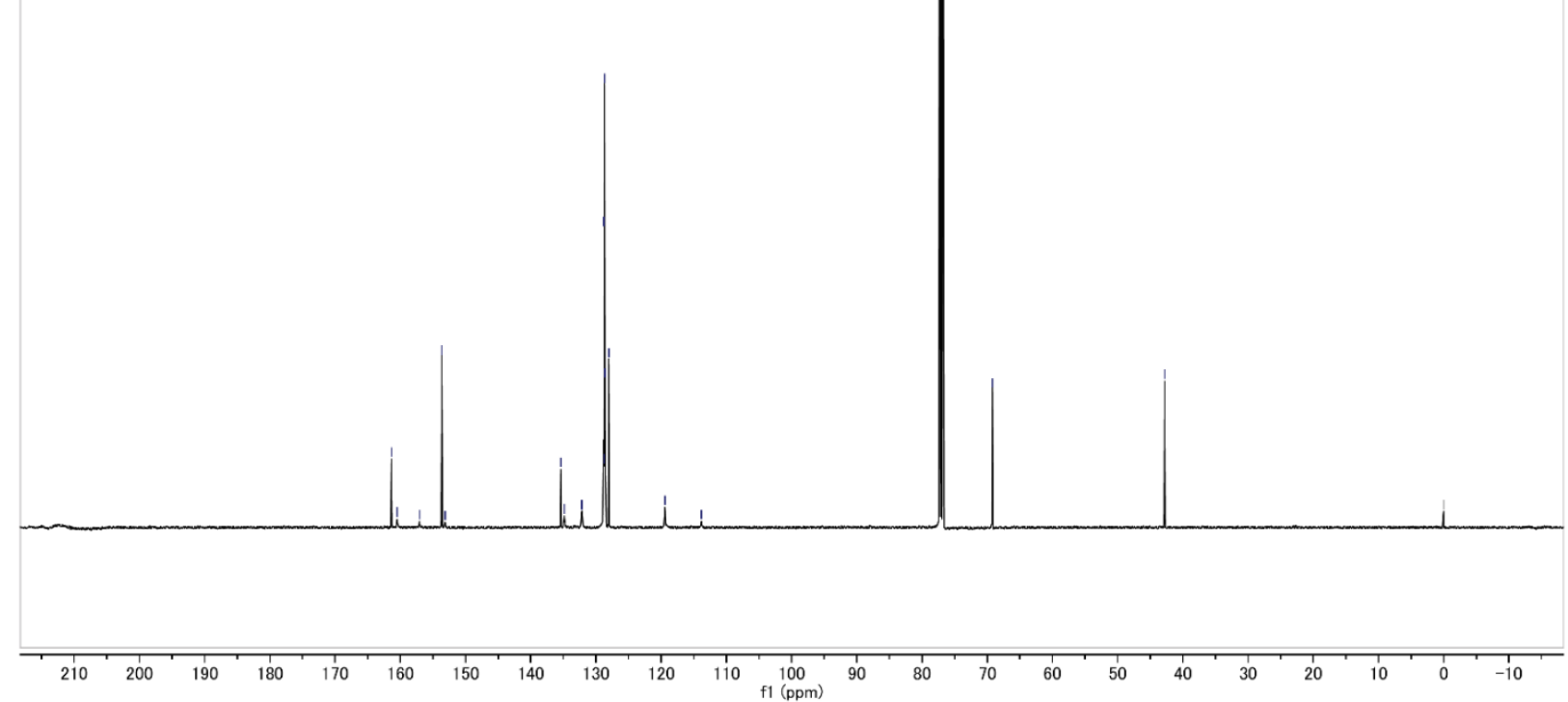

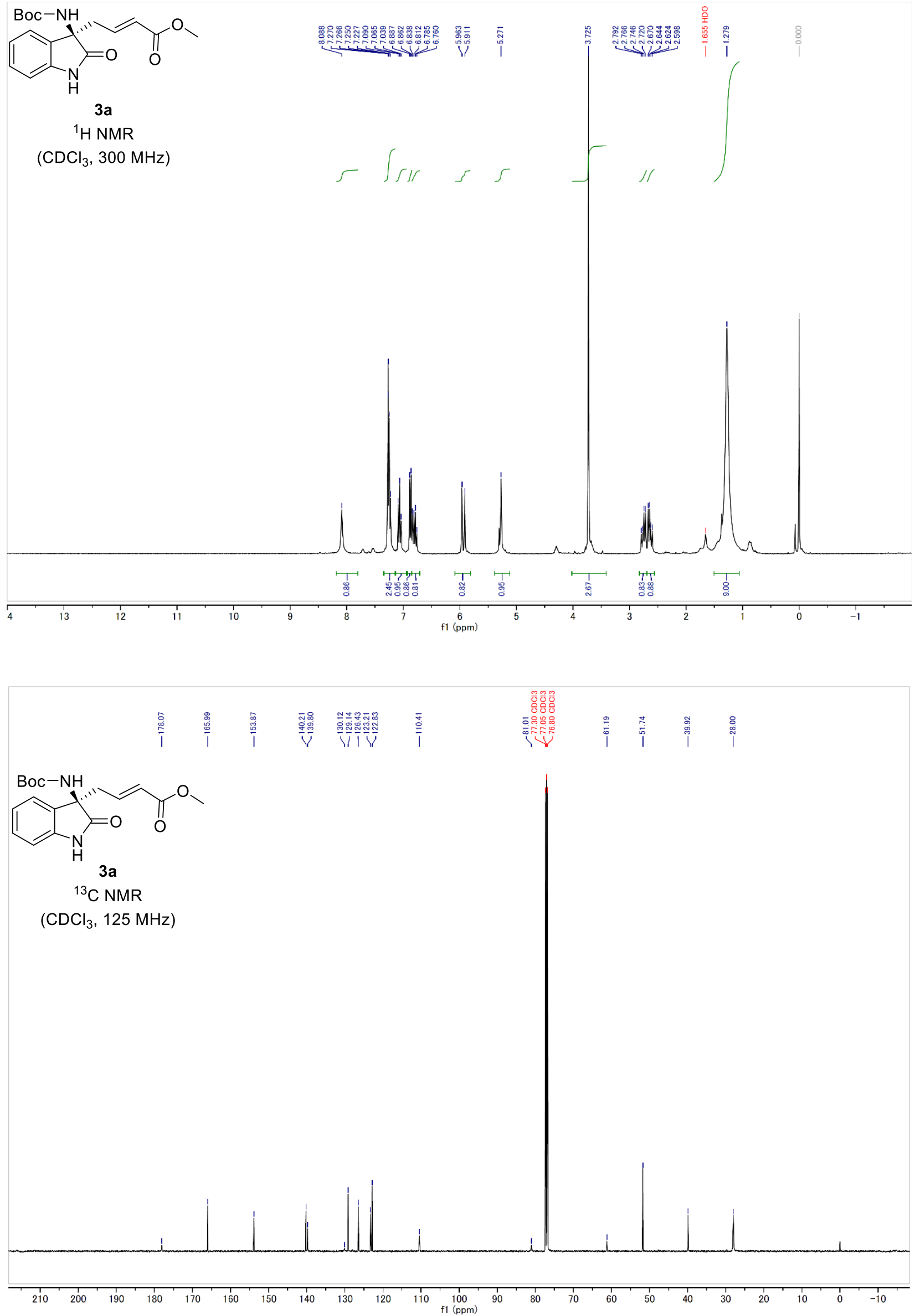

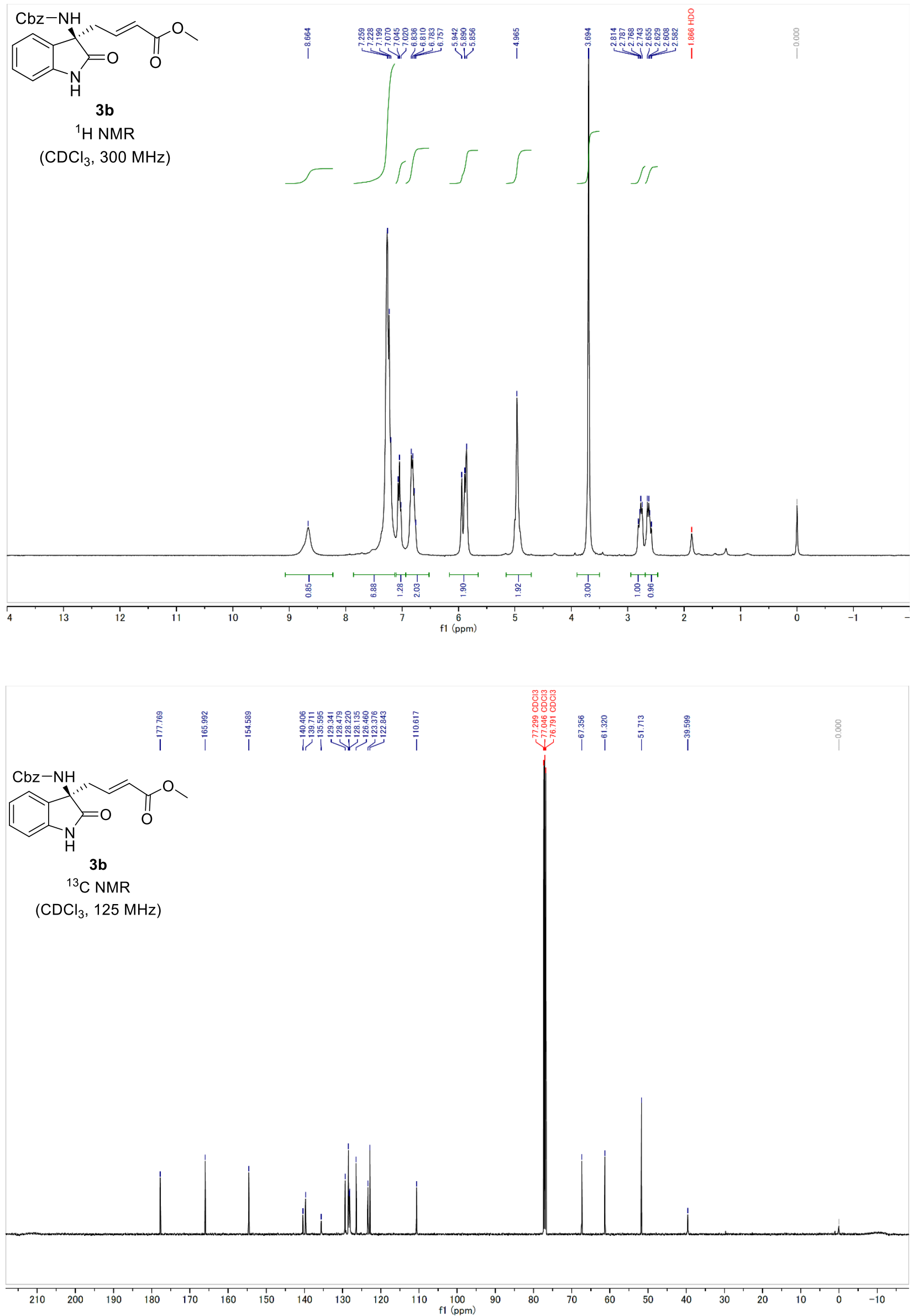


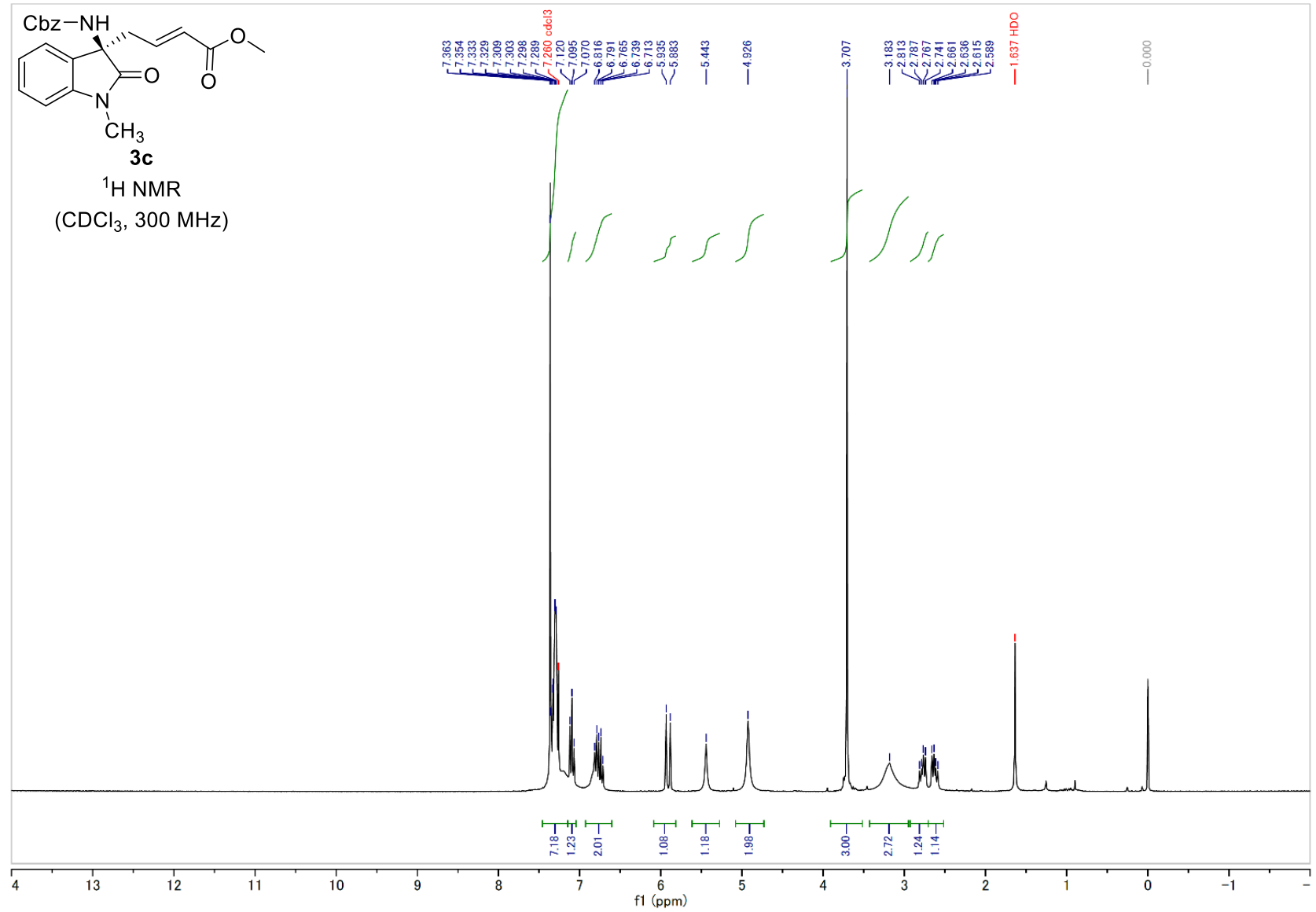

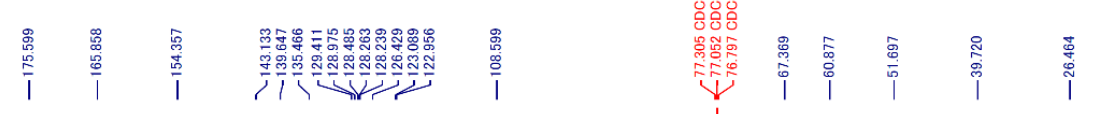<smiles>COC(=O)/C=C/C[C@@]1(NC(=O)O)C(=O)N(C)c2ccccc21</smiles>

${ }^{13} \mathrm{C}$ NMR

$\left(\mathrm{CDCl}_{3}, 125 \mathrm{MHz}\right)$

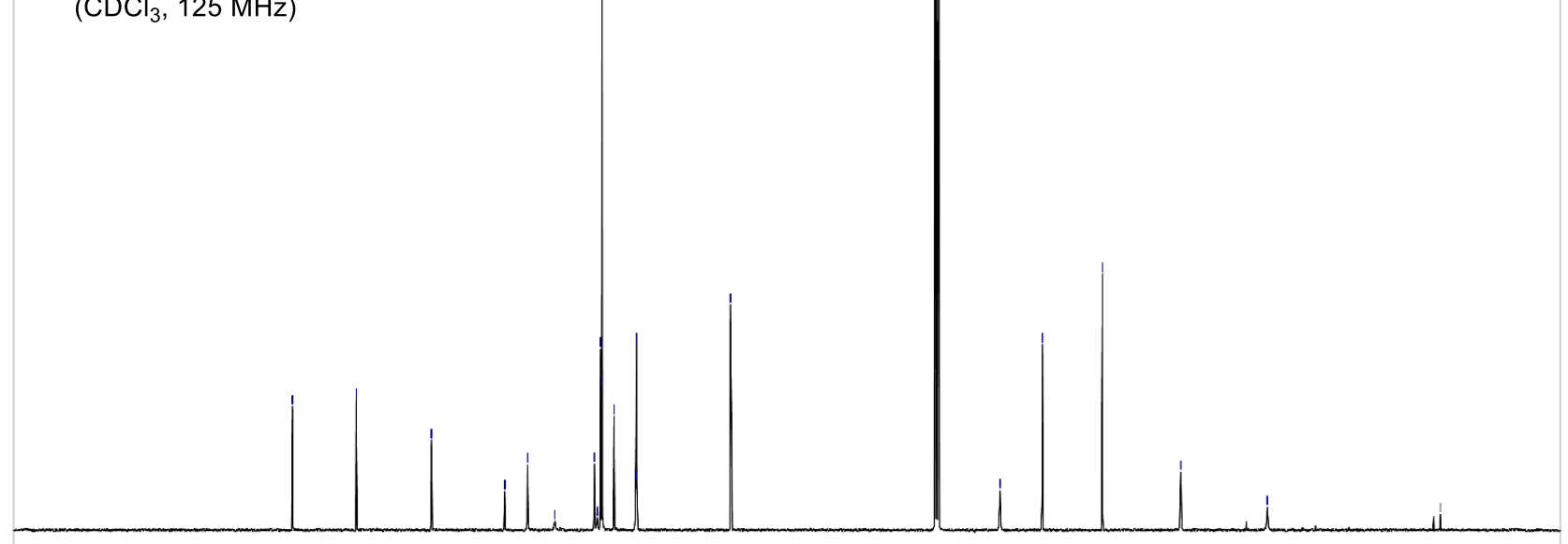

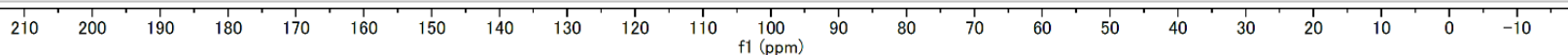



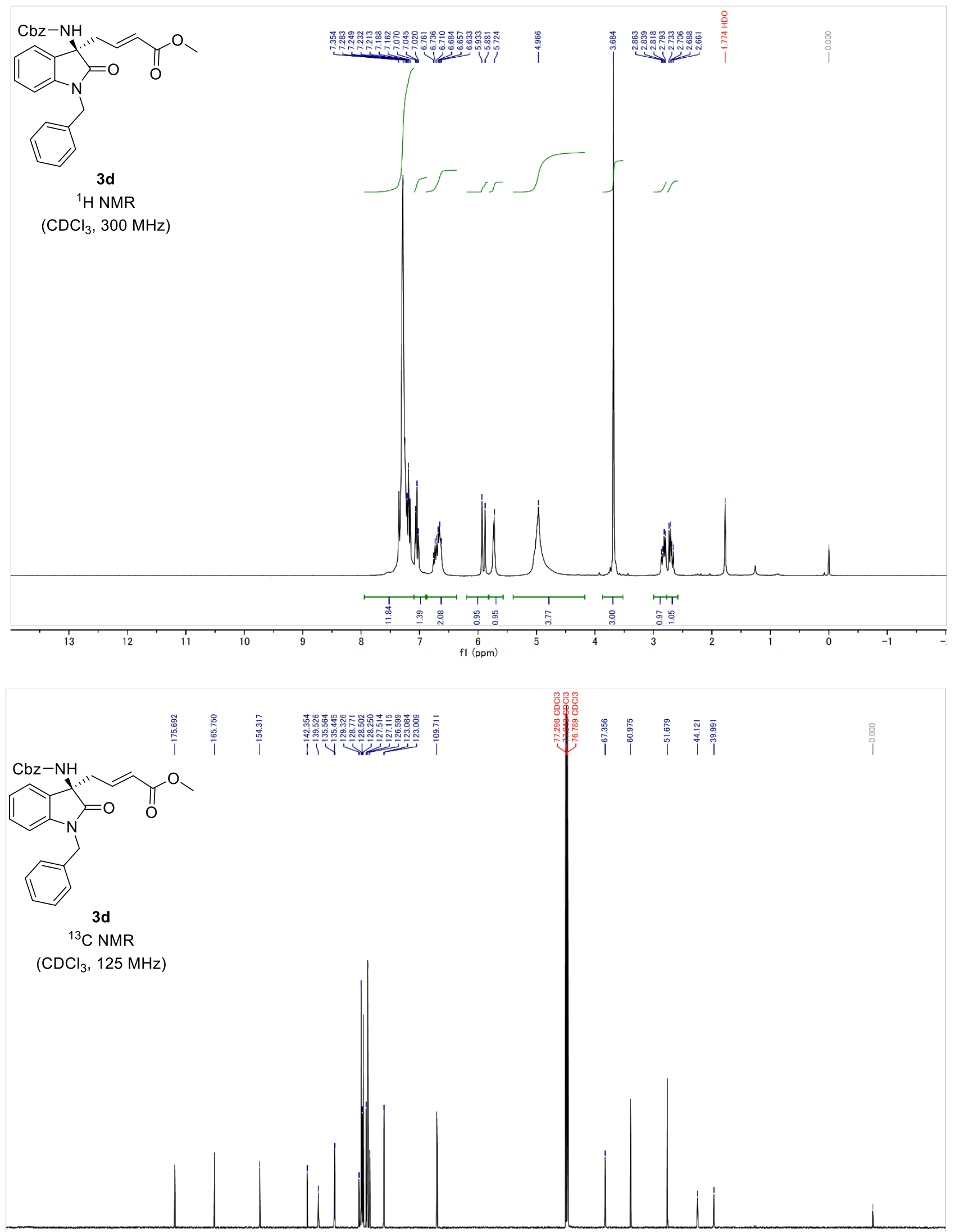

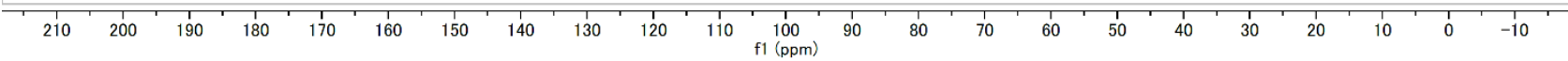



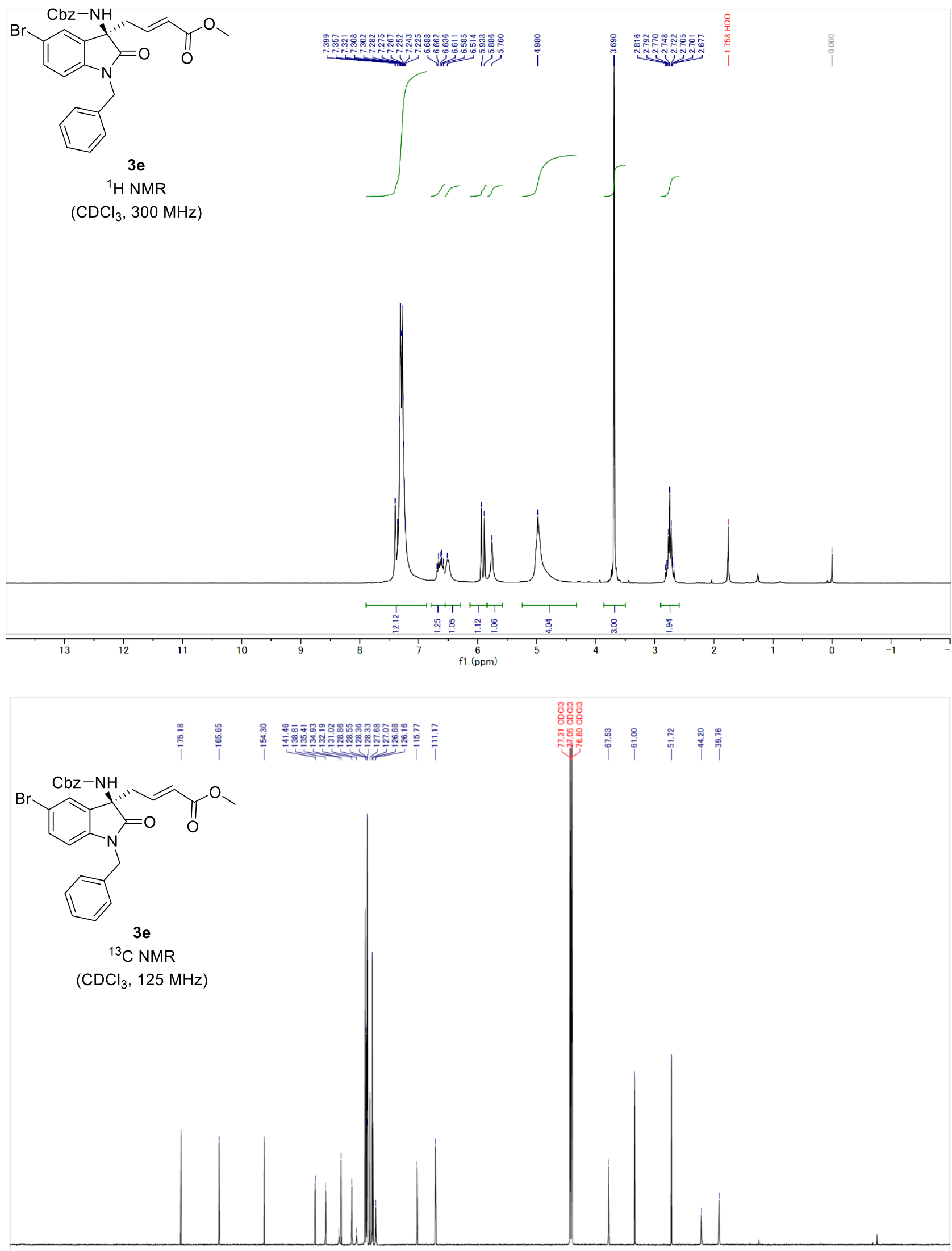

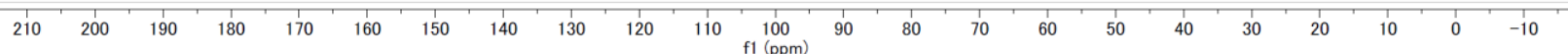



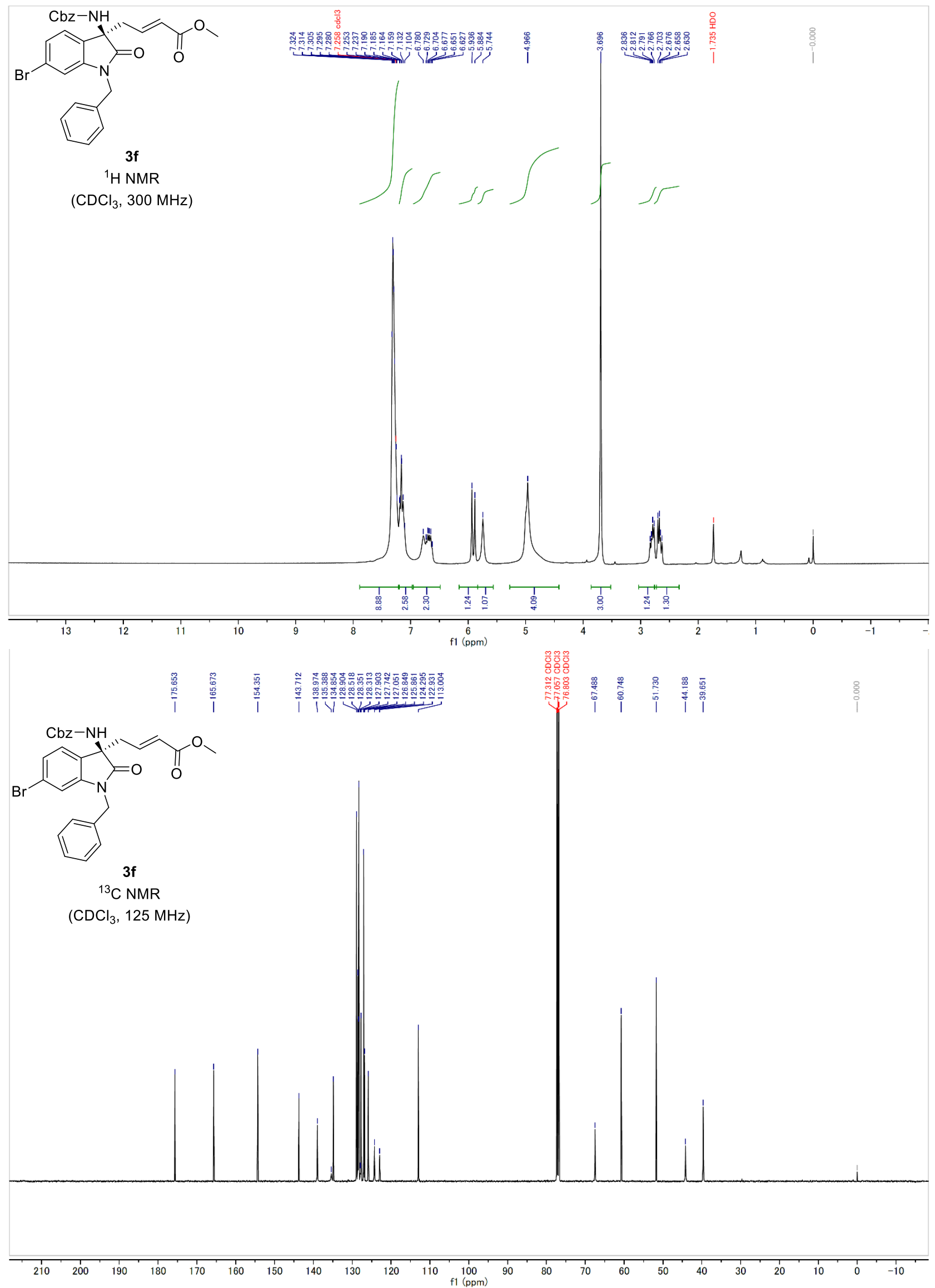

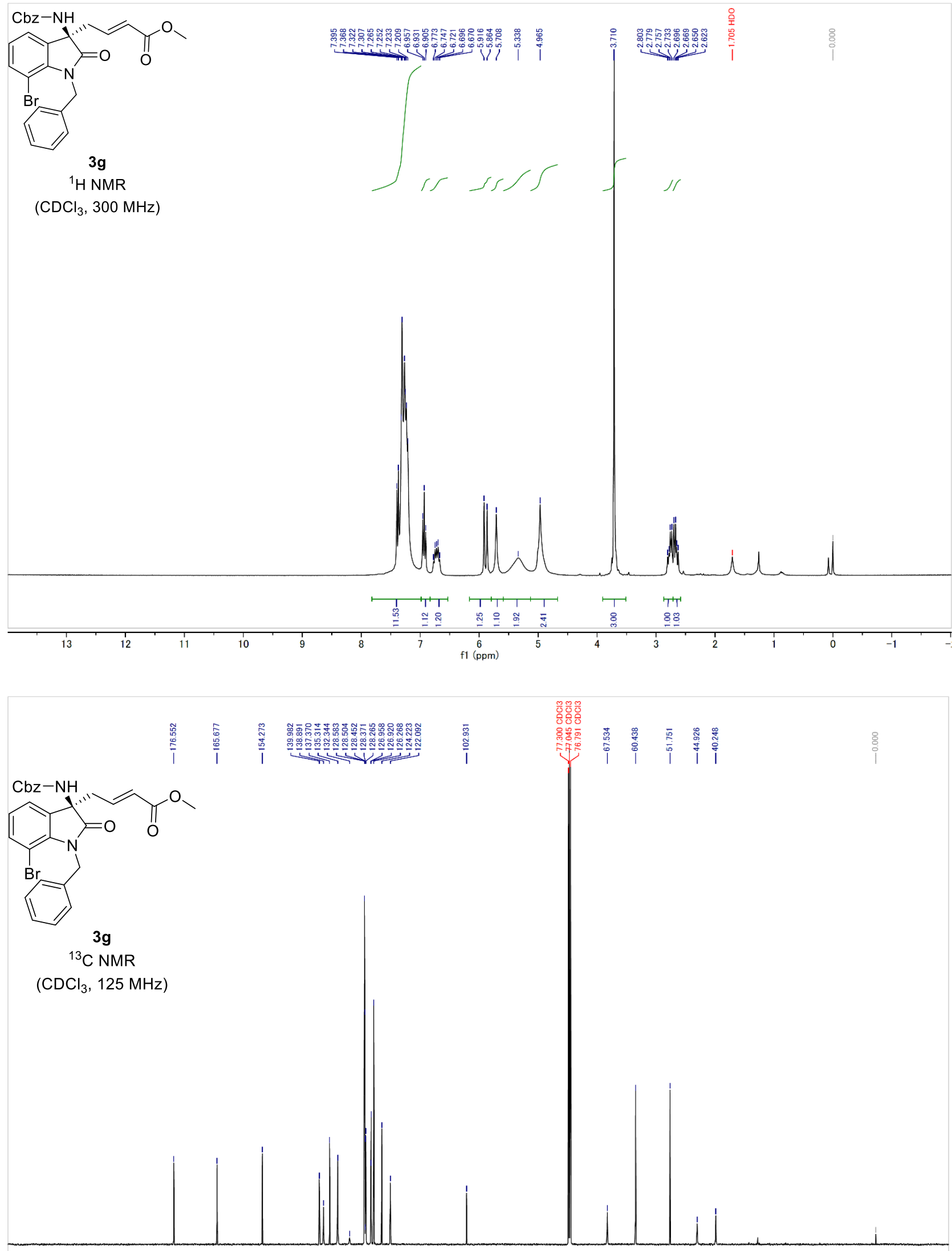

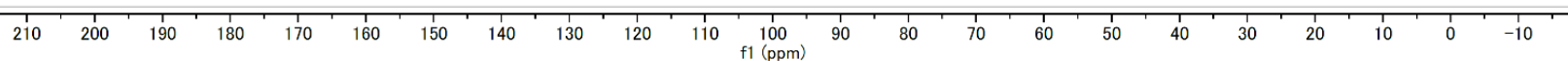



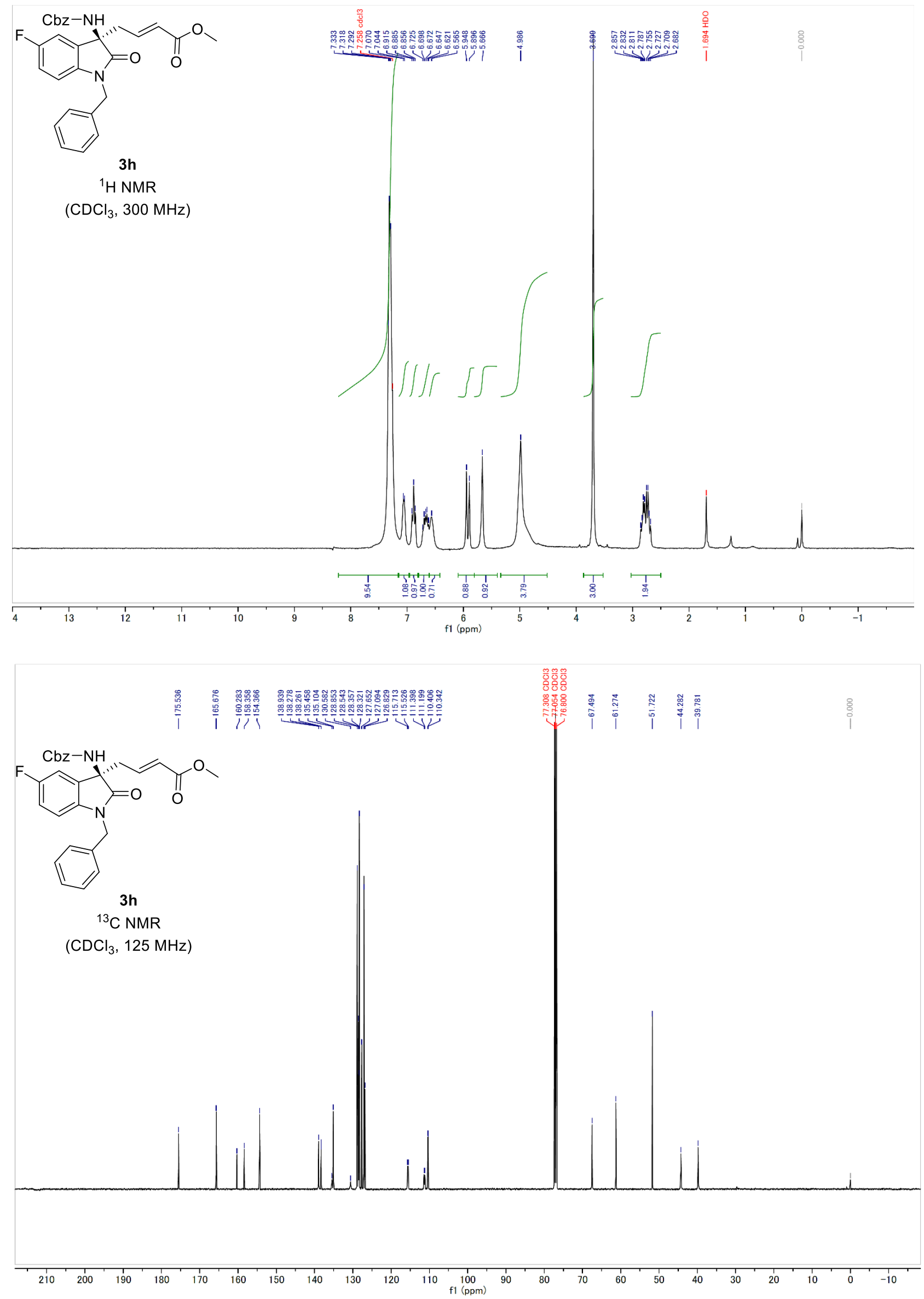


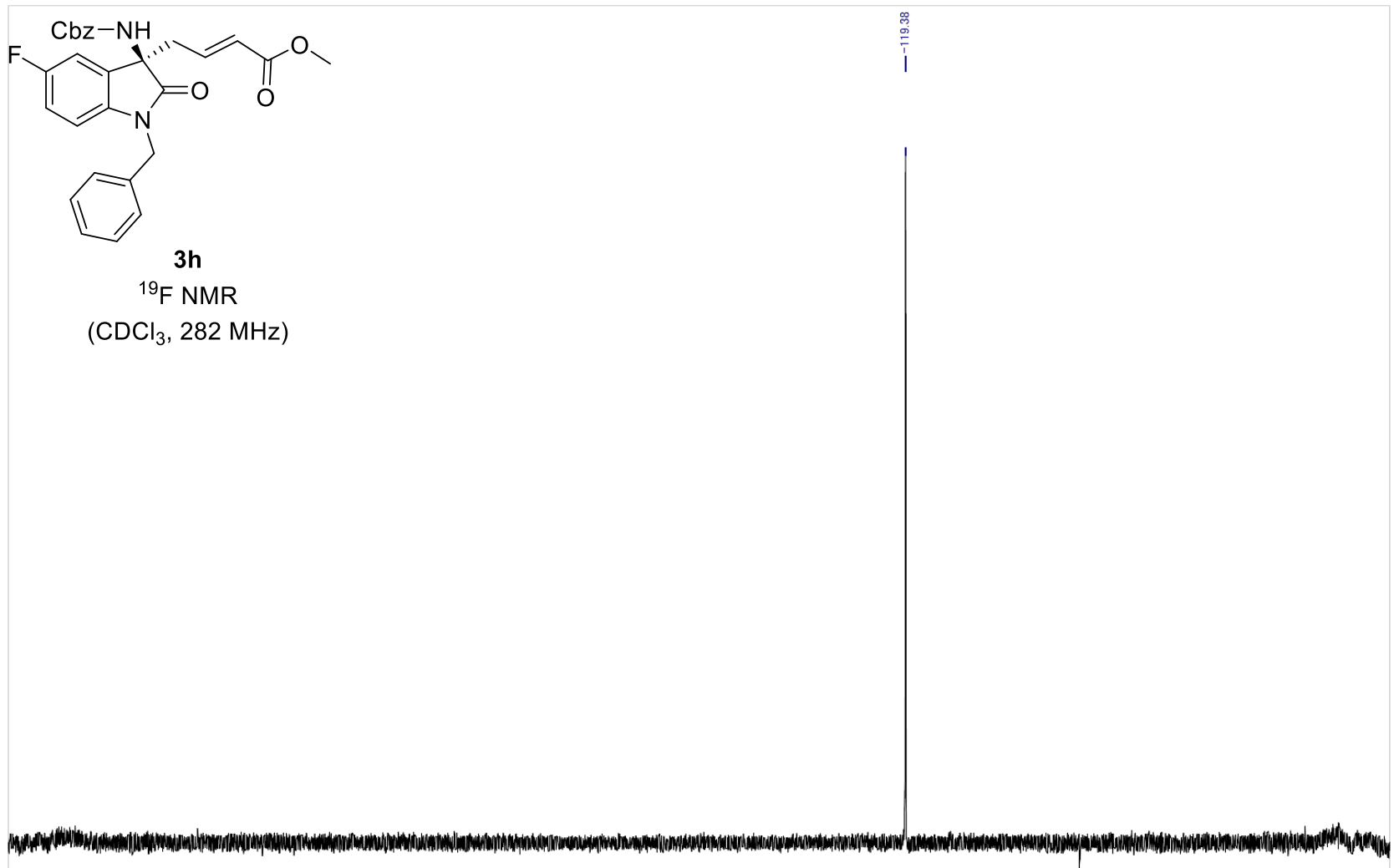

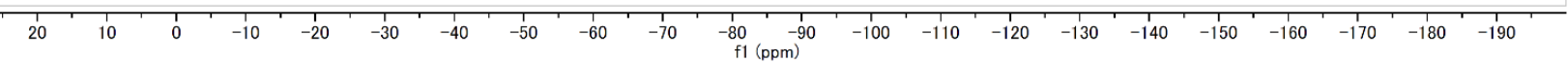



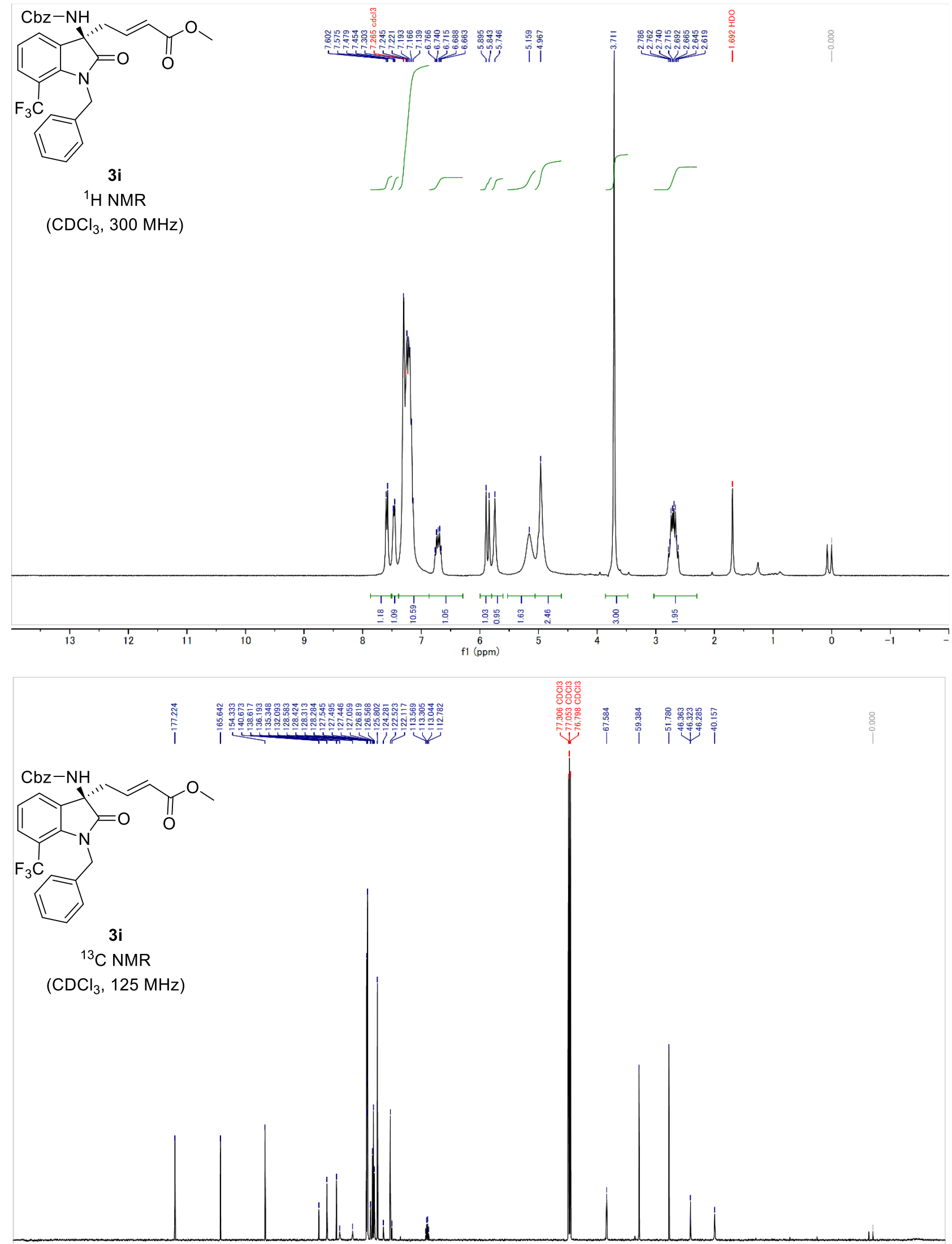

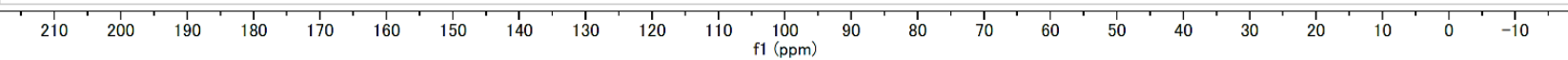




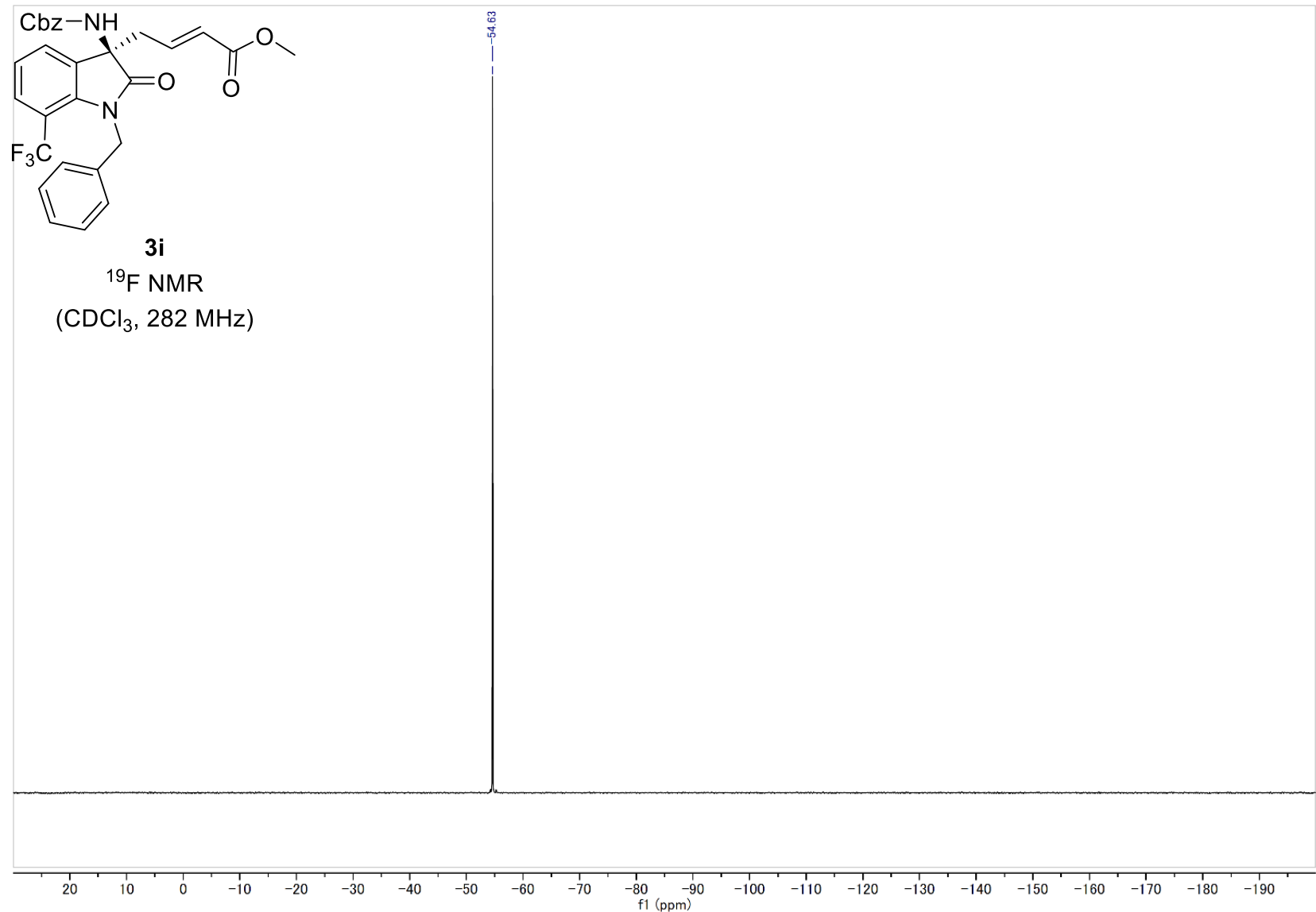



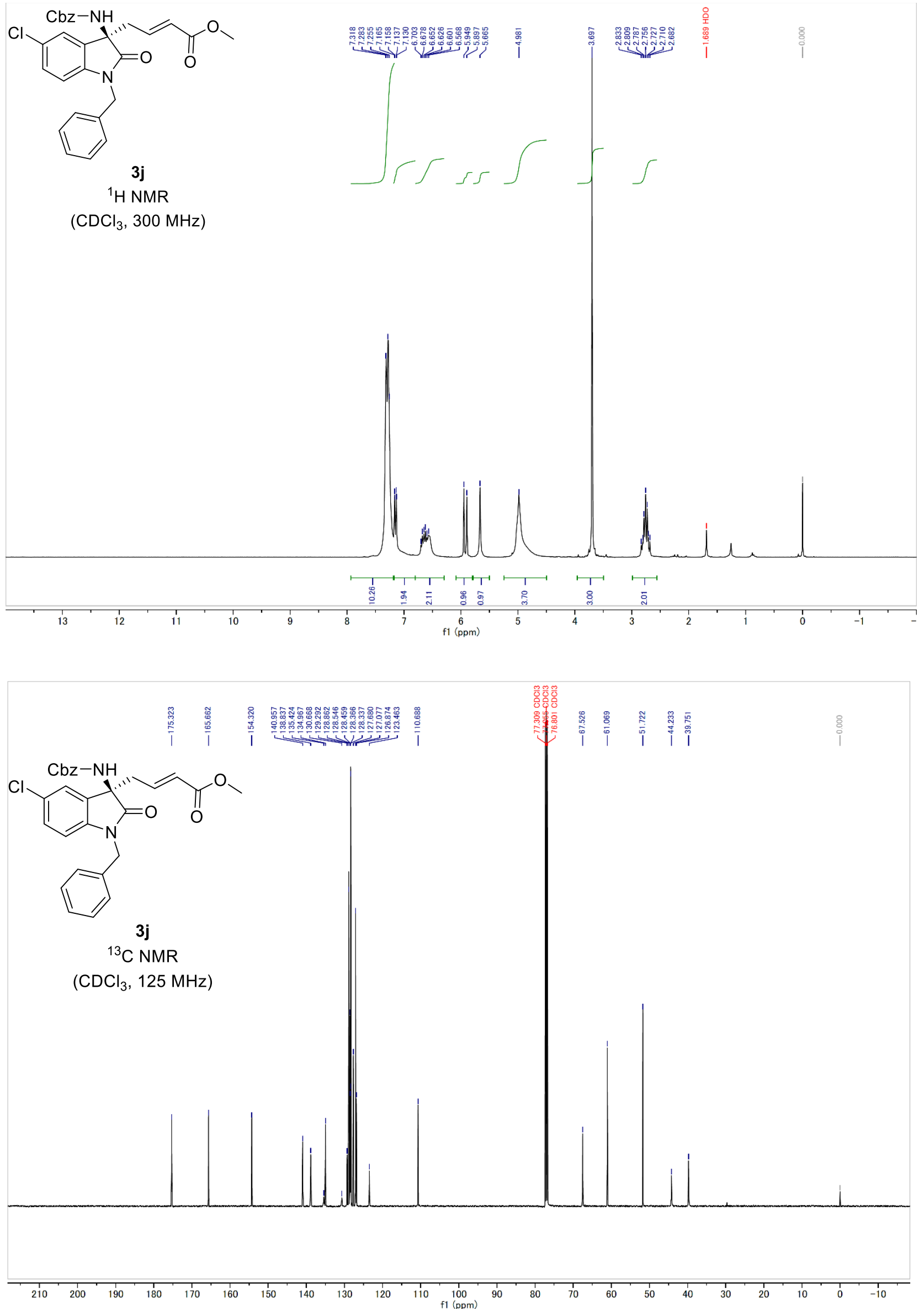

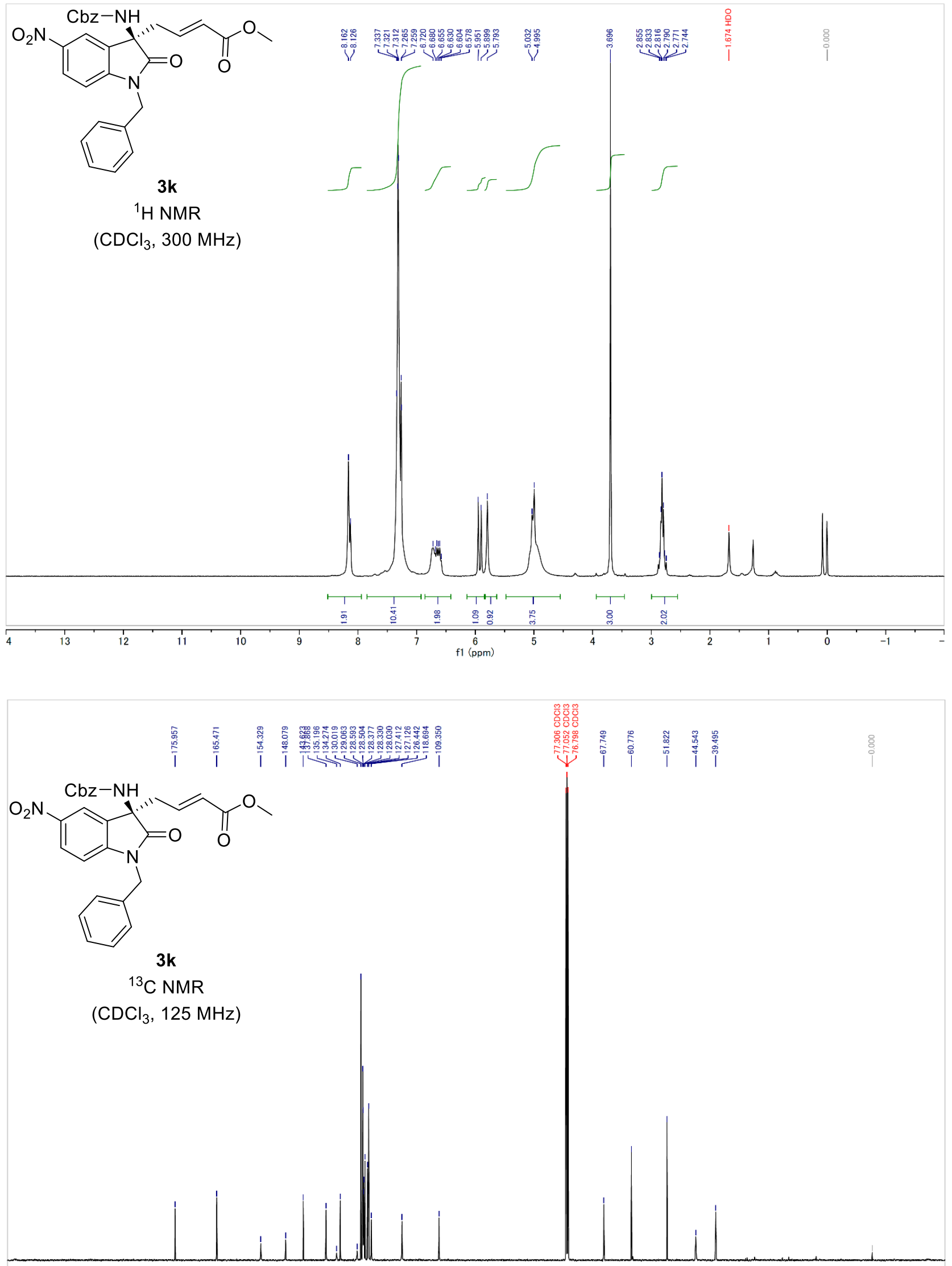

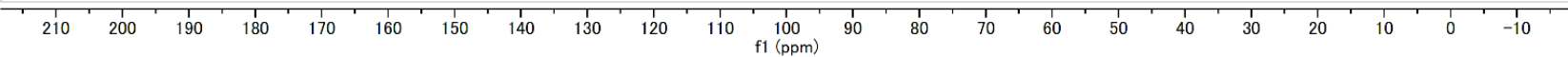



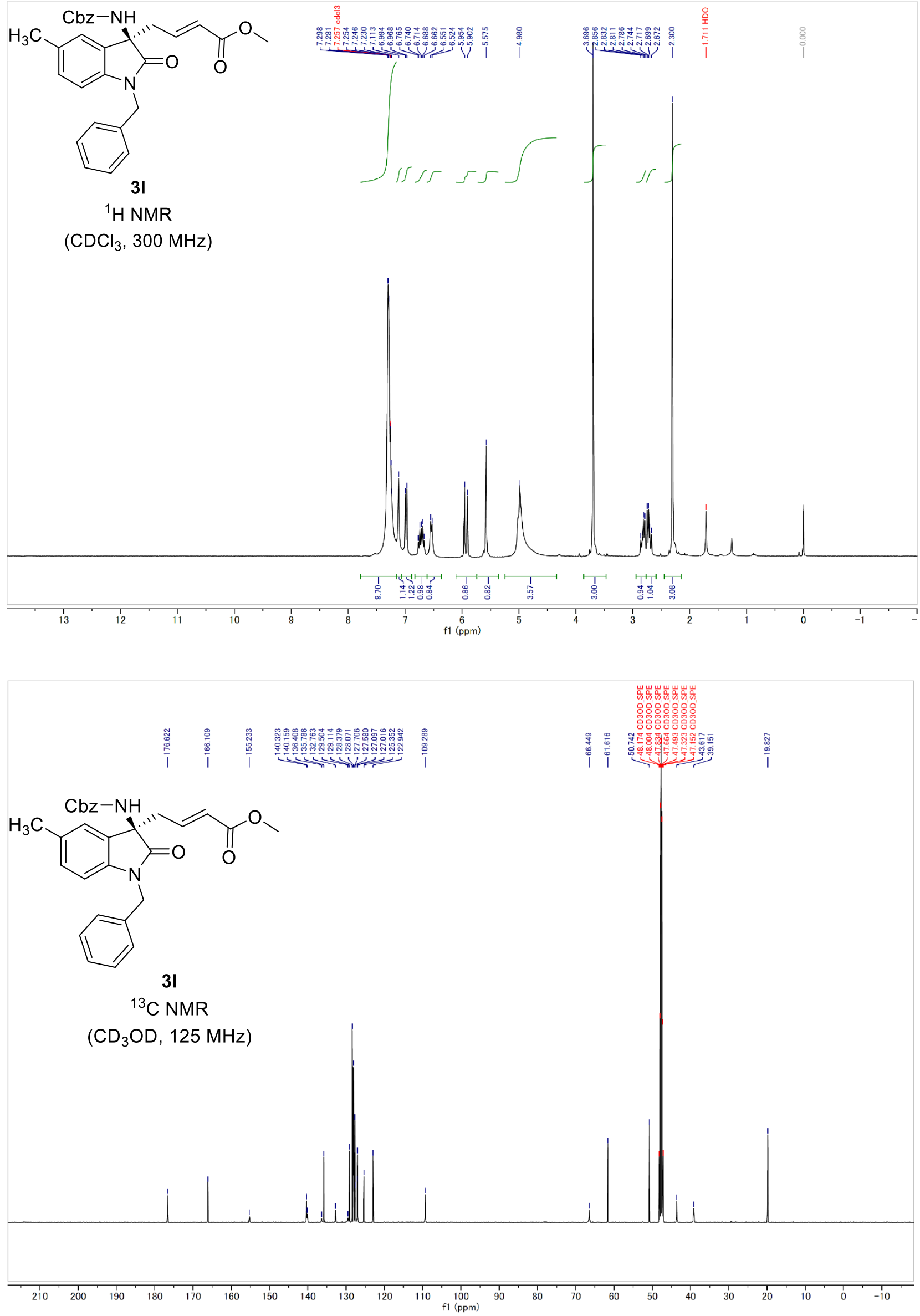

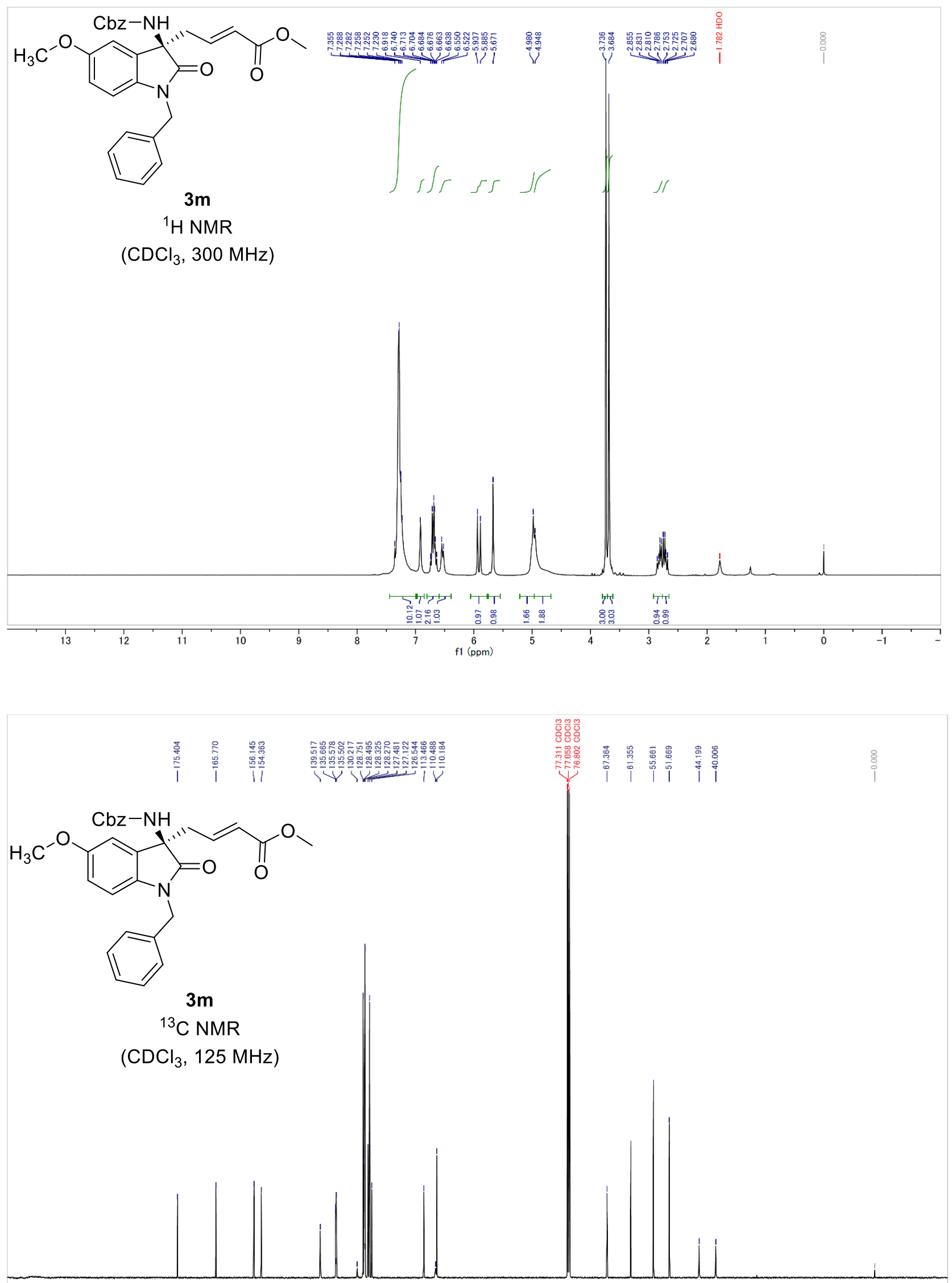

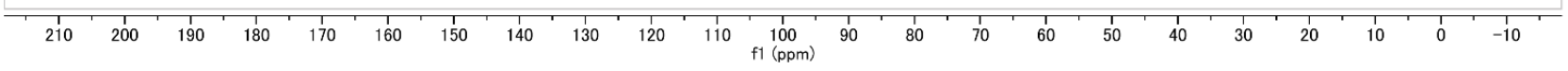



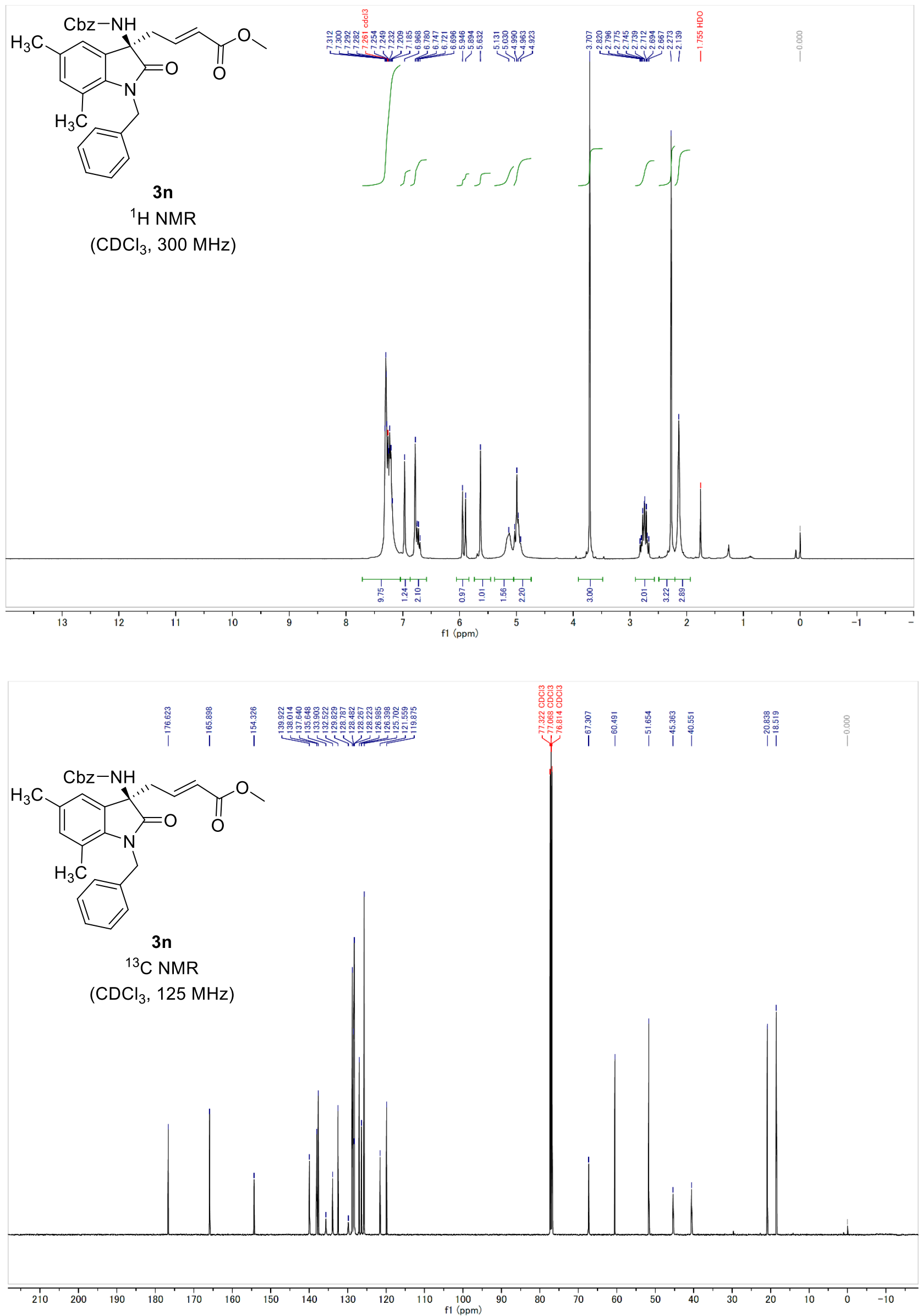

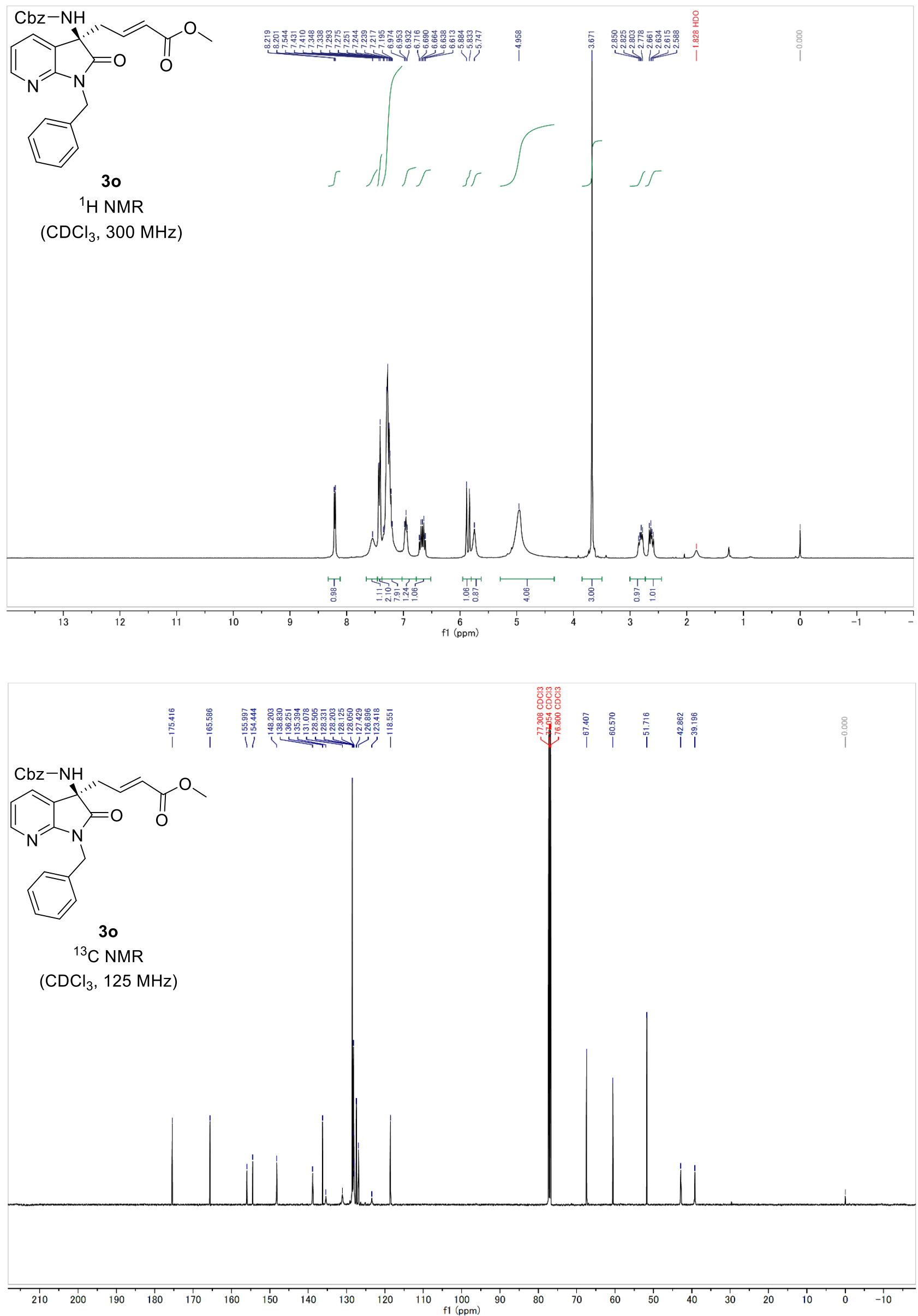

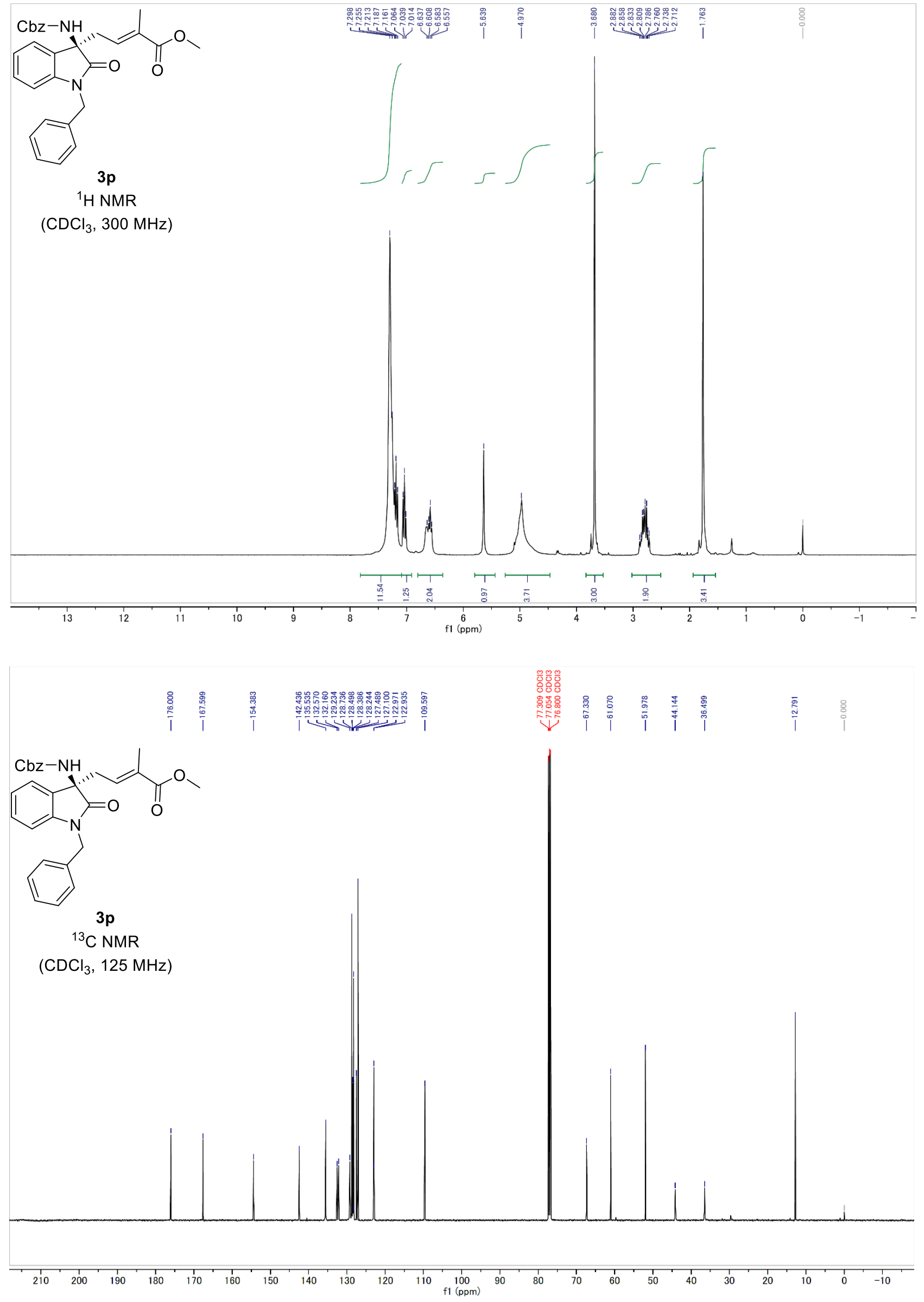

S43 

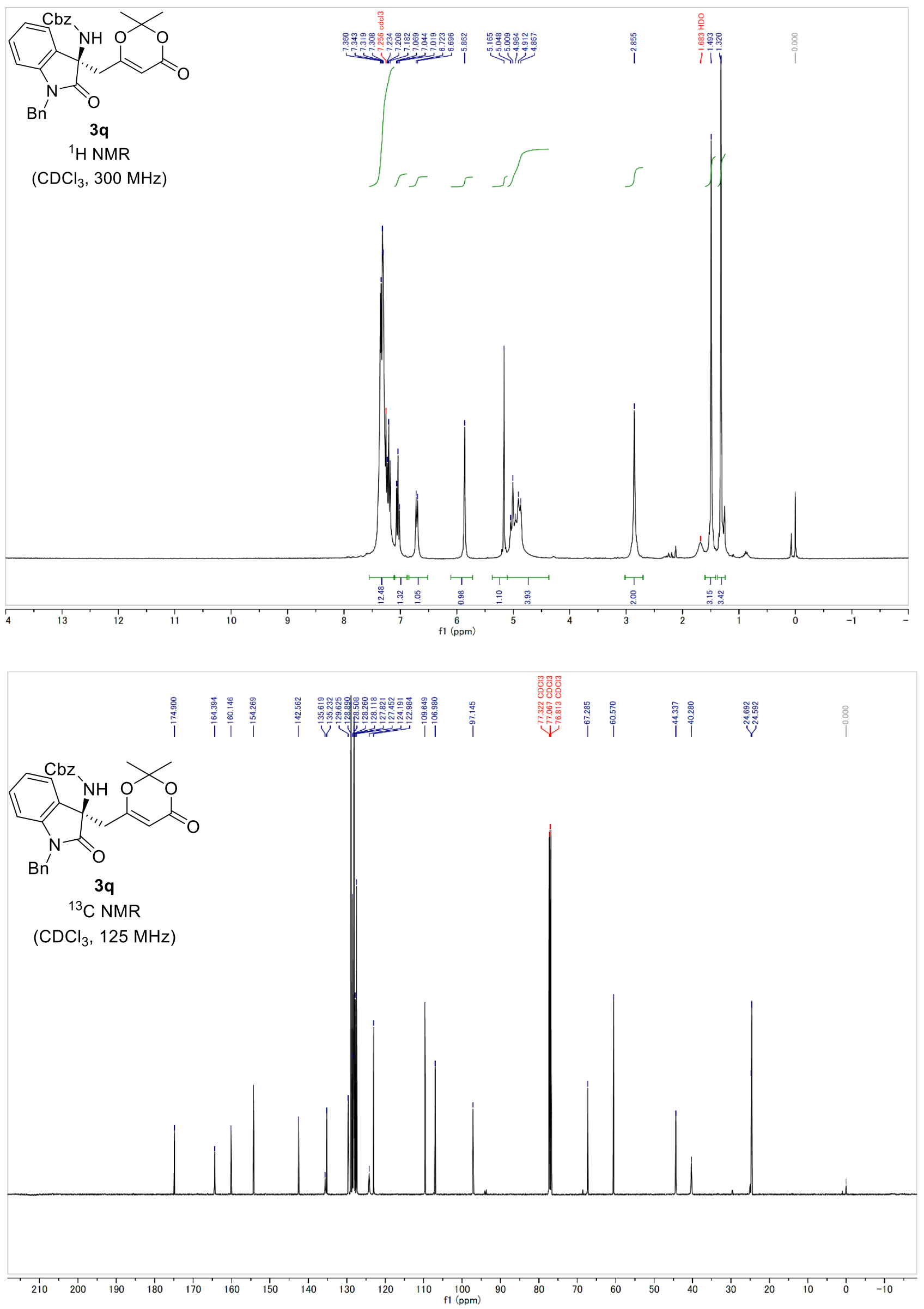

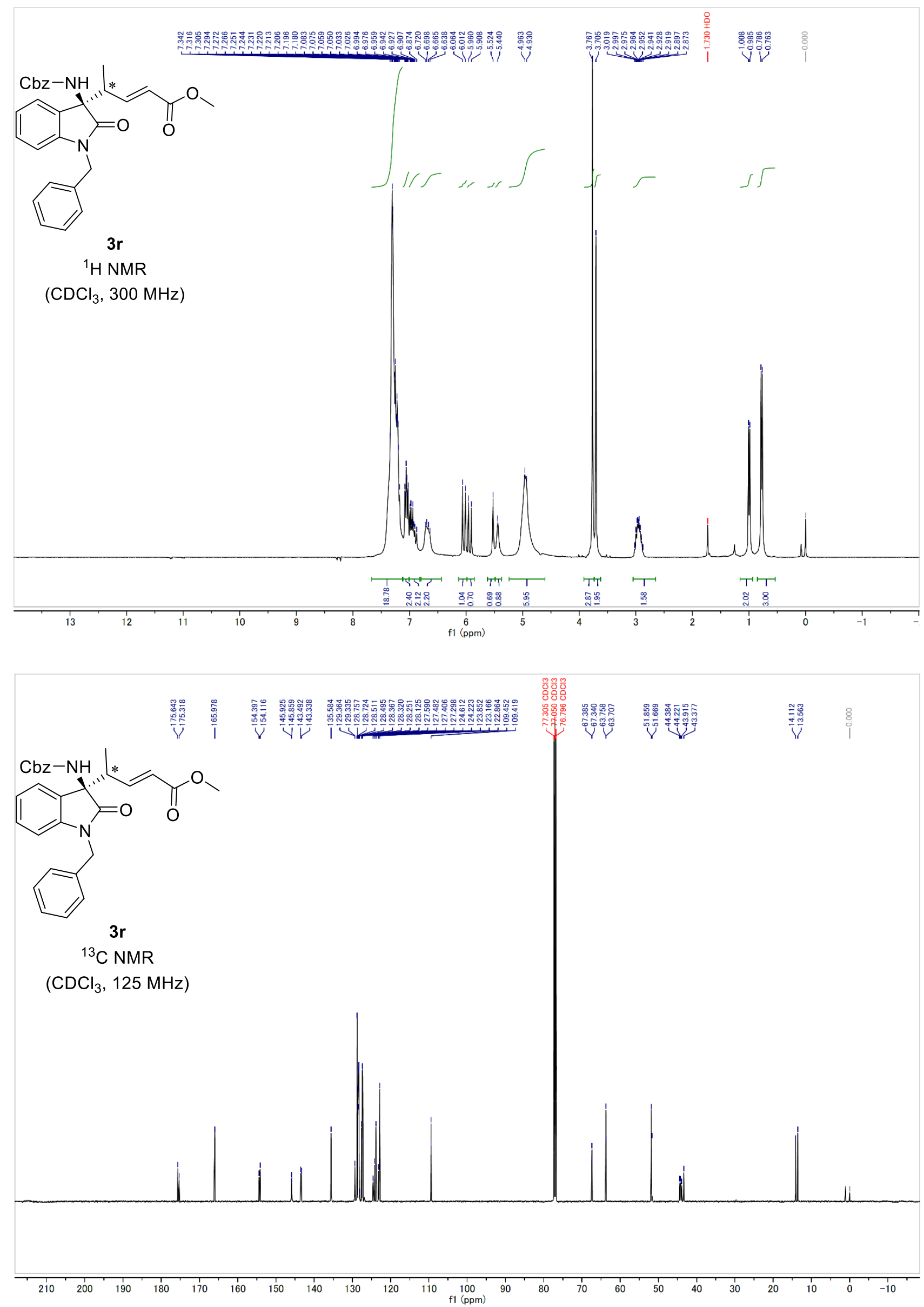

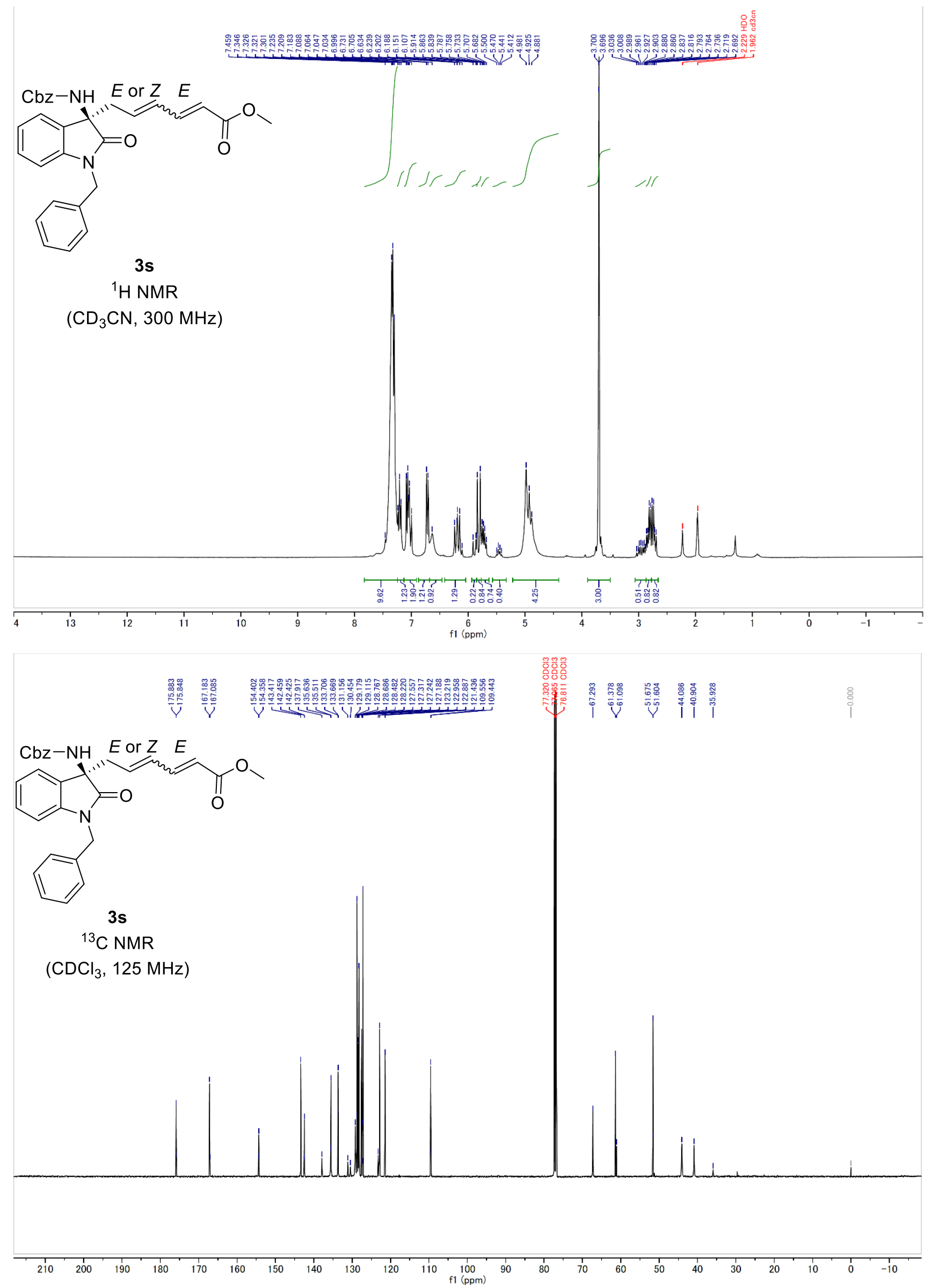

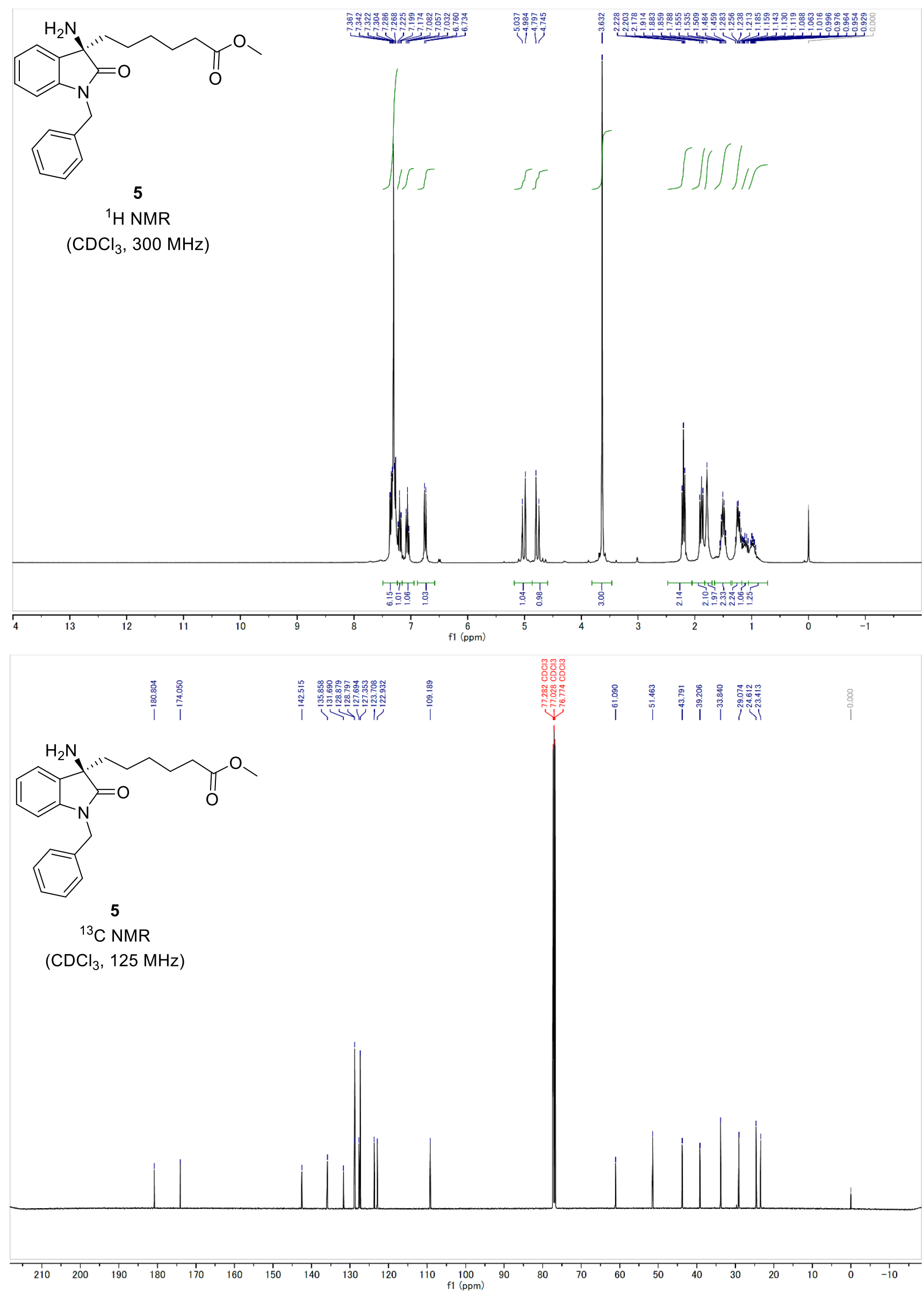

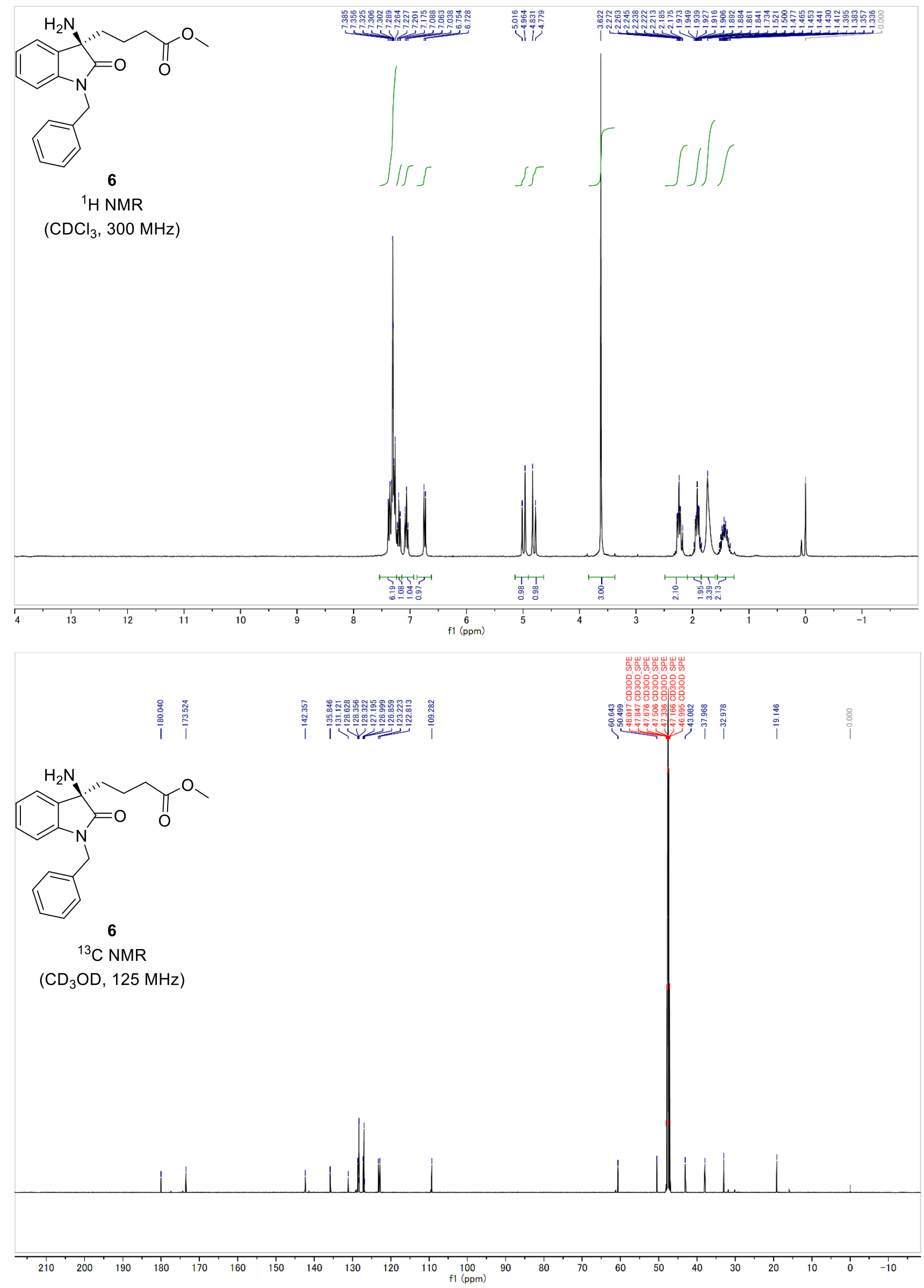

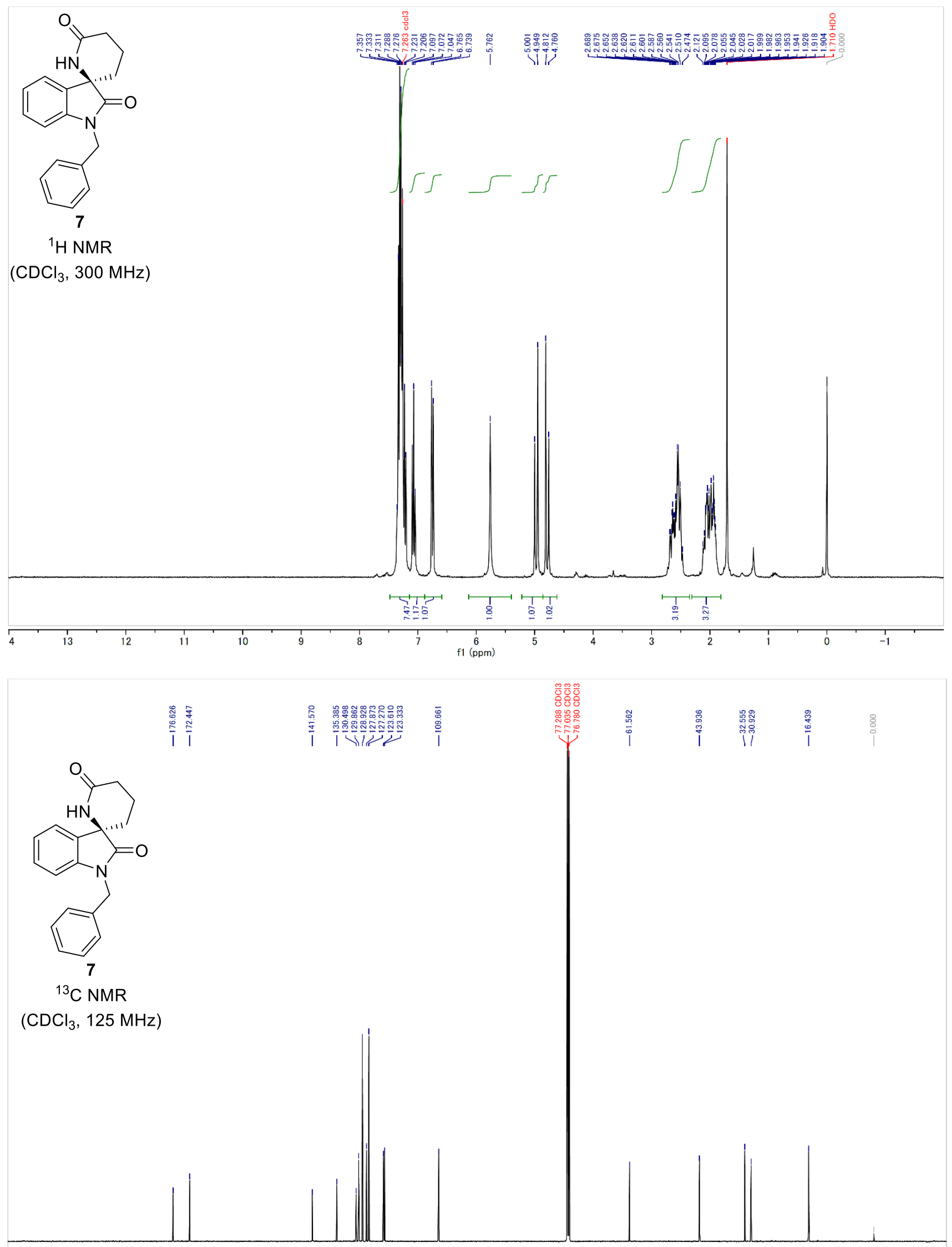

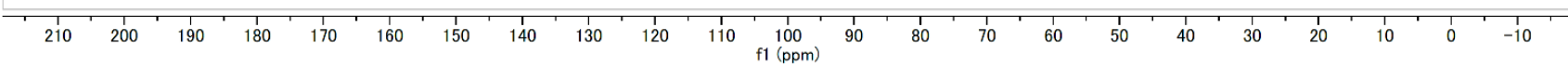



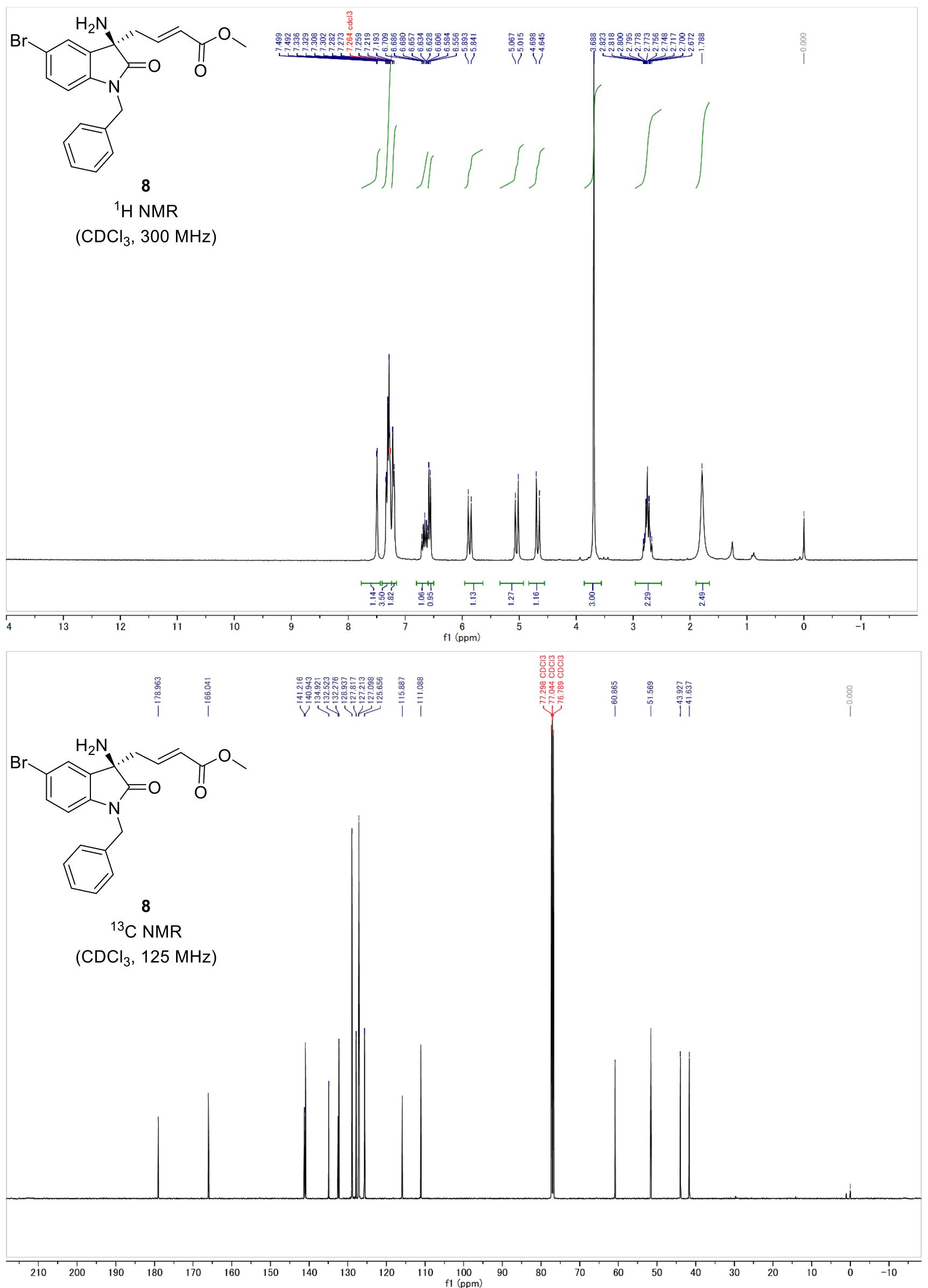


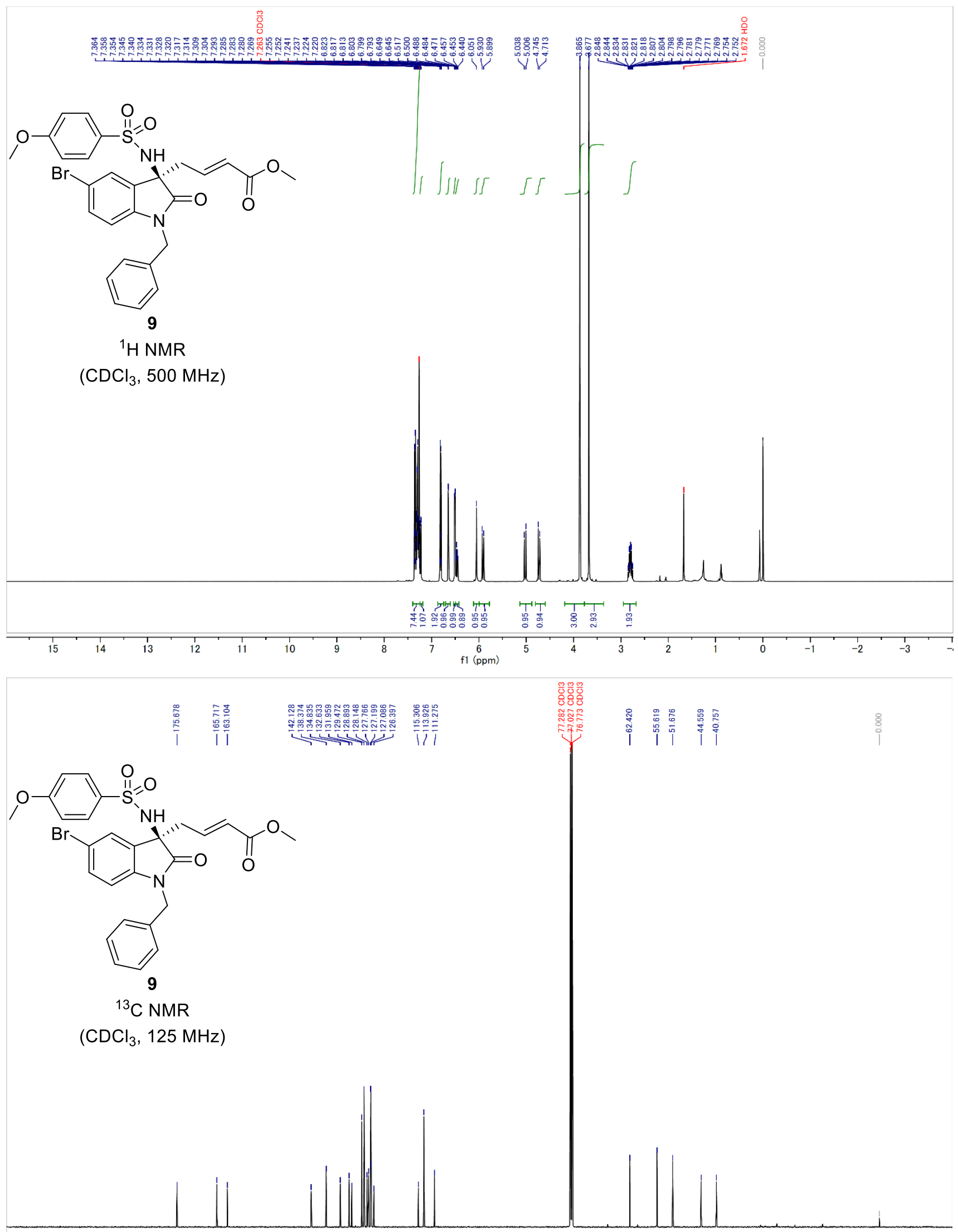

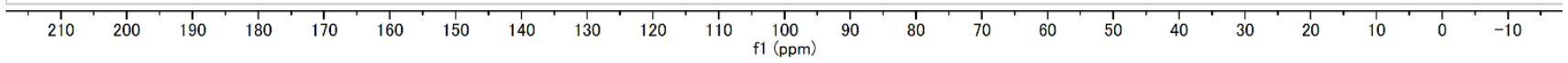




\section{HPLC analysis}

Methyl $(R, E)-4-\{3-[(t e r t-b u t o x y c a r b o n y l) a m i n o]-2-o x o i n d o l i n-3-y l\} b u t-2-e n o a t e(3 a)$<smiles>COC(=O)/C=C/CN[C@@]1(NC(=O)c2ccccc2)C(=O)Nc2ccccc21</smiles>

racemic-3a

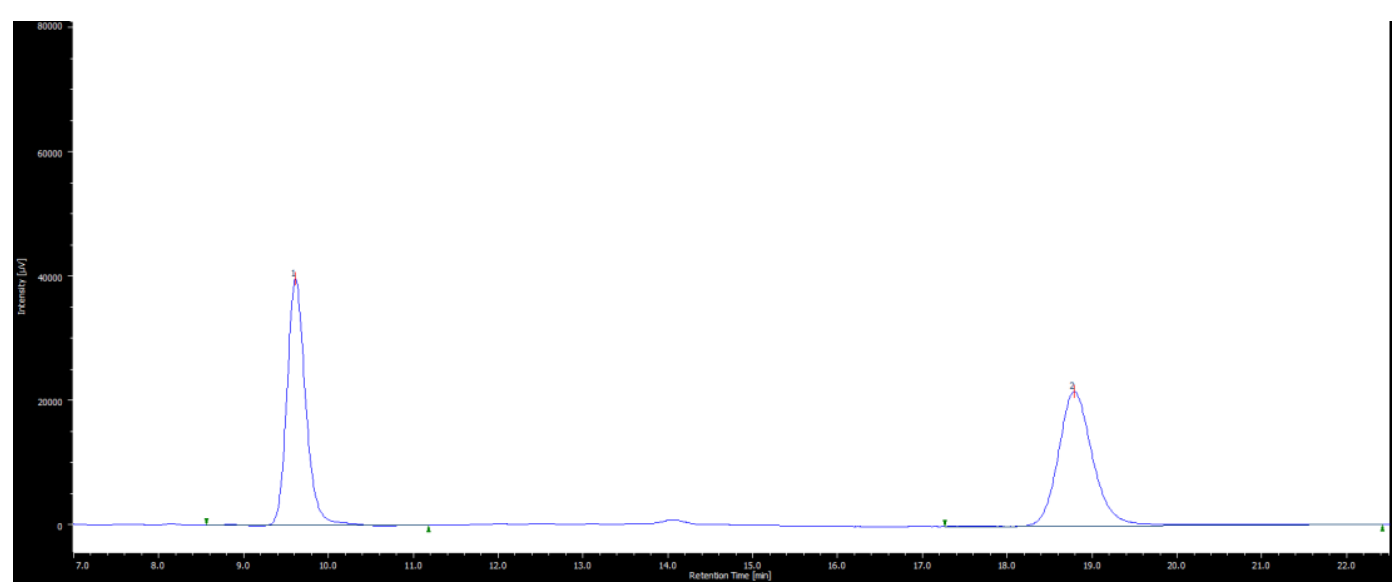

$(R)-\mathbf{3 a}$

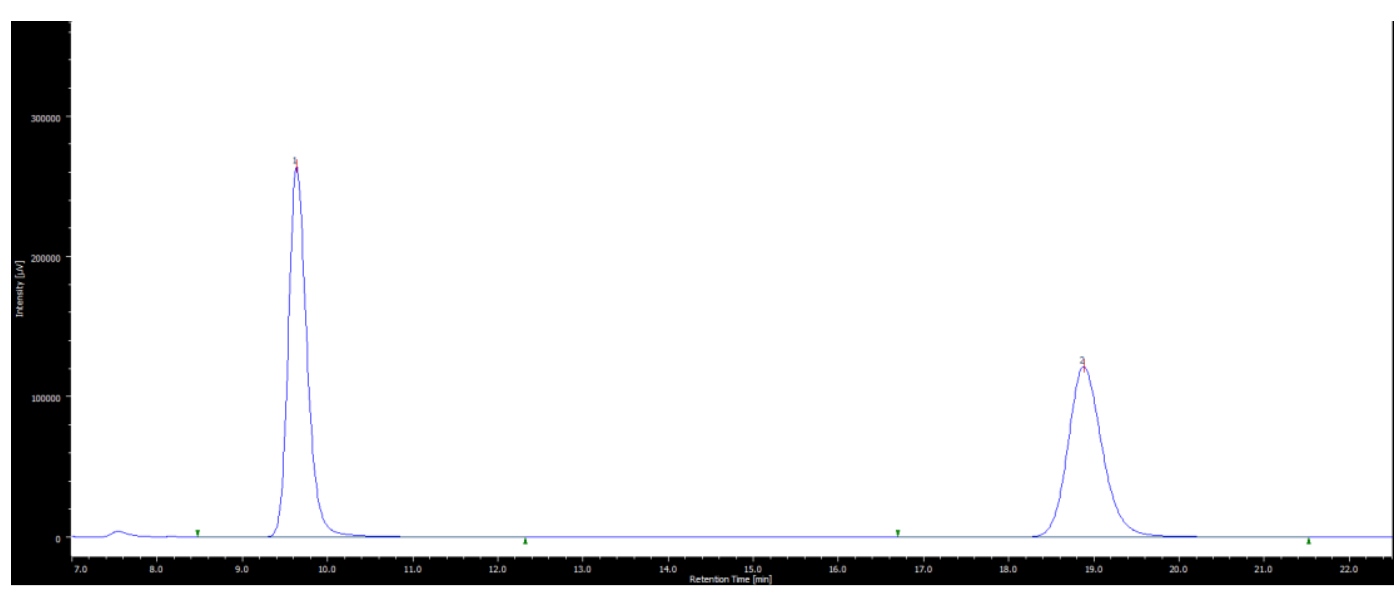

\begin{tabular}{|c|c|c|}
\hline \multicolumn{3}{|c|}{ racemic-3a } \\
\hline Peak & tR (min) & Area (\%) \\
\hline 1 & 9.6 & 49.8 \\
\hline 2 & 18.8 & 50.0 \\
\hline
\end{tabular}

\begin{tabular}{|c|c|c|}
\hline \multicolumn{3}{|c|}{$(R)-3 a$} \\
\hline Peak & tR (min) & Area (\%) \\
\hline 1 & 9.6 & 54.0 \\
\hline 2 & 18.9 & 46.0 \\
\hline
\end{tabular}


Methyl (R,E)-4-[3-(benzyloxycarbonyl)amino-2-oxoindolin-3-yl]but-2-enoate (3b)<smiles>COC(=O)/C=C/C[C@]1(NC(=O)OCc2ccccc2)C(=O)Nc2ccccc21</smiles>

racemic-3b

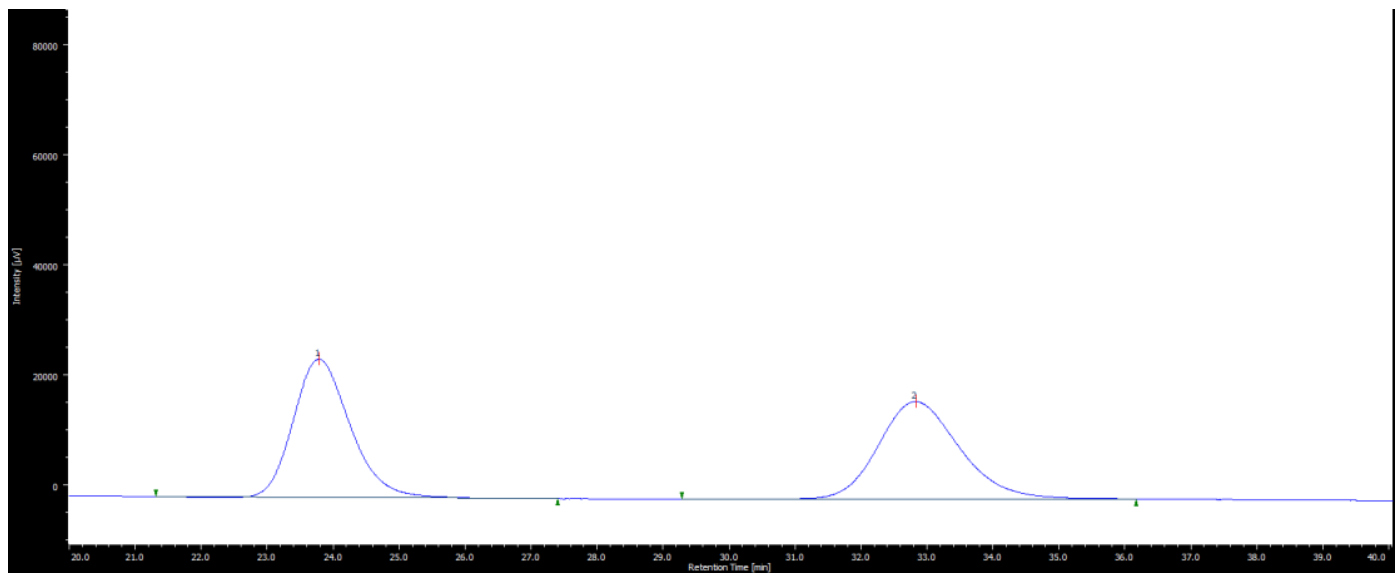

(R)-3b

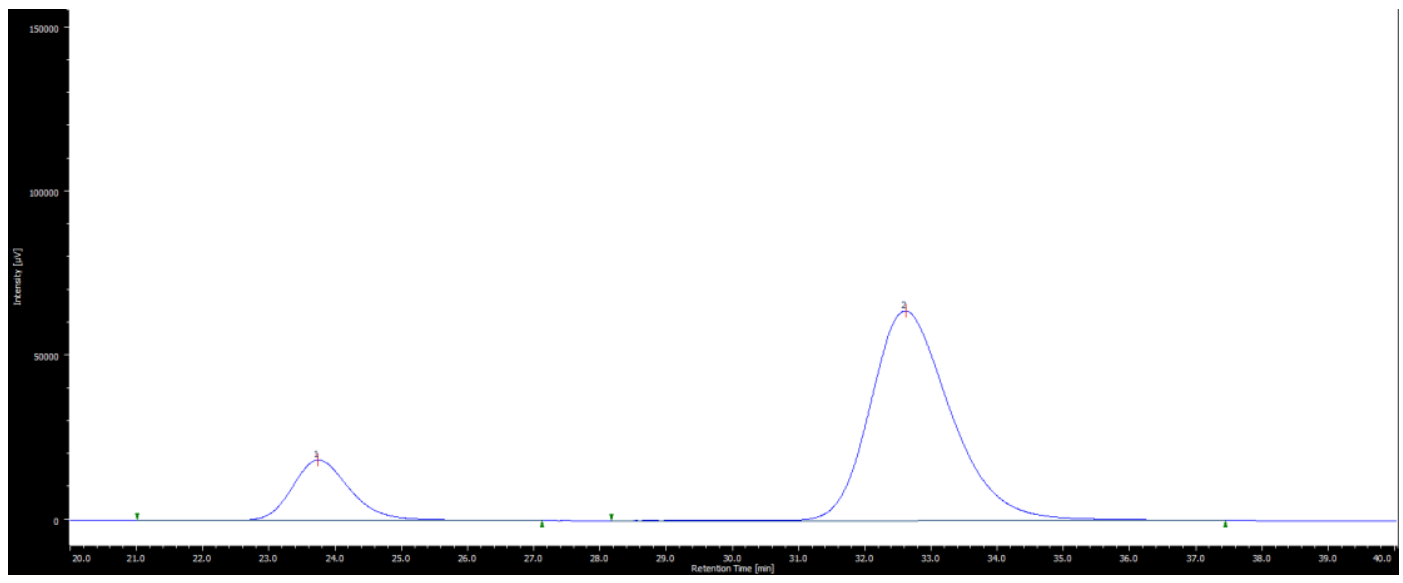

\begin{tabular}{|c|c|c|}
\hline \multicolumn{3}{|c|}{ racemic-3b } \\
\hline Peak & tR (min) & Area (\%) \\
\hline 1 & 23.8 & 49.9 \\
\hline 2 & 32.8 & 50.1 \\
\hline
\end{tabular}

\begin{tabular}{|c|c|c|}
\hline \multicolumn{3}{|c|}{$(R)-3 b$} \\
\hline Peak & tR (min) & Area (\%) \\
\hline 1 & 23.7 & 17.0 \\
\hline 2 & 32.6 & 83.0 \\
\hline
\end{tabular}


Methyl (R,E)-4-[1-methyl-3-(benzyloxycarbonylamino)-2-oxoindolin-3-yl]but-2-enoate (3c)<smiles>COC(=O)/C=C/C[C@@]1(NC(=O)OCc2ccccc2)C(=O)N(C)c2ccccc21</smiles>

racemic-3c

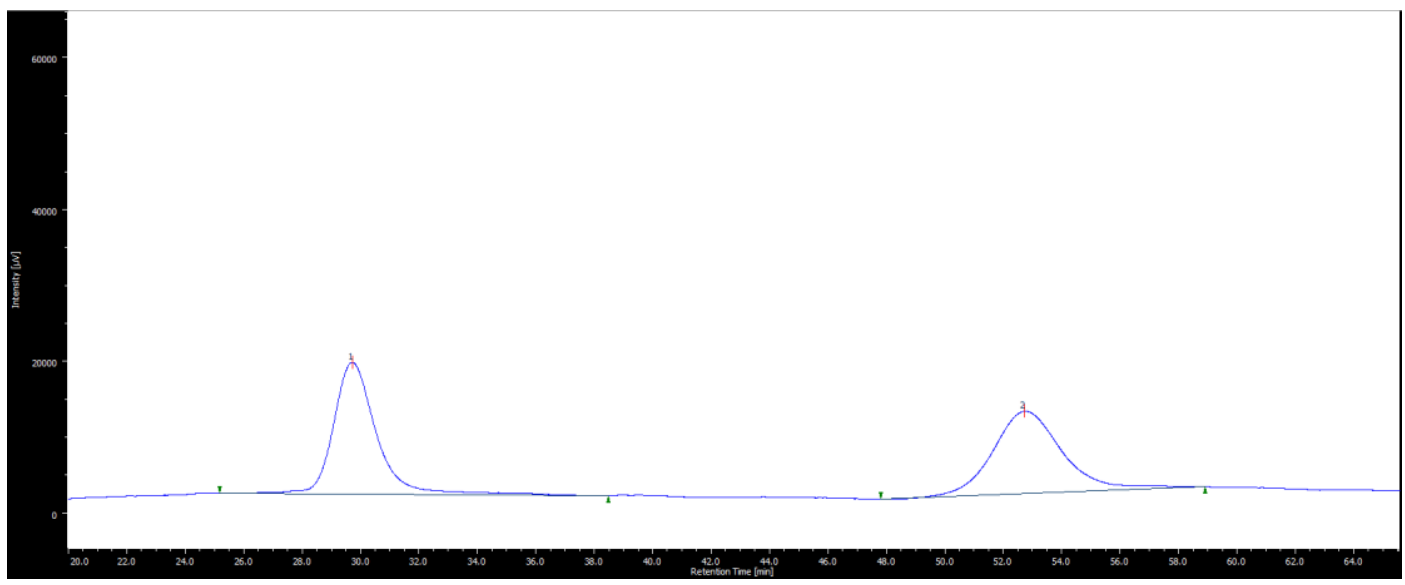

(R)-3c

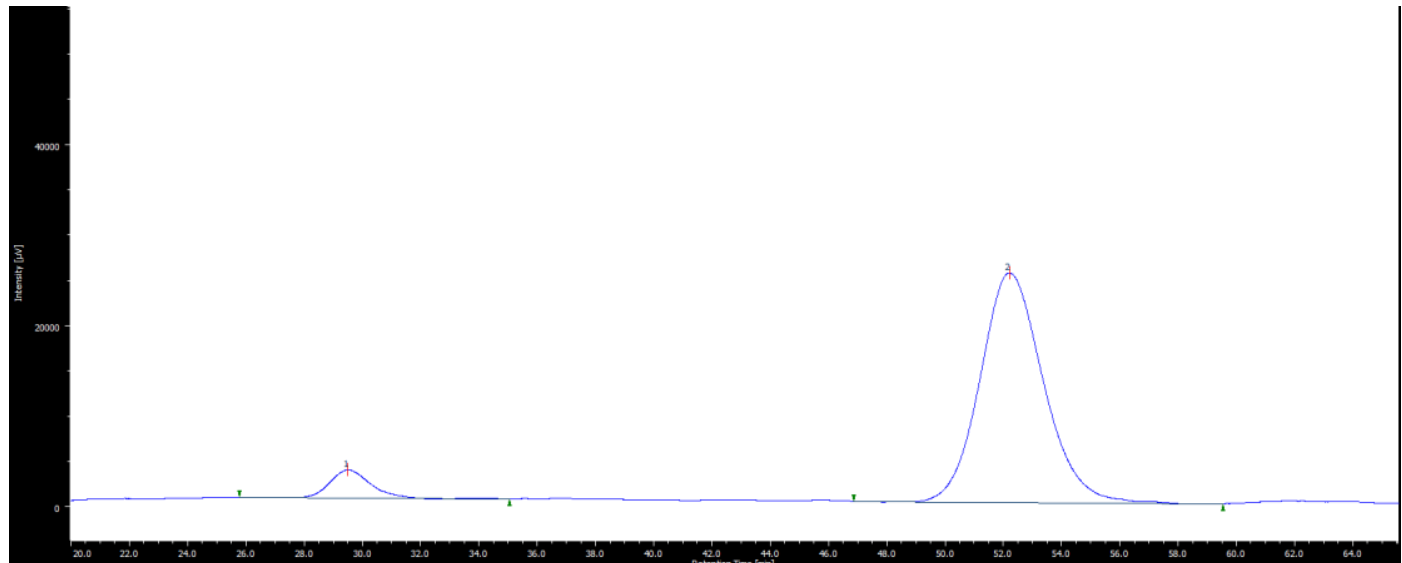

\begin{tabular}{|c|c|c|}
\hline \multicolumn{3}{|c|}{ racemic-3c } \\
\hline Peak & tR (min) & Area (\%) \\
\hline 1 & 29.7 & 49.8 \\
\hline 2 & 52.7 & 50.2 \\
\hline
\end{tabular}

\begin{tabular}{|c|c|c|}
\hline \multicolumn{3}{|c|}{$(R)-3 c$} \\
\hline Peak & tR (min) & Area (\%) \\
\hline 1 & 29.5 & 7.3 \\
\hline 2 & 52.2 & 92.7 \\
\hline
\end{tabular}


Methyl (R,E)-4-[1-benzyl-3-(benzyloxycarbonylamino)-2-oxoindolin-3-yl]but-2-enoate (3d)

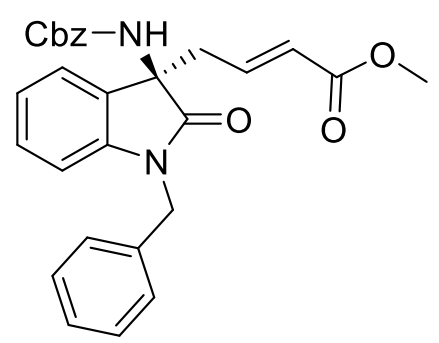

racemic-3d

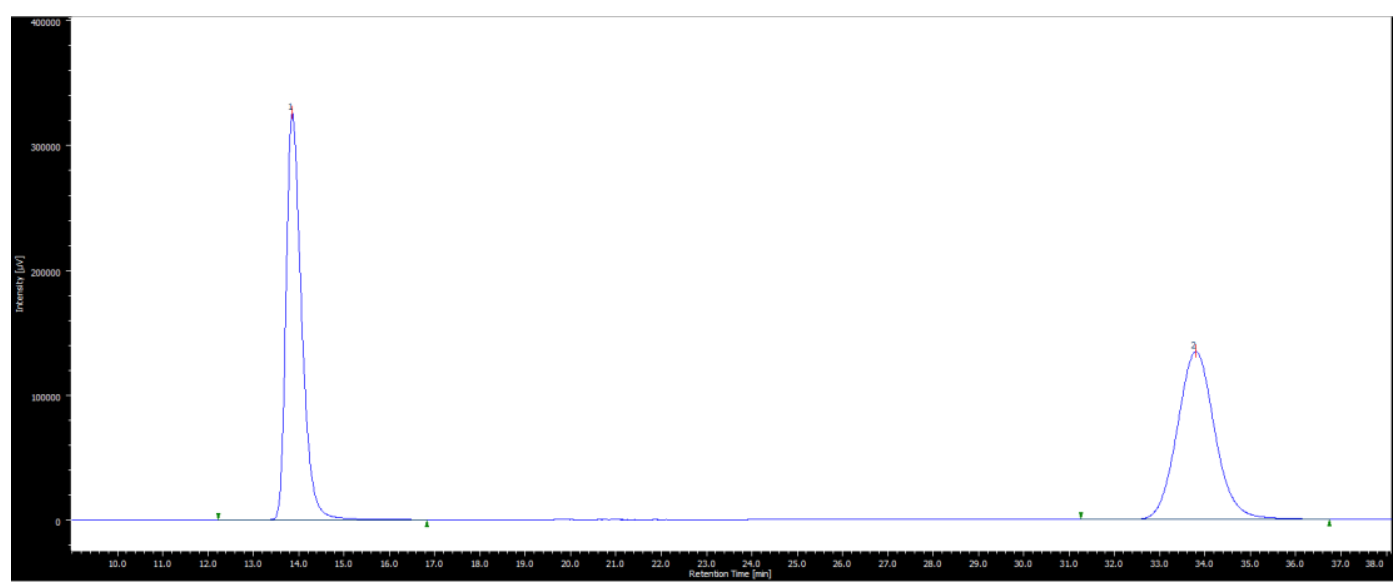

(R)-3d

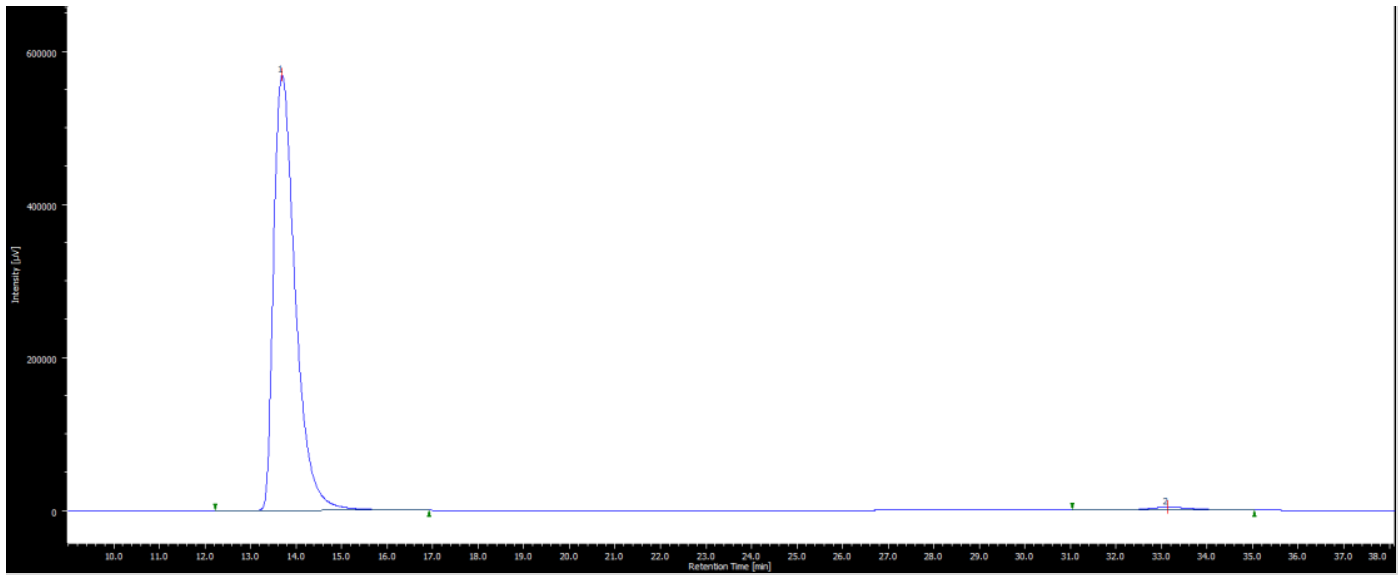

\begin{tabular}{|c|c|c|}
\hline \multicolumn{3}{|c|}{ racemic-3d } \\
\hline Peak & tR (min) & Area (\%) \\
\hline 1 & 13.9 & 50.0 \\
\hline 2 & 33.8 & 50.0 \\
\hline
\end{tabular}

\begin{tabular}{|c|c|c|}
\hline \multicolumn{3}{|c|}{$(R)-3 d$} \\
\hline Peak & tR (min) & Area (\%) \\
\hline 1 & 9.1 & 98.6 \\
\hline 2 & 33.1 & 1.4 \\
\hline
\end{tabular}


Methyl (R,E)-4-[1-benzyl-3-(benzyloxycarbonylamino)-5-bromo-2-oxoindolin-3-yl]but-2-enoate (3e)

racemic-3e
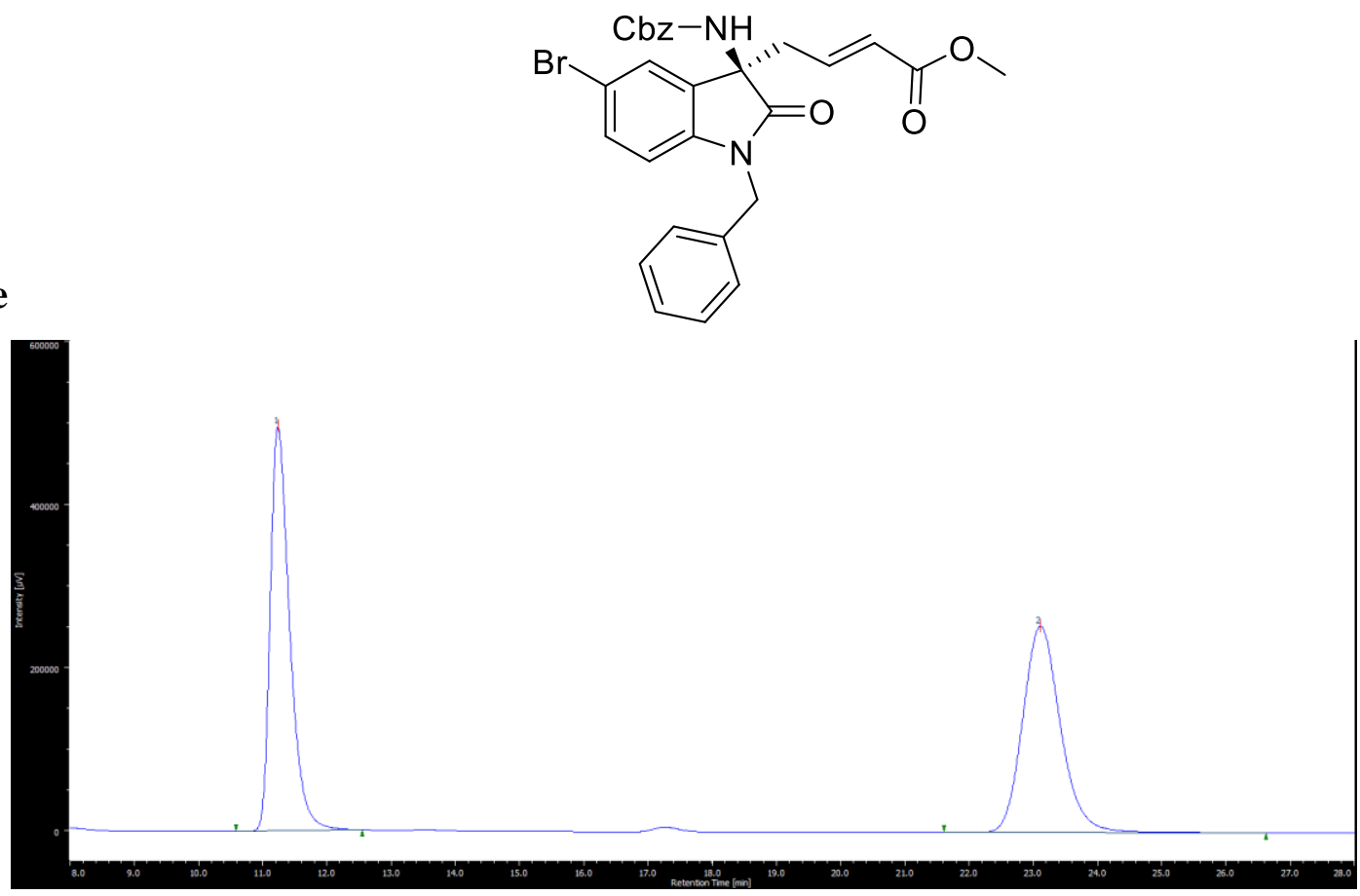

$(R)-3 \mathbf{e}$

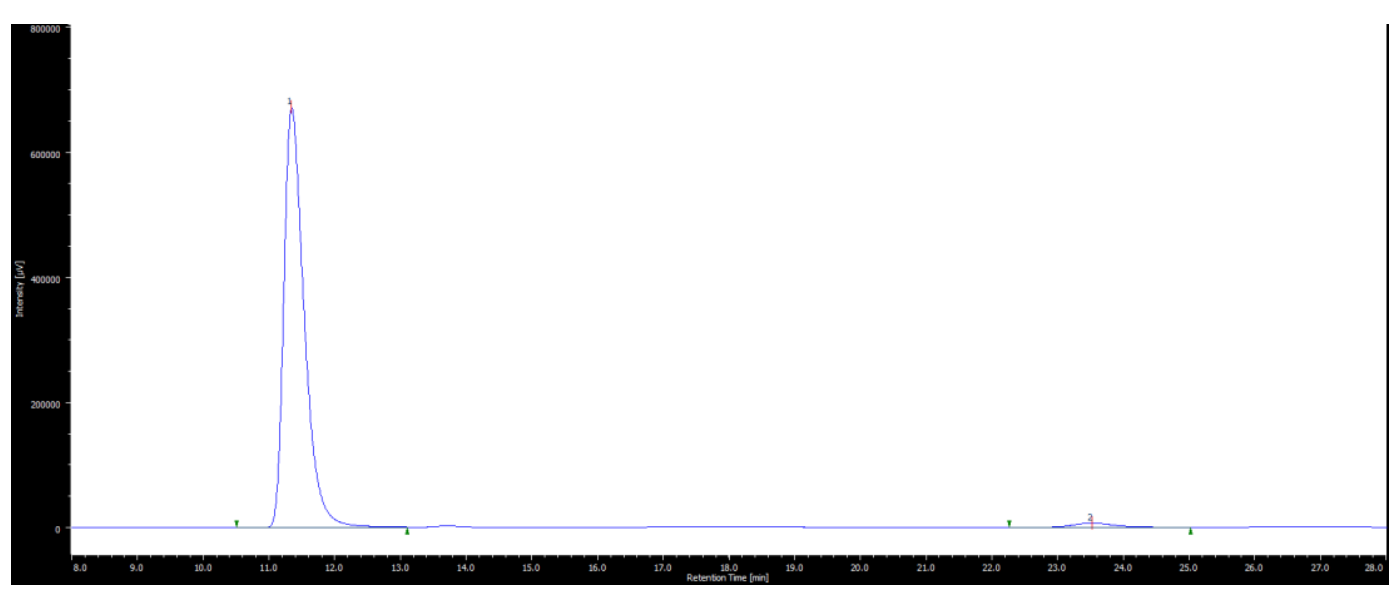

\begin{tabular}{|c|c|c|}
\hline \multicolumn{3}{|c|}{ racemic-3e } \\
\hline Peak & tR (min) & Area (\%) \\
\hline 1 & 11.2 & 50.1 \\
\hline 2 & 23.1 & 49.9 \\
\hline
\end{tabular}

\begin{tabular}{|c|c|c|}
\hline \multicolumn{3}{|c|}{$(R)-3 \mathrm{e}$} \\
\hline Peak & tR (min) & Area (\%) \\
\hline 1 & 11.3 & 98.0 \\
\hline 2 & 23.5 & 2.0 \\
\hline
\end{tabular}


Methyl (R,E)-4-[1-benzyl-3-(benzyloxycarbonylamino)-6-bromo-2-oxoindolin-3-yl]but-2-enoate (3f)

racemic-3f<smiles>COC(=O)/C=C/C[C@@]1(NC(=O)O)C(=O)N(Cc2ccccc2)c2cc(Br)ccc21</smiles>

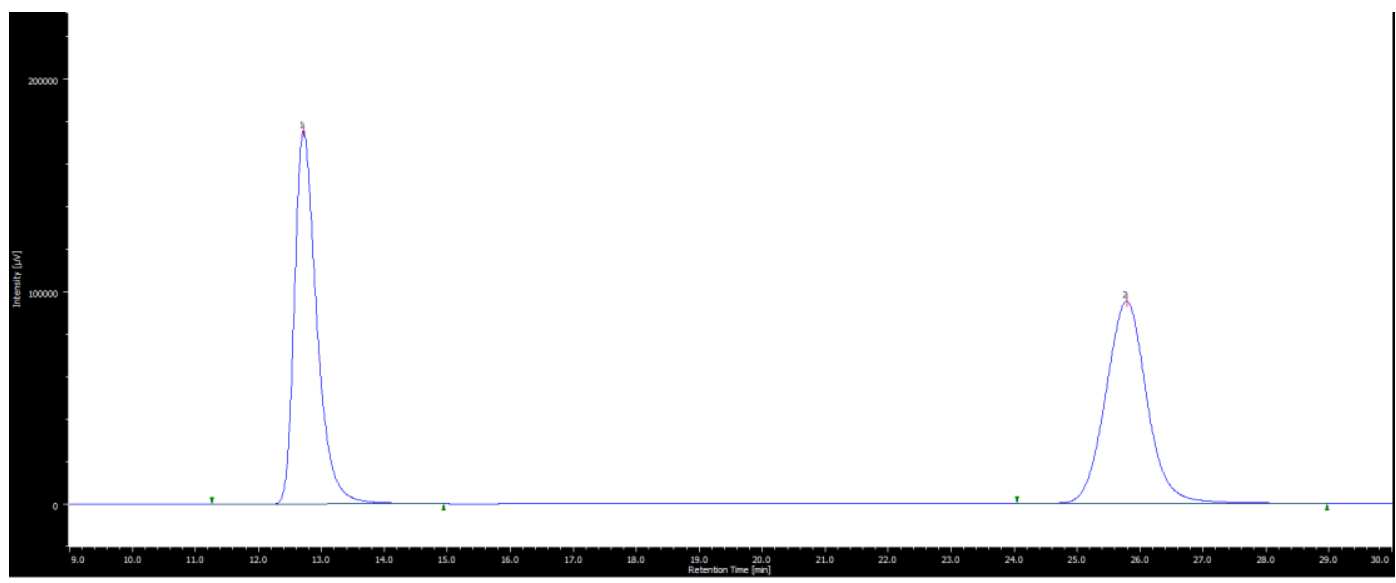

(R)-3f

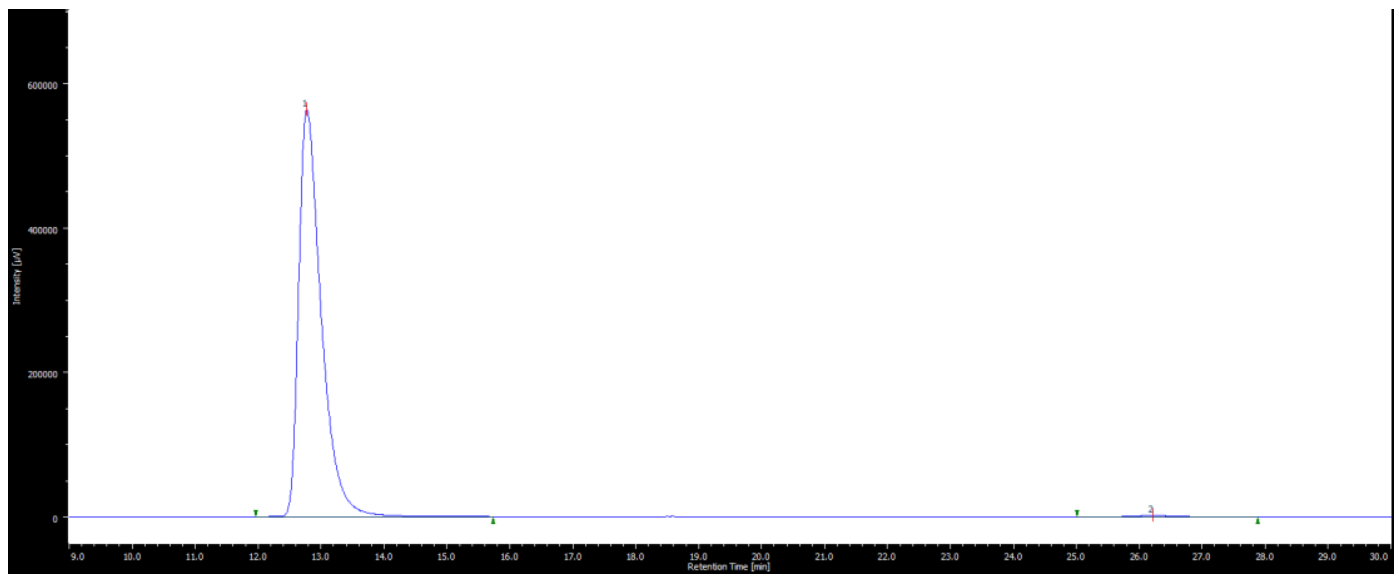

\begin{tabular}{|c|c|c|}
\hline \multicolumn{3}{|c|}{ racemic-3f } \\
\hline Peak & tR (min) & Area (\%) \\
\hline 1 & 12.7 & 50.1 \\
\hline 2 & 25.8 & 49.9 \\
\hline
\end{tabular}

\begin{tabular}{|c|c|c|}
\hline \multicolumn{3}{|c|}{$(R)-3 f$} \\
\hline Peak & tR (min) & Area (\%) \\
\hline 1 & 12.8 & 99.3 \\
\hline 2 & 26.2 & 0.7 \\
\hline
\end{tabular}


Methyl (R,E)-4-[1-benzyl-3-(benzyloxycarbonylamino)-7-bromo-2-oxoindolin-3-yl]but-2-enoate (3g)

racemic-3g
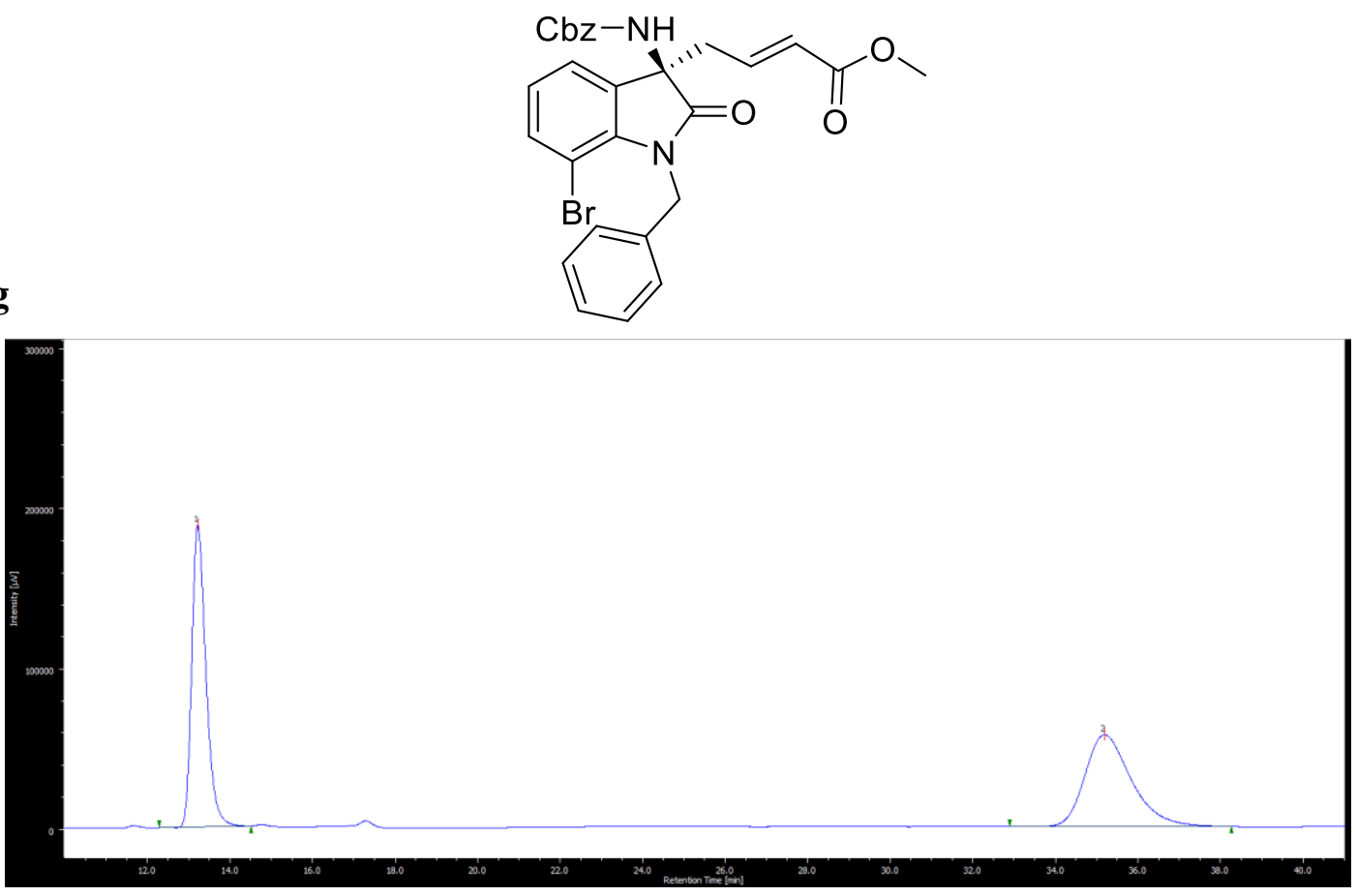

$(R)-3 \mathrm{~g}$

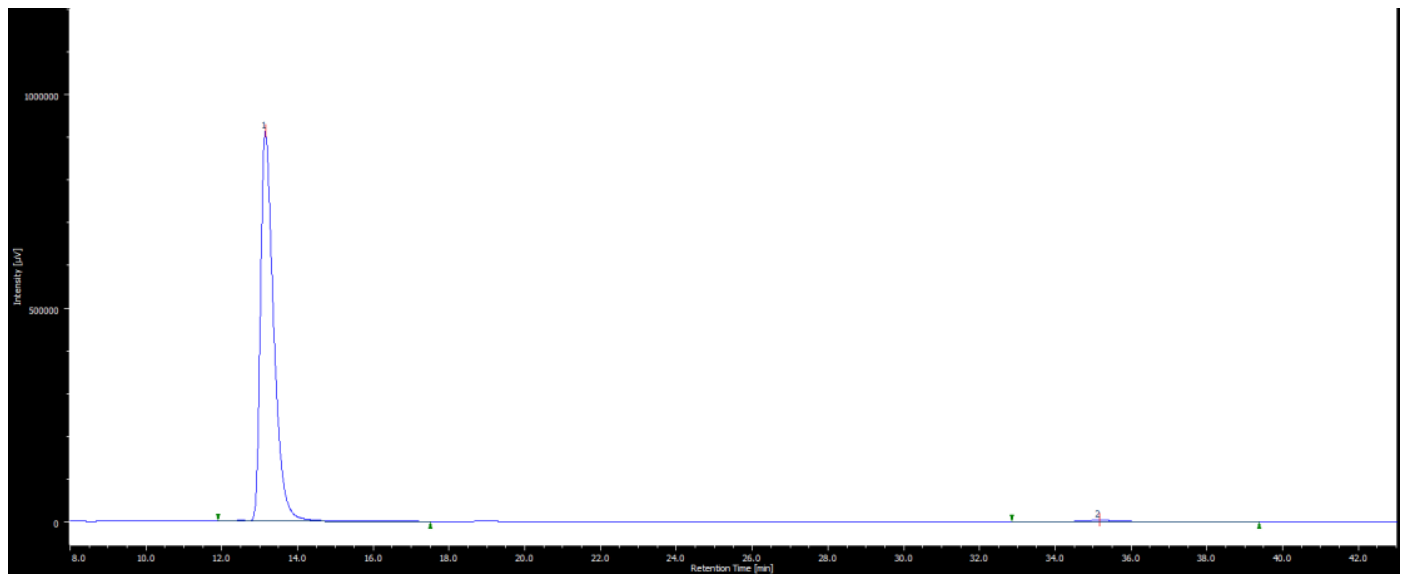

\begin{tabular}{|c|c|c|}
\hline \multicolumn{3}{|c|}{ racemic-3g } \\
\hline Peak & tR (min) & Area (\%) \\
\hline 1 & 13.2 & 50.0 \\
\hline 2 & 35.2 & 50.0 \\
\hline
\end{tabular}

\begin{tabular}{|c|c|c|}
\hline \multicolumn{3}{|c|}{$(R)-3 g$} \\
\hline Peak & tR (min) & Area (\%) \\
\hline 1 & 13.1 & 98.7 \\
\hline 2 & 35.1 & 1.3 \\
\hline
\end{tabular}


Methyl (R,E)-4-[1-benzyl-3-(benzyloxycarbonylamino)-5-fluoro-2-oxoindolin-3-yl]but-2-enoate (3h)

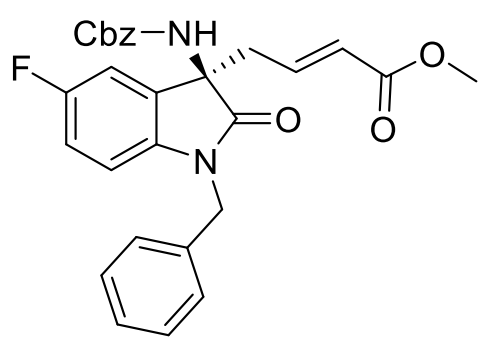

racemic-3h

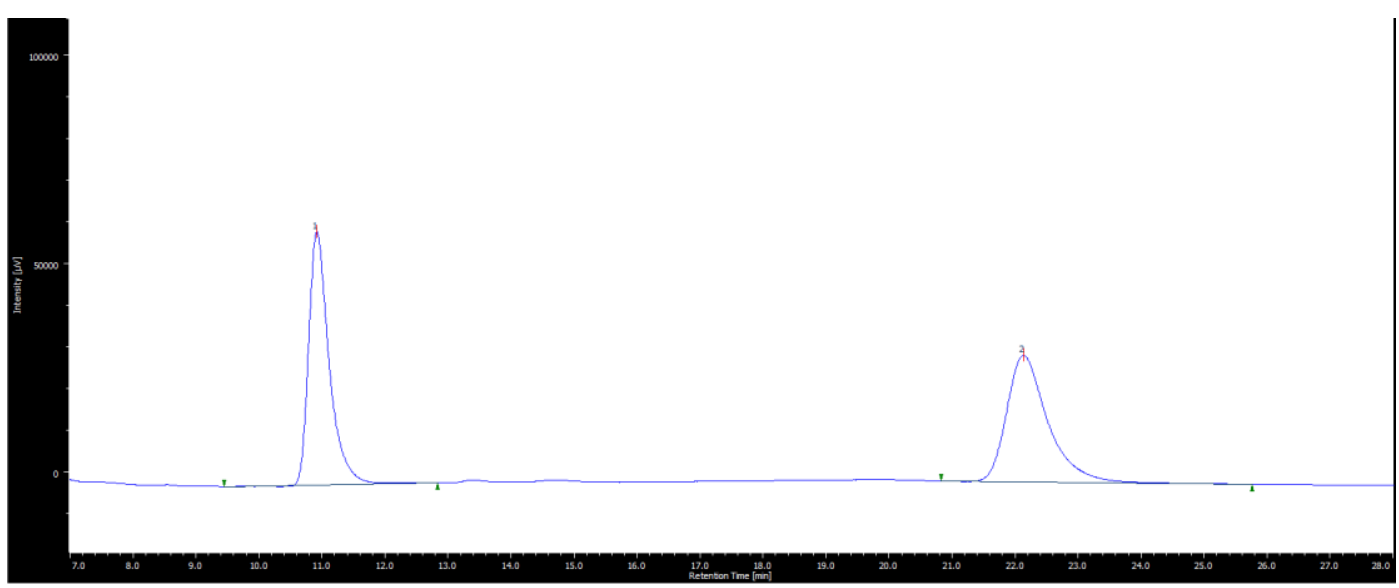

(R)-3h

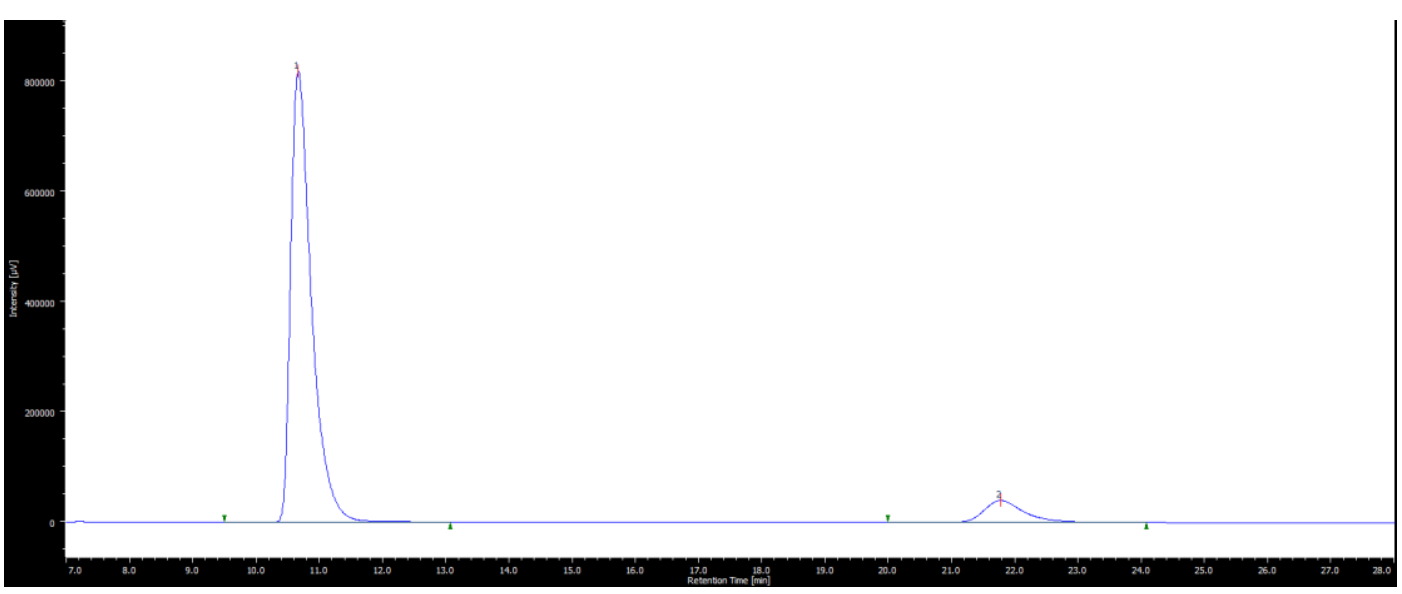

\begin{tabular}{|c|c|c|}
\hline \multicolumn{3}{|c|}{ racemic-3h } \\
\hline Peak & tR (min) & Area (\%) \\
\hline 1 & 10.9 & 49.9 \\
\hline 2 & 22.1 & 50.1 \\
\hline
\end{tabular}

\begin{tabular}{|c|c|c|}
\hline \multicolumn{3}{|c|}{$(R)-3 h$} \\
\hline Peak & tR (min) & Area (\%) \\
\hline 1 & 10.7 & 91.4 \\
\hline 2 & 21.8 & 8.6 \\
\hline
\end{tabular}


Methyl (R,E)-4-[1-benzyl-3-(benzyloxycarbonylamino)-7-trifluoromethyl-2-oxoindolin-3-yl]but-2enoate (3i)

racemic-3i<smiles>COC(=O)/C=C/C[C@@]1(NC(=O)OCc2ccccc2)C(=O)N(Cc2ccccc2)c2c(C)cccc21</smiles>

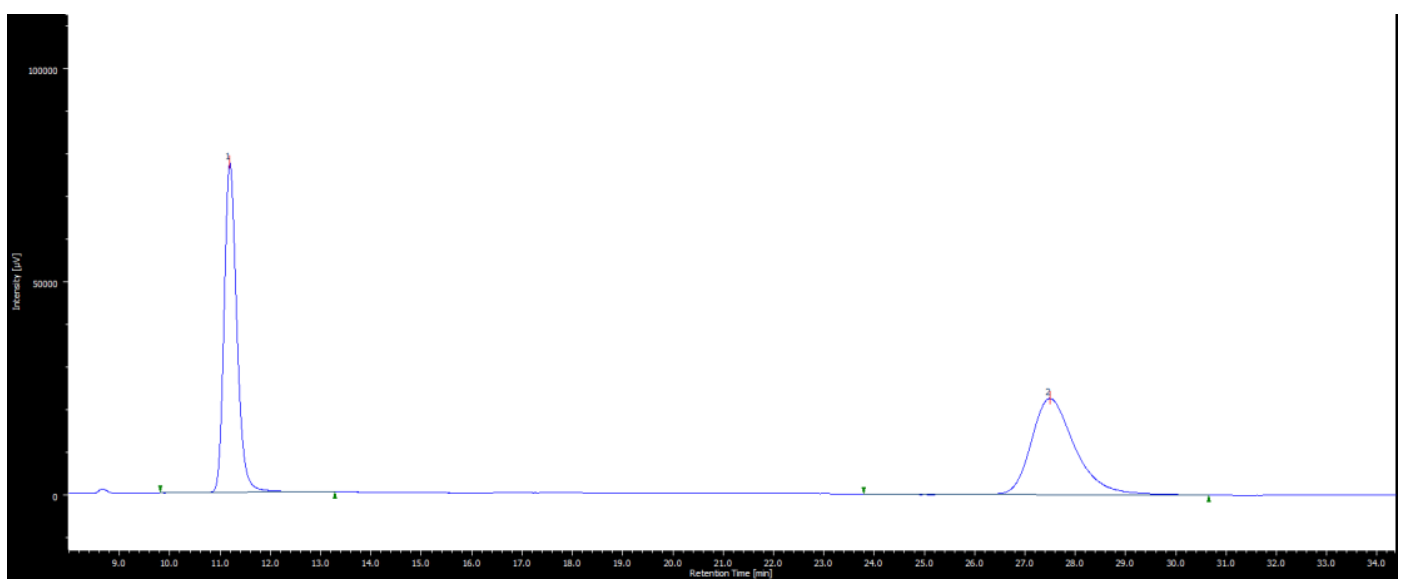

(R)-3i

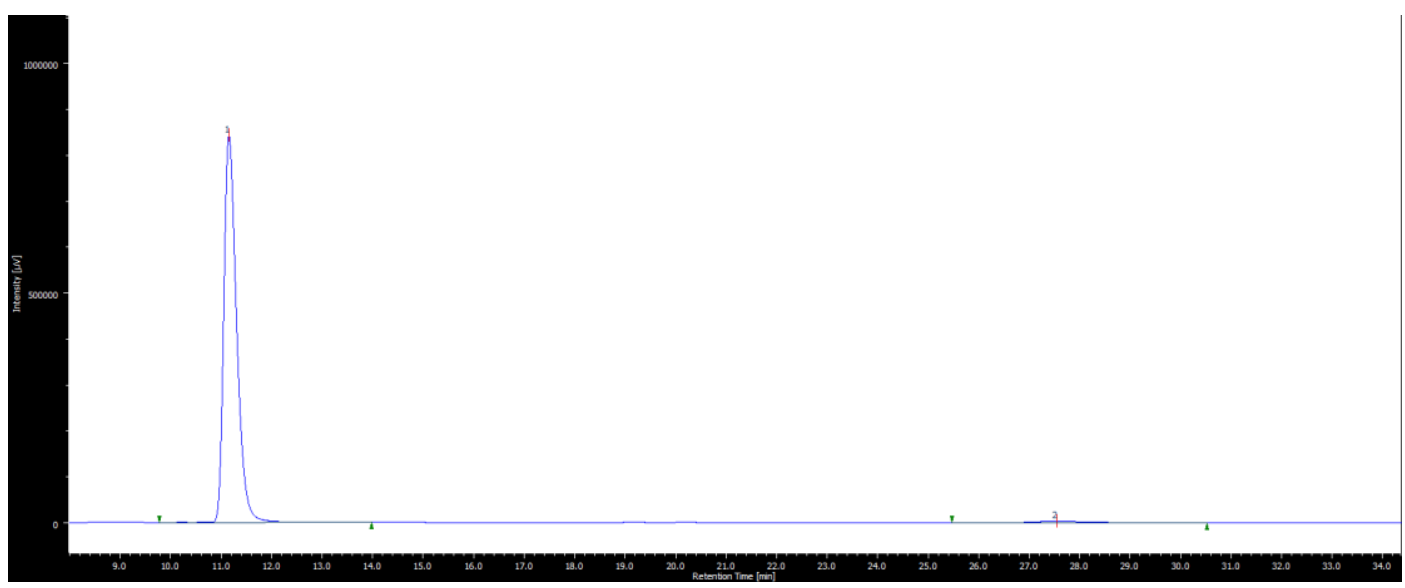

\begin{tabular}{|c|c|c|}
\hline \multicolumn{3}{|c|}{ racemic-3i } \\
\hline Peak & tR (min) & Area (\%) \\
\hline 1 & 11.2 & 50.2 \\
\hline 2 & 27.5 & 49.8 \\
\hline
\end{tabular}

\begin{tabular}{|c|c|c|}
\hline \multicolumn{3}{|c|}{$(R)-3 i$} \\
\hline Peak & tR (min) & Area (\%) \\
\hline 1 & 13.1 & 98.7 \\
\hline 2 & 35.1 & 1.3 \\
\hline
\end{tabular}


Methyl (R,E)-4-[1-benzyl-3-(benzyloxycarbonylamino)-5-chloro-2-oxoindolin-3-yl]but-2-enoate (3j)

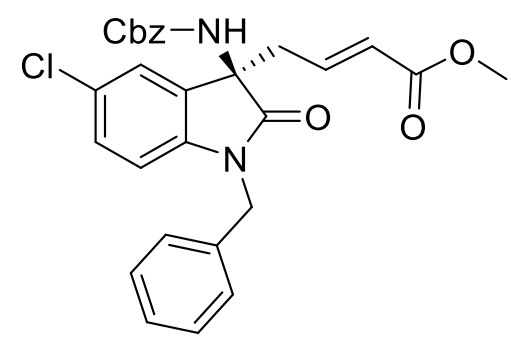

racemic-3j

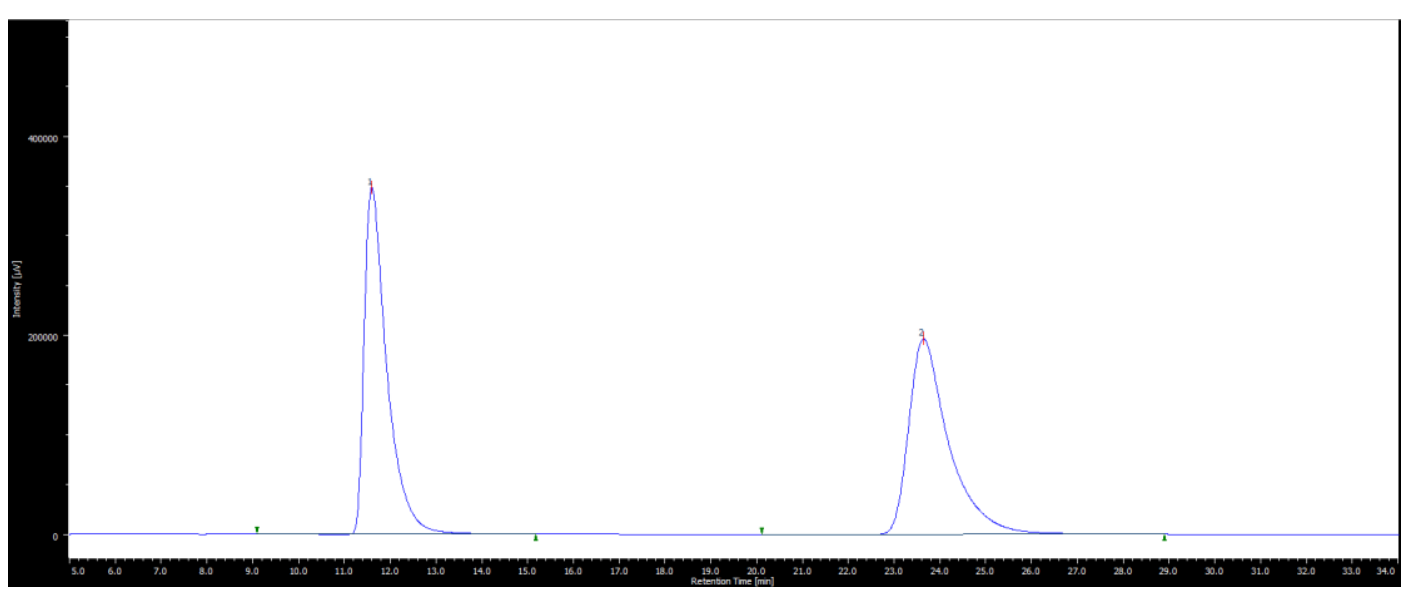

(R)-3j

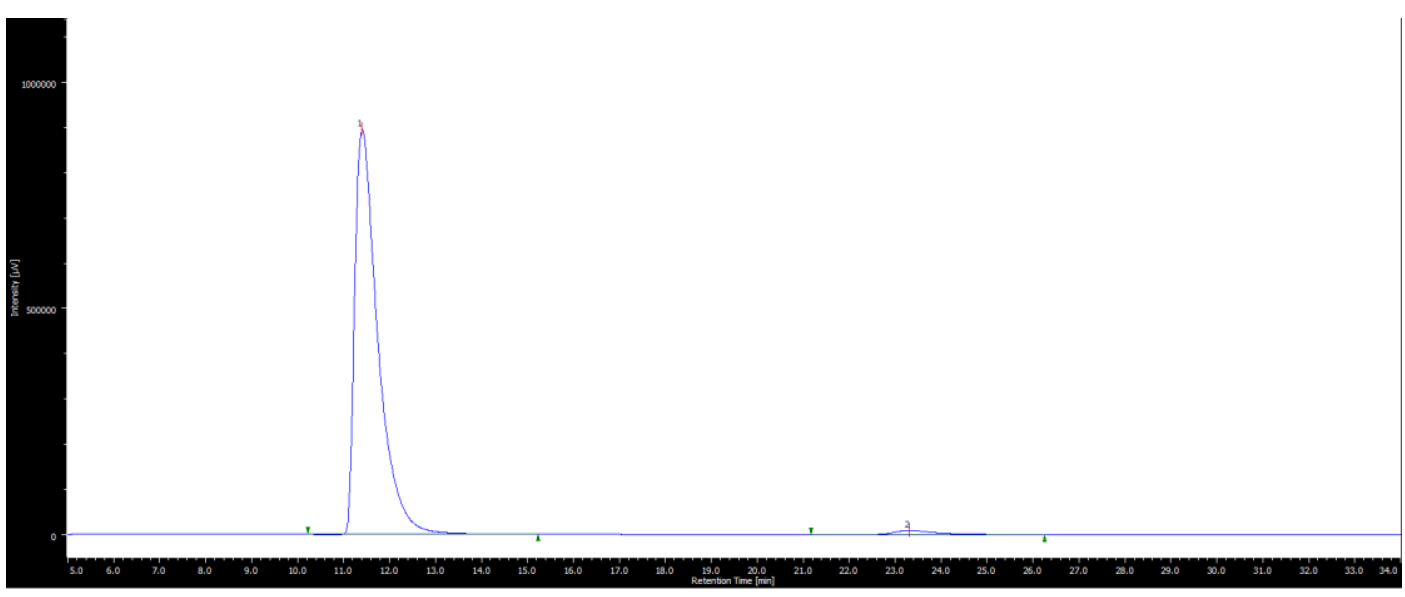

\begin{tabular}{|c|c|c|}
\hline \multicolumn{3}{|c|}{ racemic-3j } \\
\hline Peak & tR (min) & Area (\%) \\
\hline 1 & 11.6 & 50.1 \\
\hline 2 & 23.6 & 49.9 \\
\hline
\end{tabular}

\begin{tabular}{|c|c|c|}
\hline \multicolumn{3}{|c|}{$(R)-3 \mathbf{j}$} \\
\hline Peak & tR (min) & Area (\%) \\
\hline 1 & 11.4 & 98.3 \\
\hline 2 & 23.3 & 1.7 \\
\hline
\end{tabular}


Methyl (R,E)-4-[1-benzyl-3-(benzyloxycarbonylamino)-5-nitro-2-oxoindolin-3-yl]but-2-enoate $(3 \mathrm{k})$

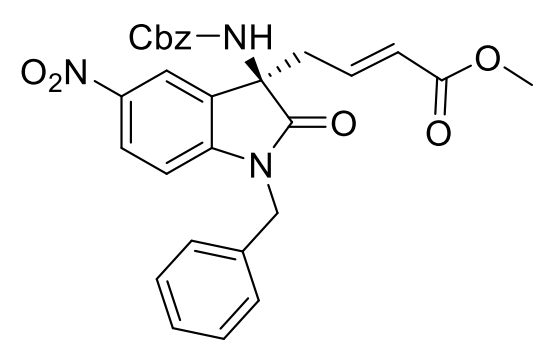

racemic-3k

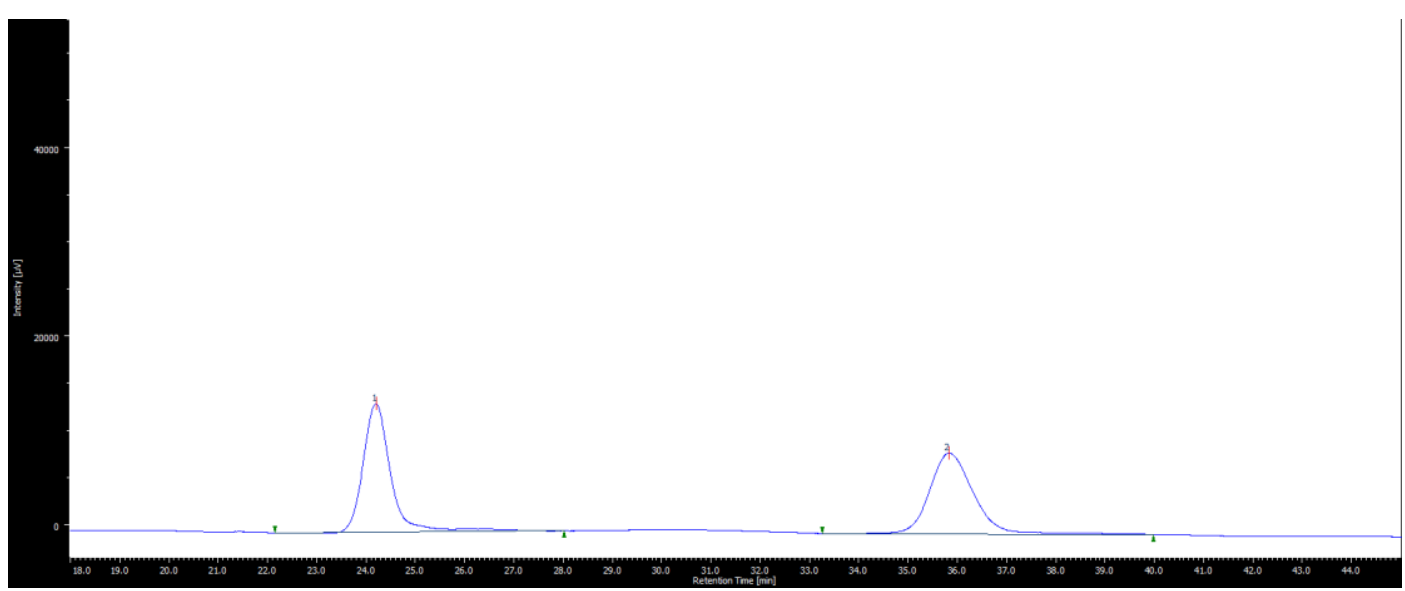

$(R)-3 \mathbf{k}$

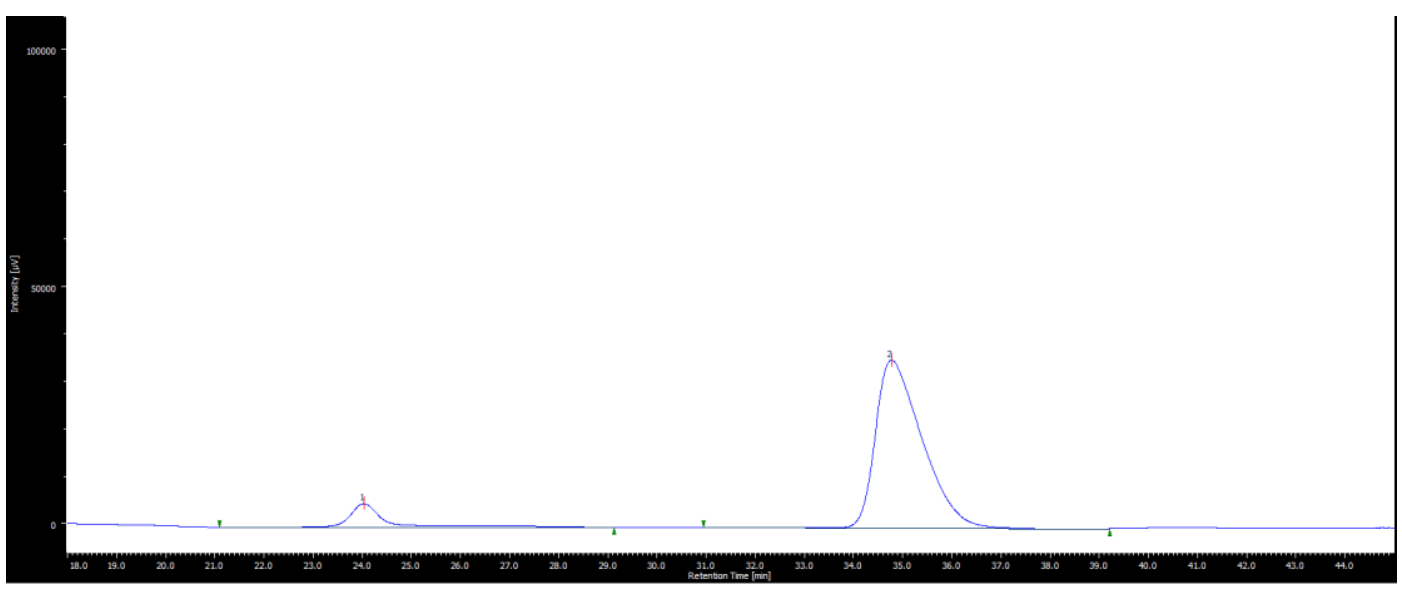

\begin{tabular}{|c|c|c|}
\hline \multicolumn{3}{|c|}{ racemic-3k } \\
\hline Peak & tR (min) & Area (\%) \\
\hline 1 & 24.2 & 50.1 \\
\hline 2 & 35.8 & 49.9 \\
\hline
\end{tabular}

\begin{tabular}{|c|c|c|}
\hline \multicolumn{3}{|c|}{$(R)-3 k$} \\
\hline Peak & tR (min) & Area (\%) \\
\hline 1 & 24.0 & 10.4 \\
\hline 2 & 34.8 & 89.6 \\
\hline
\end{tabular}


Methyl (R,E)-4-[1-benzyl-3-(benzyloxycarbonylamino)-5-methyl-2-oxoindolin-3-yl]but-2-enoate (3I)

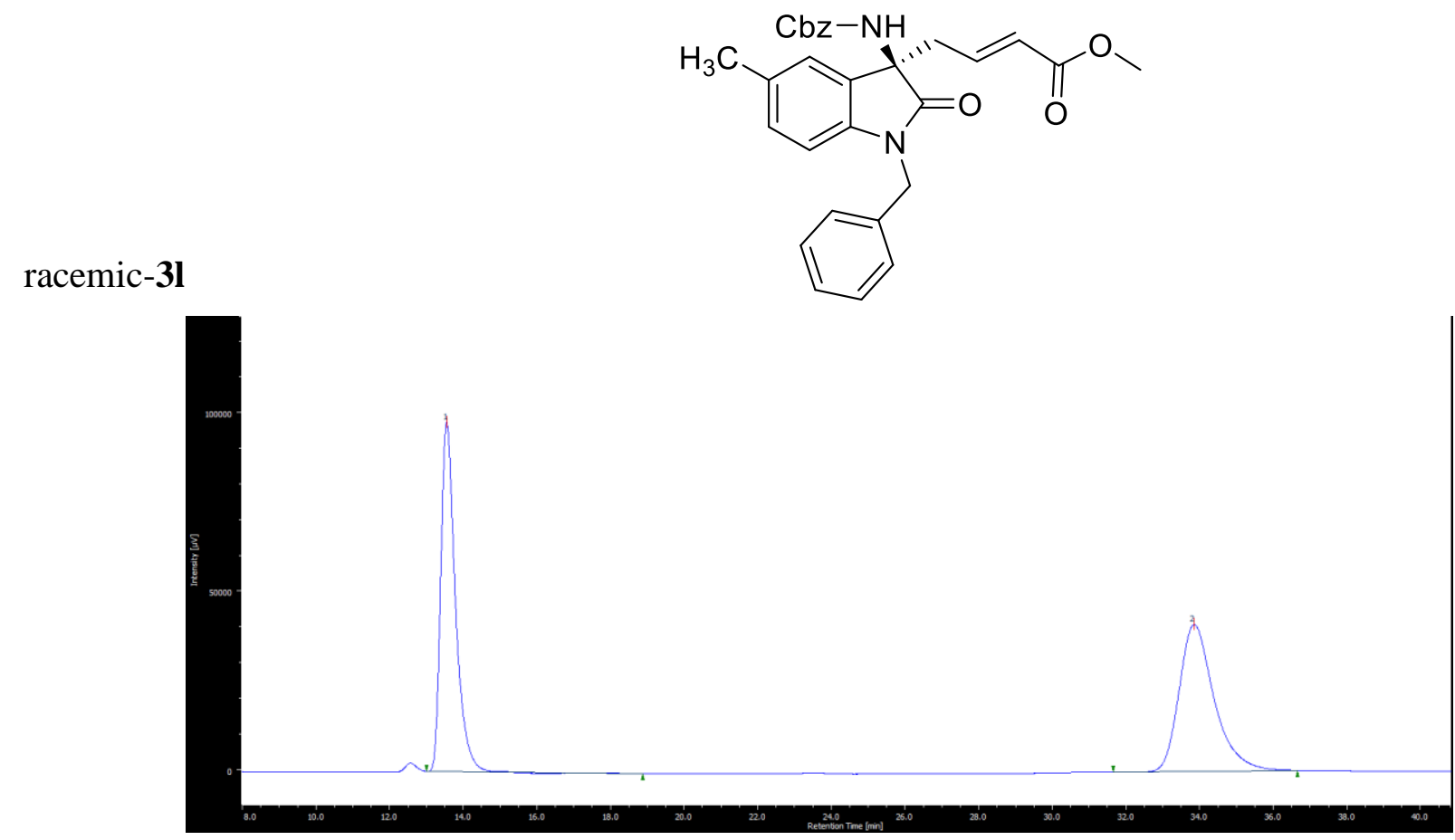

$(R)-31$

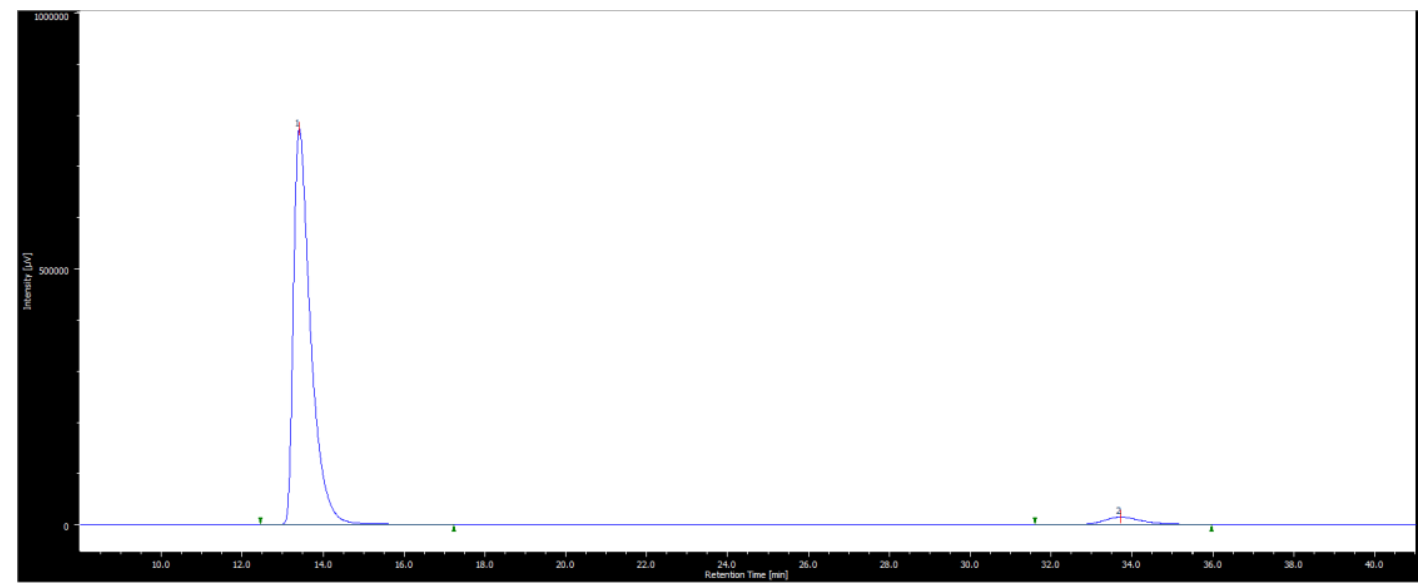

\begin{tabular}{|c|c|c|}
\hline \multicolumn{3}{|c|}{ racemic-3l } \\
\hline Peak & tR (min) & Area (\%) \\
\hline 1 & 13.6 & 50.0 \\
\hline 2 & 33.8 & 50.0 \\
\hline
\end{tabular}

\begin{tabular}{|c|c|c|}
\hline \multicolumn{3}{|c|}{$(R)-31$} \\
\hline Peak & tR (min) & Area (\%) \\
\hline 1 & 13.4 & 96.0 \\
\hline 2 & 33.7 & 4.0 \\
\hline
\end{tabular}


Methyl (R,E)-4-[1-benzyl-3-(benzyloxycarbonylamino)-5-methoxy-2-oxoindolin-3-yl]but-2-enoate (3m)

racemic-3m
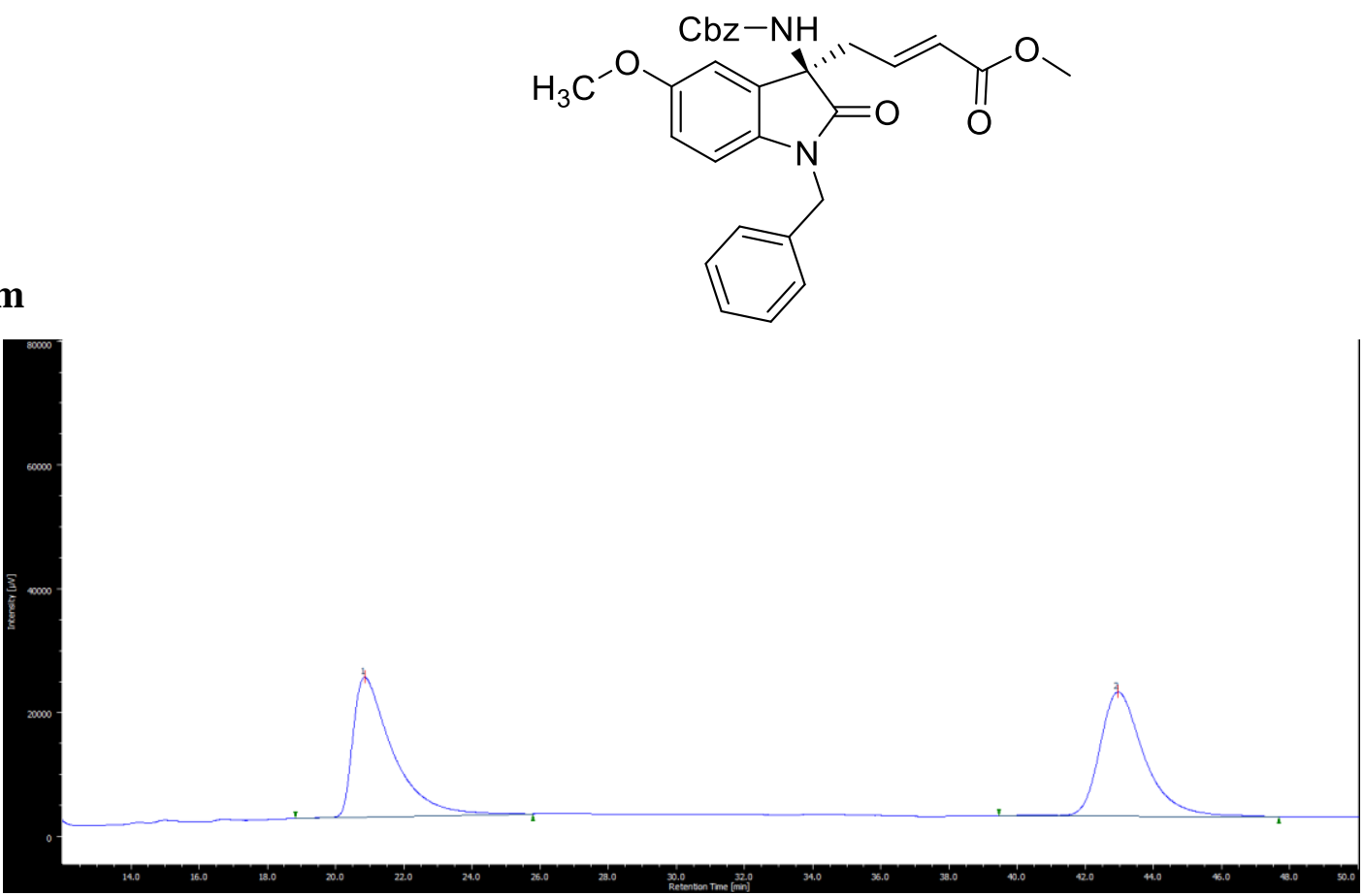

(R)-3m

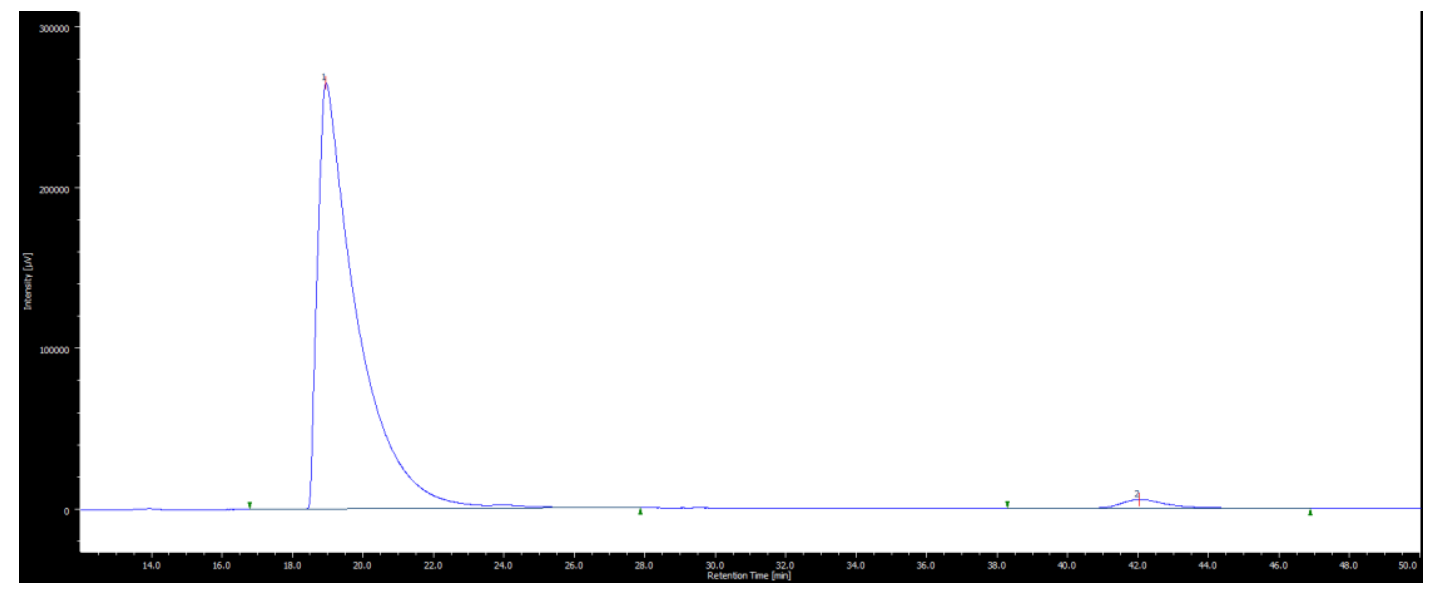

\begin{tabular}{|c|c|c|}
\hline \multicolumn{3}{|c|}{ racemic-3m } \\
\hline Peak & tR (min) & Area (\%) \\
\hline 1 & 20.9 & 50.3 \\
\hline 2 & 42.9 & 49.7 \\
\hline
\end{tabular}

\begin{tabular}{|c|c|c|}
\hline \multicolumn{3}{|c|}{$(R)-3 \mathbf{m}$} \\
\hline Peak & tR (min) & Area (\%) \\
\hline 1 & 19.0 & 97.7 \\
\hline 2 & 42.0 & 2.3 \\
\hline
\end{tabular}


Methyl (R,E)-4-[1-benzyl-3-(benzyloxycarbonylamino)-5,7-dimethyl-2-oxoindolin-3-yl]but-2enoate (3n)

racemic-3n
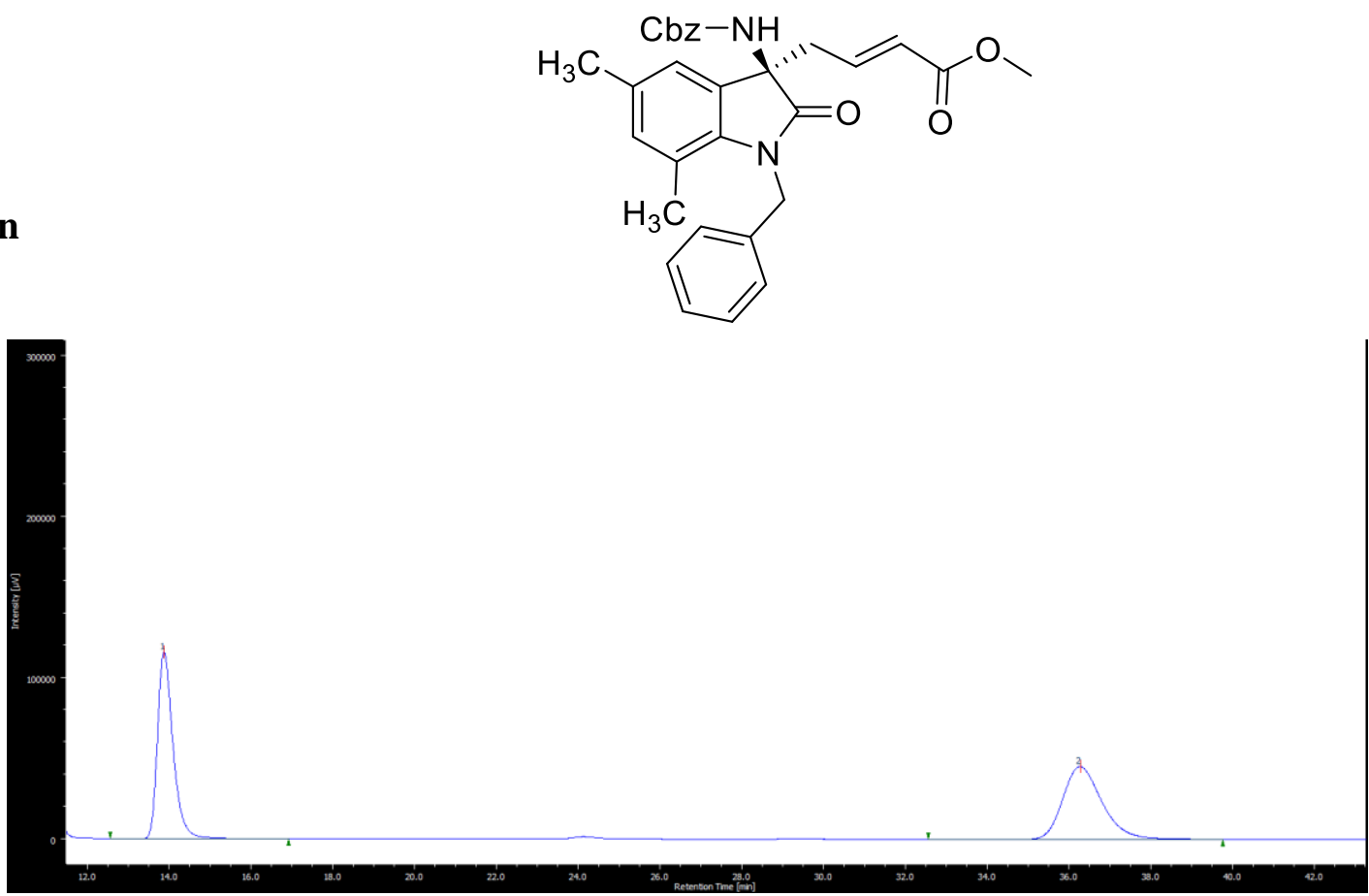

(R)-3n

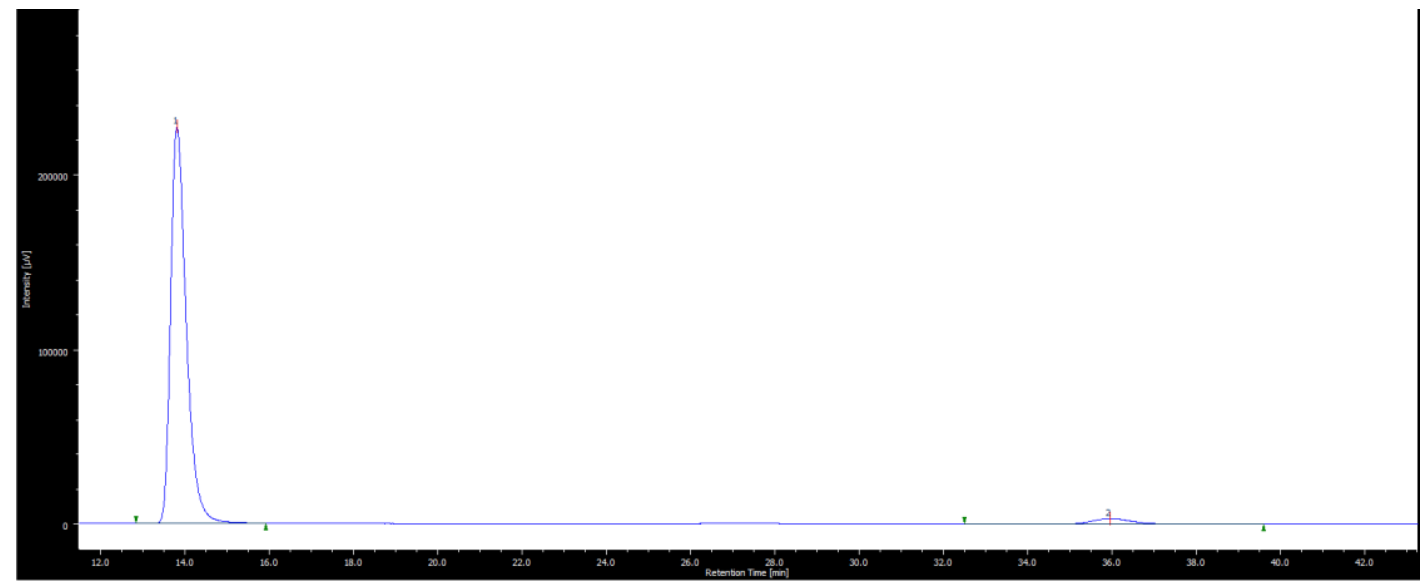

\begin{tabular}{|c|c|c|}
\hline \multicolumn{3}{|c|}{ racemic-3n } \\
\hline Peak & tR (min) & Area (\%) \\
\hline 1 & 13.8 & 50.2 \\
\hline 2 & 36.3 & 49.8 \\
\hline
\end{tabular}

\begin{tabular}{|c|c|c|}
\hline \multicolumn{3}{|c|}{$(R)-3 n$} \\
\hline Peak & tR (min) & Area (\%) \\
\hline 1 & 13.8 & 96.8 \\
\hline 2 & 35.9 & 3.2 \\
\hline
\end{tabular}


Methyl

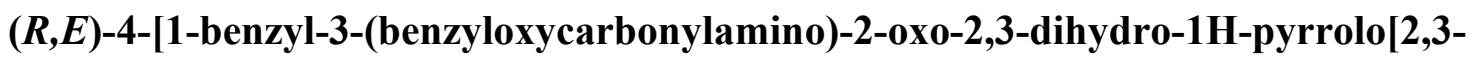

b]pyridin-3-yl]but-2-enoate (3o)

racemic-3o<smiles>COC(=O)/C=C/C[C@@]1(NC(=O)OC)C(=O)N(C)c2ncccc21</smiles>

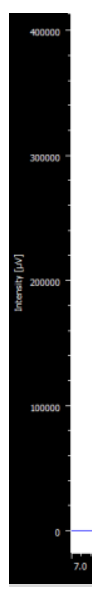

(R)-3o

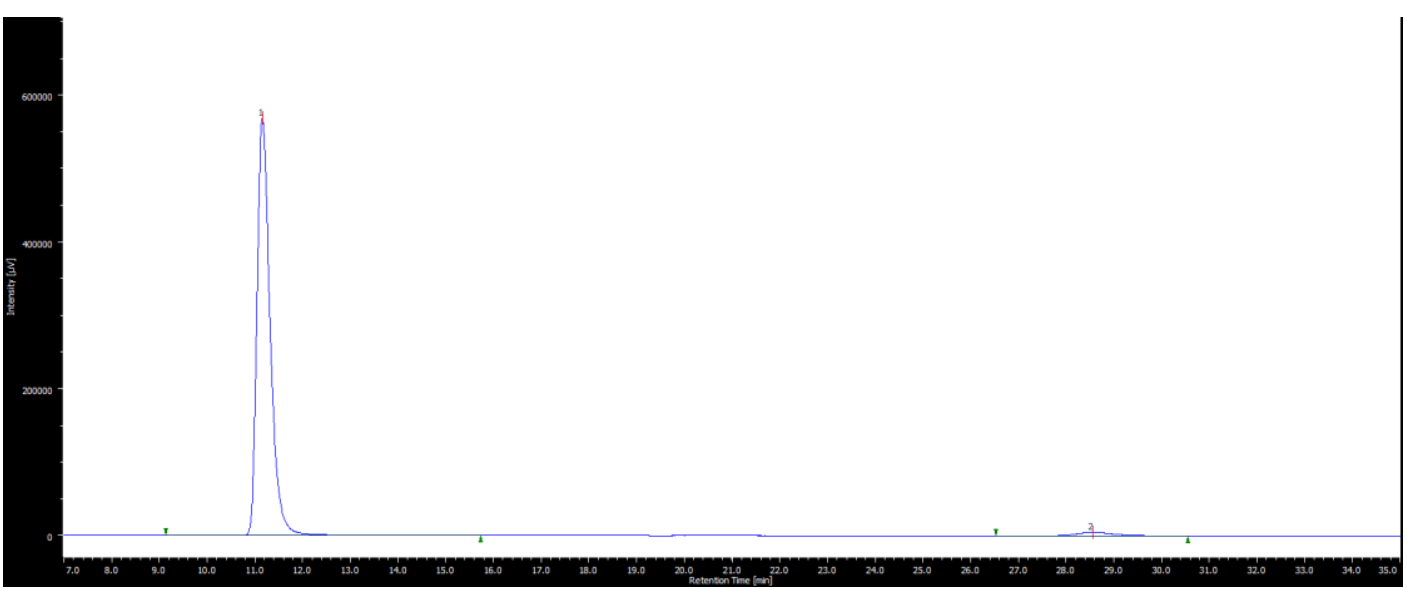

\begin{tabular}{|c|c|c|}
\hline \multicolumn{3}{|c|}{ racemic-3o } \\
\hline Peak & tR (min) & Area (\%) \\
\hline 1 & 11.2 & 50.3 \\
\hline 2 & 28.3 & 49.7 \\
\hline
\end{tabular}

\begin{tabular}{|c|c|c|}
\hline \multicolumn{3}{|c|}{$(R)-30$} \\
\hline Peak & tR (min) & Area (\%) \\
\hline 1 & 11.2 & 97.8 \\
\hline 2 & 28.6 & 2.2 \\
\hline
\end{tabular}


Methyl (R,E)-4-[1-benzyl-3-(benzyloxycarbonylamino)-2-oxoindolin-3-yl]-2-methylbut-2-enoate (3p)

racemic-3p<smiles>COC(=O)/C(C)=C/C[C@@]1(NC(=O)OCc2ccccc2)C(=O)Nc2ccccc21</smiles>

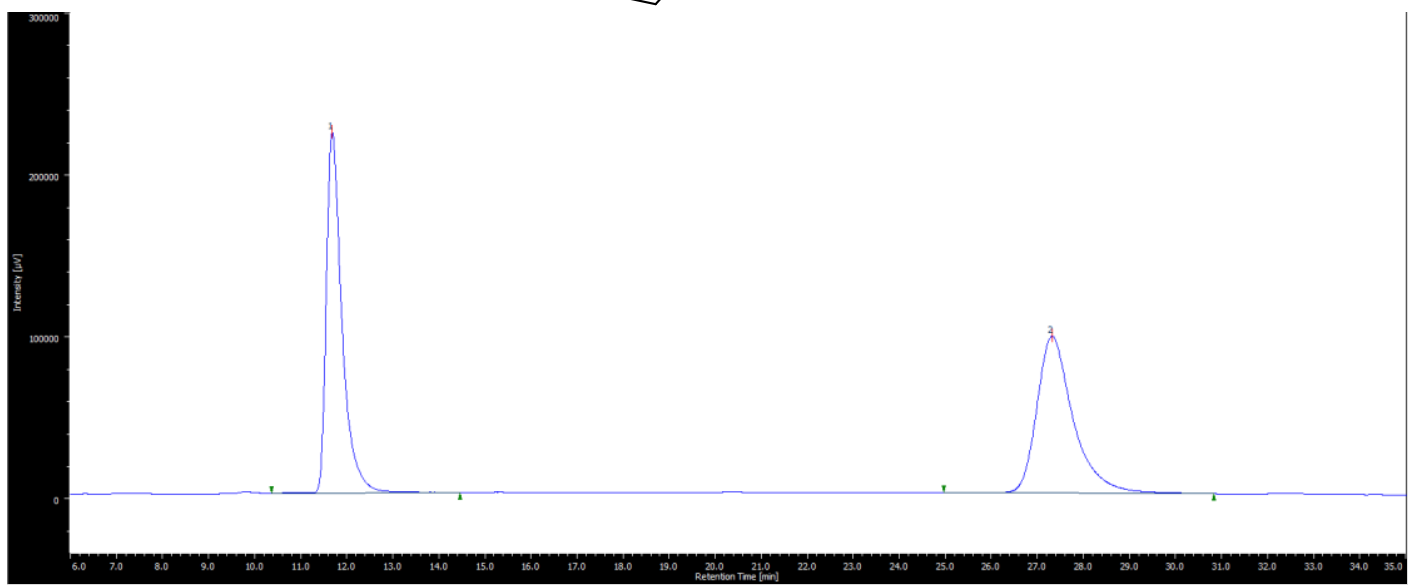

$(R)-3 \mathbf{p}$

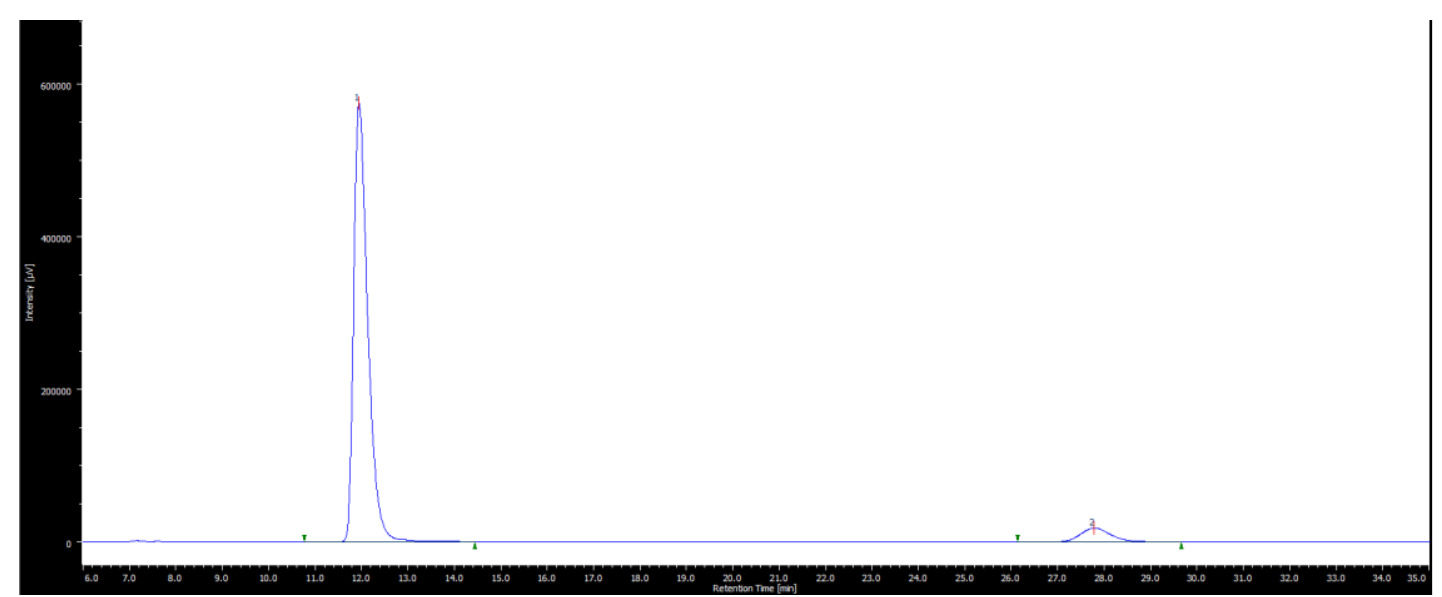

\begin{tabular}{|c|c|c|}
\hline \multicolumn{3}{|c|}{ racemic-3p } \\
\hline Peak & tR (min) & Area (\%) \\
\hline 1 & 11.7 & 49.6 \\
\hline 2 & 27.3 & 50.4 \\
\hline
\end{tabular}

\begin{tabular}{|c|c|c|}
\hline \multicolumn{3}{|c|}{$(R)-3 p$} \\
\hline Peak & tR (min) & Area (\%) \\
\hline 1 & 11.9 & 93.6 \\
\hline 2 & 27.8 & 6.4 \\
\hline
\end{tabular}


Benzyl

(R)-[1-benzyl-3-(2,2-dimethyl-4-oxo-4H-1,3-dioxin-6-yl)methyl)-2-oxoindolin-3-

yl]carbamate (3q)

racemic-3q
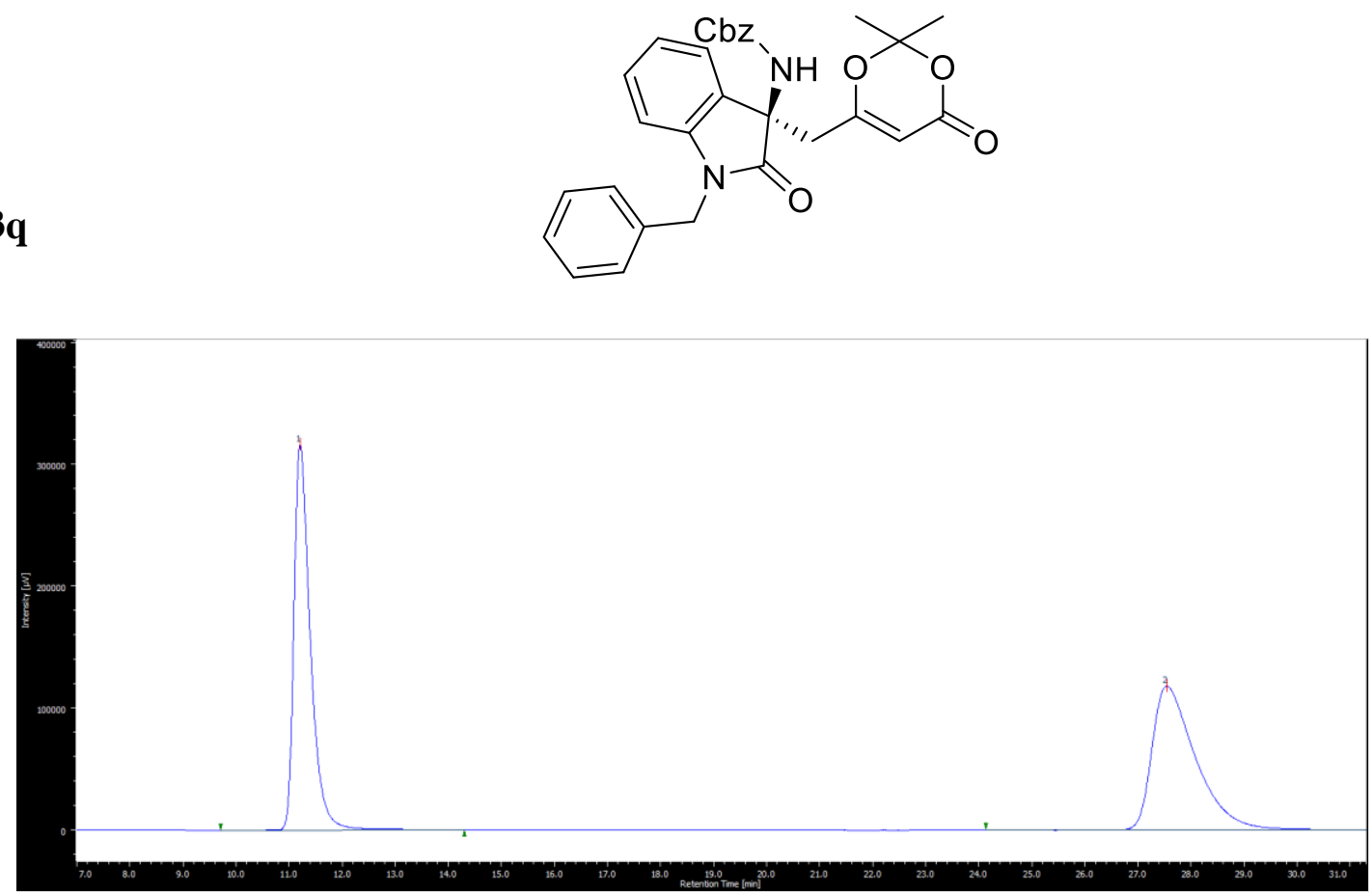

(R)-3q

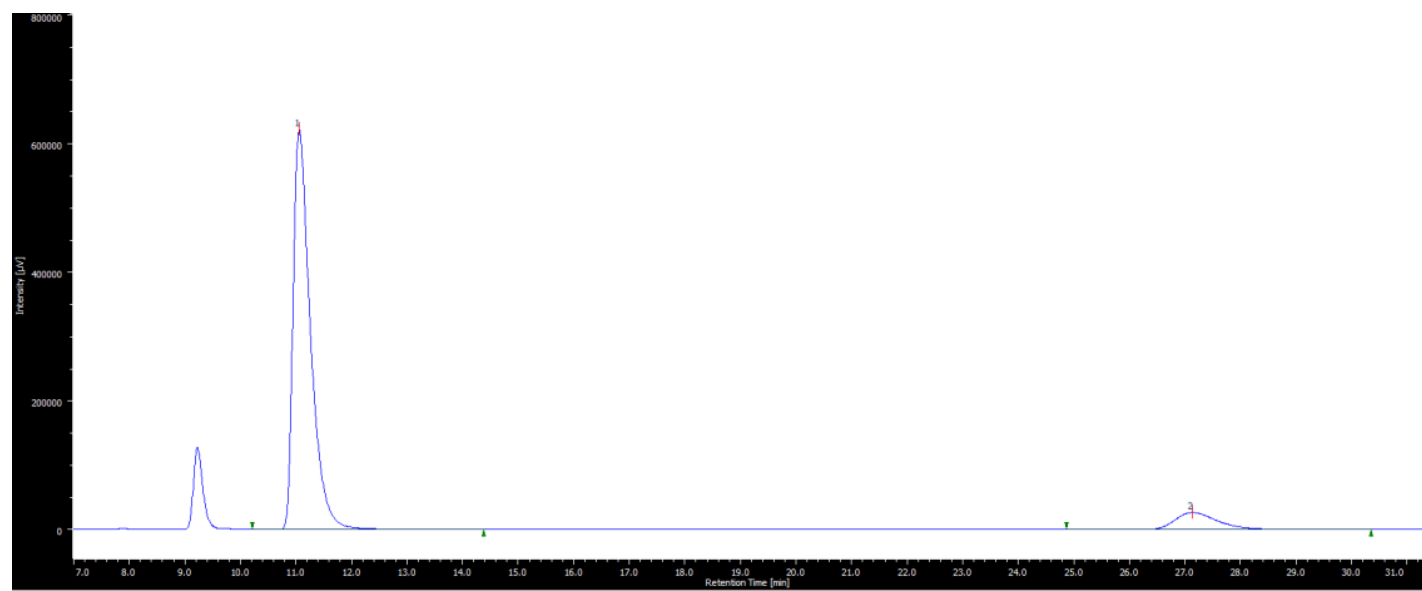

\begin{tabular}{|c|c|c|}
\hline \multicolumn{3}{|c|}{ racemic-3q } \\
\hline Peak & tR (min) & Area (\%) \\
\hline 1 & 11.2 & 50.1 \\
\hline 2 & 27.5 & 49.9 \\
\hline
\end{tabular}

\begin{tabular}{|c|c|c|}
\hline \multicolumn{3}{|c|}{$(R)-3 q$} \\
\hline Peak & tR (min) & Area (\%) \\
\hline 1 & 11.1 & 90.4 \\
\hline 2 & 27.1 & 9.6 \\
\hline
\end{tabular}


Methyl (E)-4-[(R)-1-benzyl-3-(benzyloxycarbonylamino)-2-oxoindolin-3-yl]-pent-2-enoate (3r)

racemic-3r<smiles>COC(=O)/C=C/[C@@H](C)[C@@]1(NC(=O)OCc2ccccc2)C(=O)Nc2ccccc21</smiles>

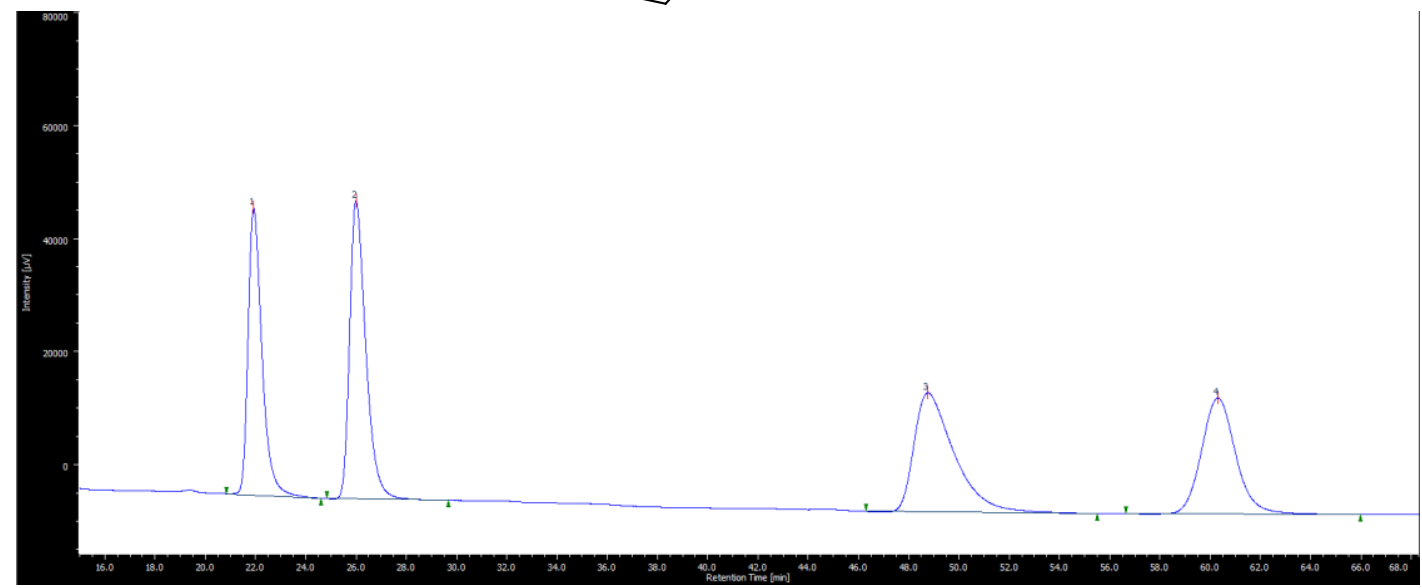

(R)-3r

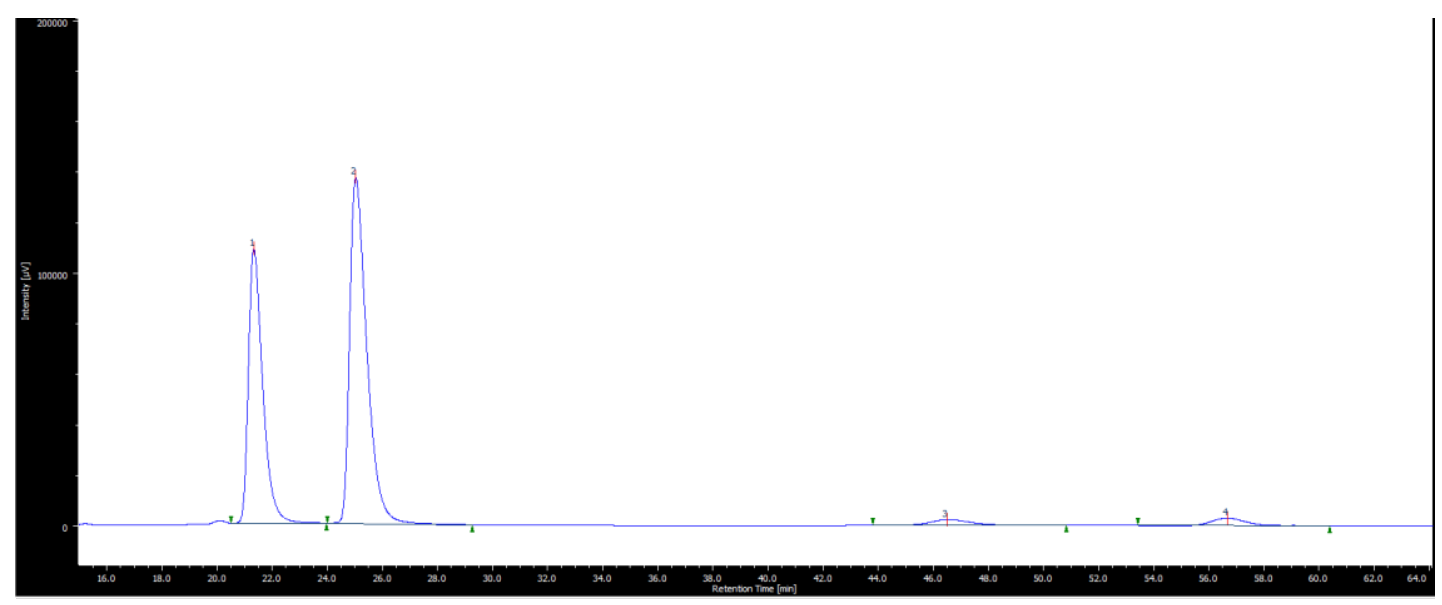

\begin{tabular}{|c|c|c||c|c|c|}
\hline \multicolumn{6}{|c|}{ racemic-3r } \\
\hline Peak & $\begin{array}{c}\text { tR } \\
(\mathrm{min})\end{array}$ & $\begin{array}{c}\text { Area } \\
(\%)\end{array}$ & Peak & $\begin{array}{c}\text { tR } \\
(\mathrm{min})\end{array}$ & $\begin{array}{c}\text { Area } \\
(\%)\end{array}$ \\
\hline 1 & 21.9 & 22.7 & 2 & 26.0 & 27.5 \\
\hline 3 & 48.7 & 27.2 & 4 & 60.3 & 22.5 \\
\hline
\end{tabular}

\begin{tabular}{|c|c|c||c|c|c|}
\hline \multicolumn{6}{|c|}{ chiral-3r } \\
\hline Peak & $\begin{array}{c}\text { tR } \\
(\mathrm{min})\end{array}$ & $\begin{array}{c}\text { Area } \\
(\%)\end{array}$ & Peak & $\begin{array}{c}\text { tR } \\
(\mathrm{min})\end{array}$ & $\begin{array}{c}\text { Area } \\
(\%)\end{array}$ \\
\hline 1 & 21.3 & 38.1 & 2 & 25.0 & 57.3 \\
\hline 3 & 46.5 & 2.1 & 4 & 56.7 & 2.5 \\
\hline
\end{tabular}


Methyl (R)-6-(3-amino-1-benzyl-2-oxoindolin-3-yl)hexanoate (5)

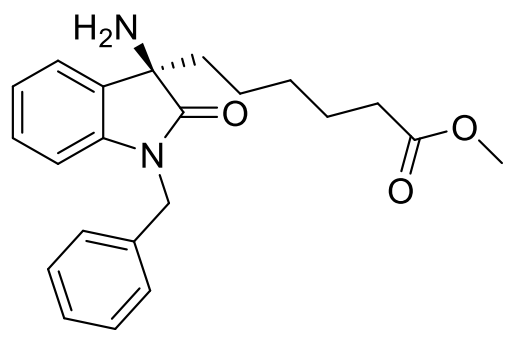

racemic-5

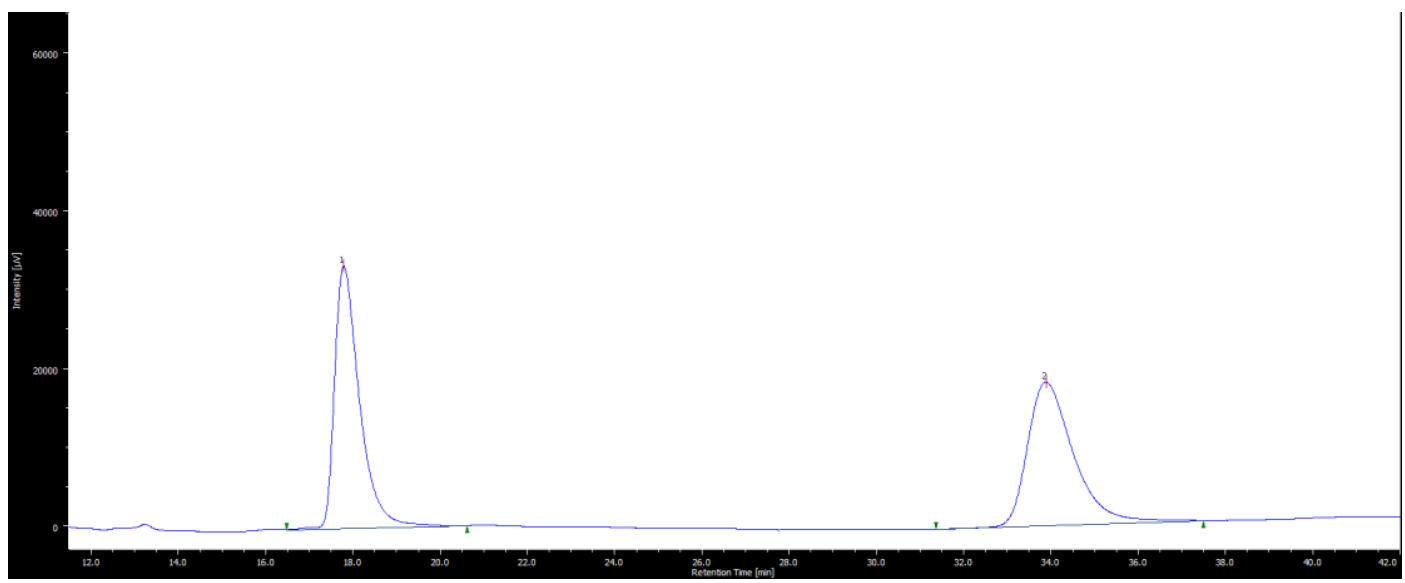

(R)-5

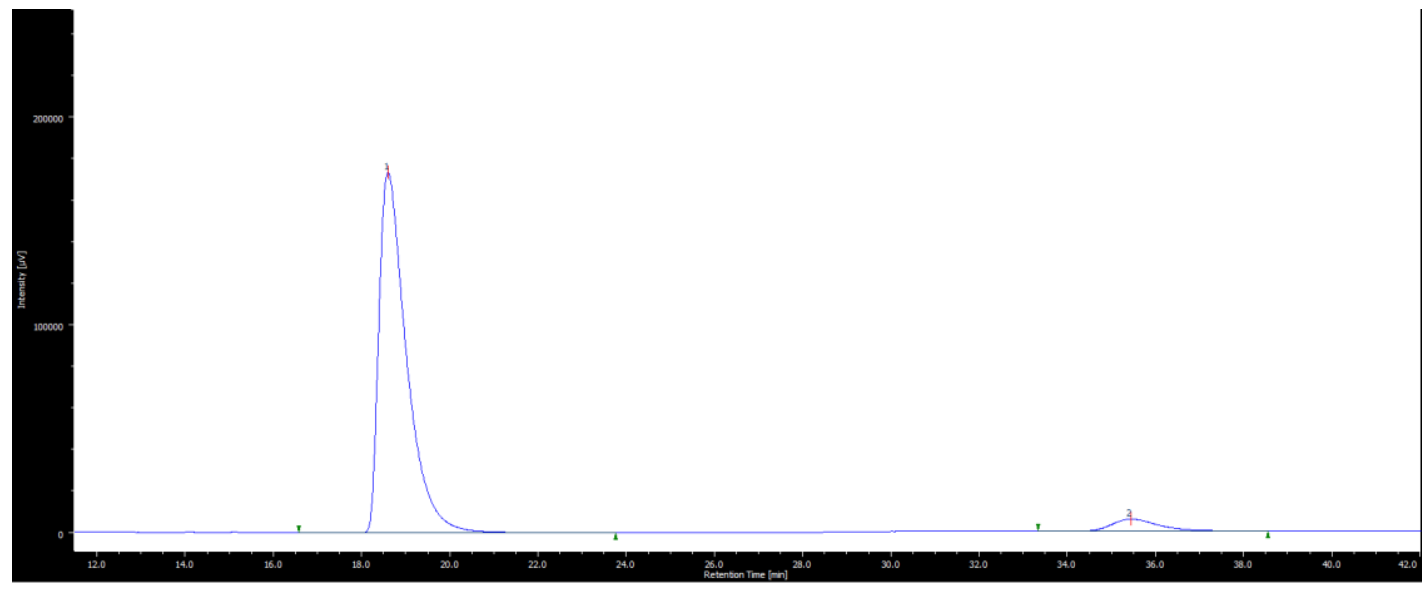

\begin{tabular}{|c|c|c|}
\hline \multicolumn{3}{|c|}{ racemic-5 } \\
\hline Peak & tR (min) & Area (\%) \\
\hline 1 & 17.8 & 49.8 \\
\hline 2 & 33.9 & 50.2 \\
\hline
\end{tabular}

\begin{tabular}{|c|c|c|}
\hline \multicolumn{3}{|c|}{$(R)-5$} \\
\hline Peak & tR (min) & Area (\%) \\
\hline 1 & 18.6 & 94.5 \\
\hline 2 & 35.4 & 5.5 \\
\hline
\end{tabular}


(R)-1-Benzylspiro[indoline-3,2'-piperidine]-2,6'-dione (7)

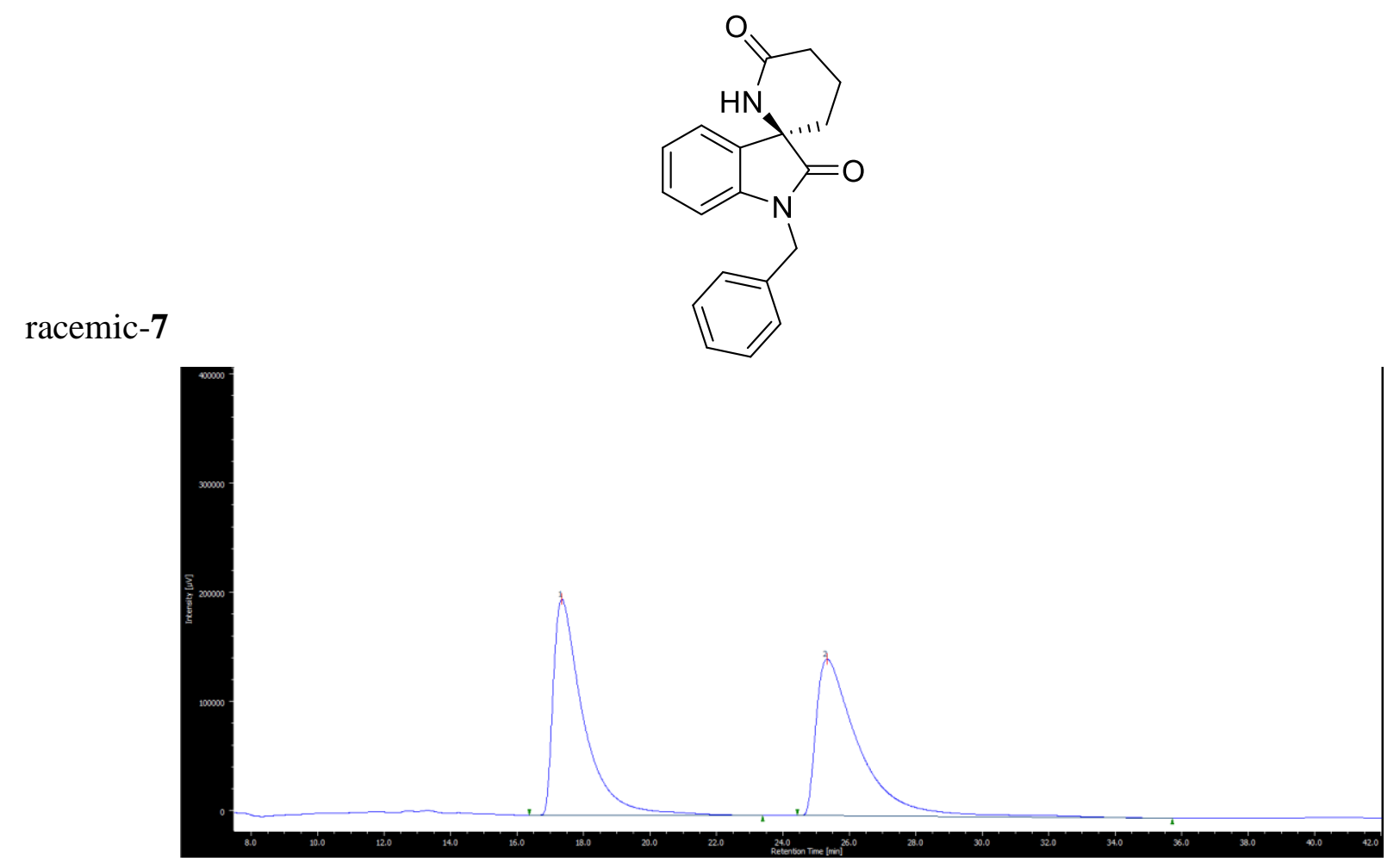

$(R)-7$

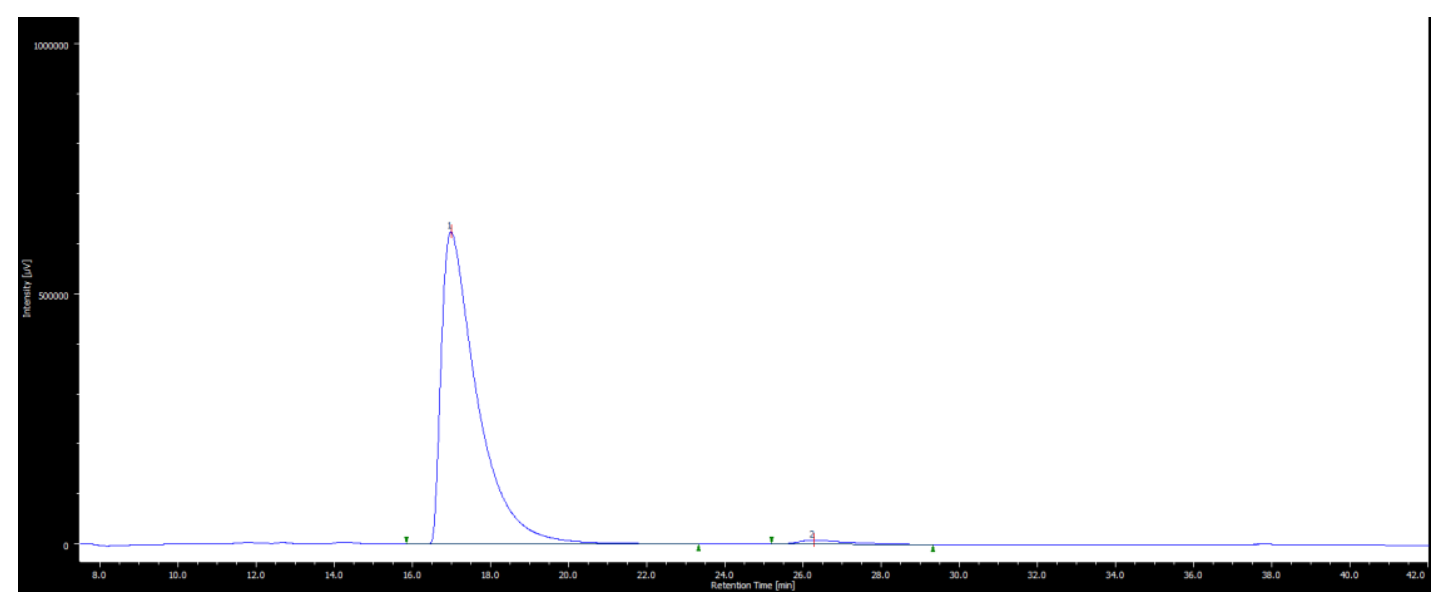

\begin{tabular}{|c|c|c|}
\hline \multicolumn{3}{|c|}{ racemic-7 } \\
\hline Peak & tR (min) & Area (\%) \\
\hline 1 & 17.3 & 49.2 \\
\hline 2 & 25.3 & 50.8 \\
\hline
\end{tabular}

\begin{tabular}{|c|c|c|}
\hline \multicolumn{3}{|c|}{$(R)-7$} \\
\hline Peak & tR (min) & Area (\%) \\
\hline 1 & 17.0 & 98.4 \\
\hline 2 & 25.3 & 1.6 \\
\hline
\end{tabular}


Methyl (R,E)-4-[1-benzyl-5-bromo-3-(4-methoxybenzenesulfonamide)-2-oxoindolin-3-yl]but-2enoate (9)

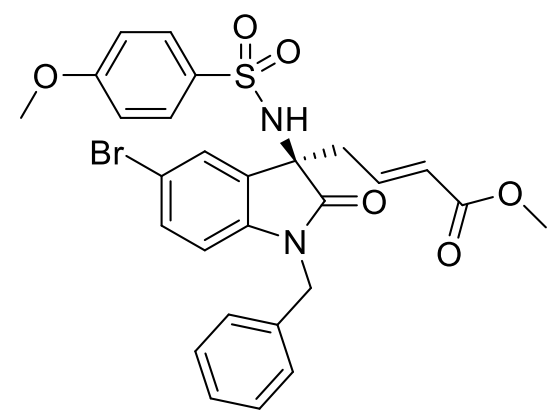

racemic-9

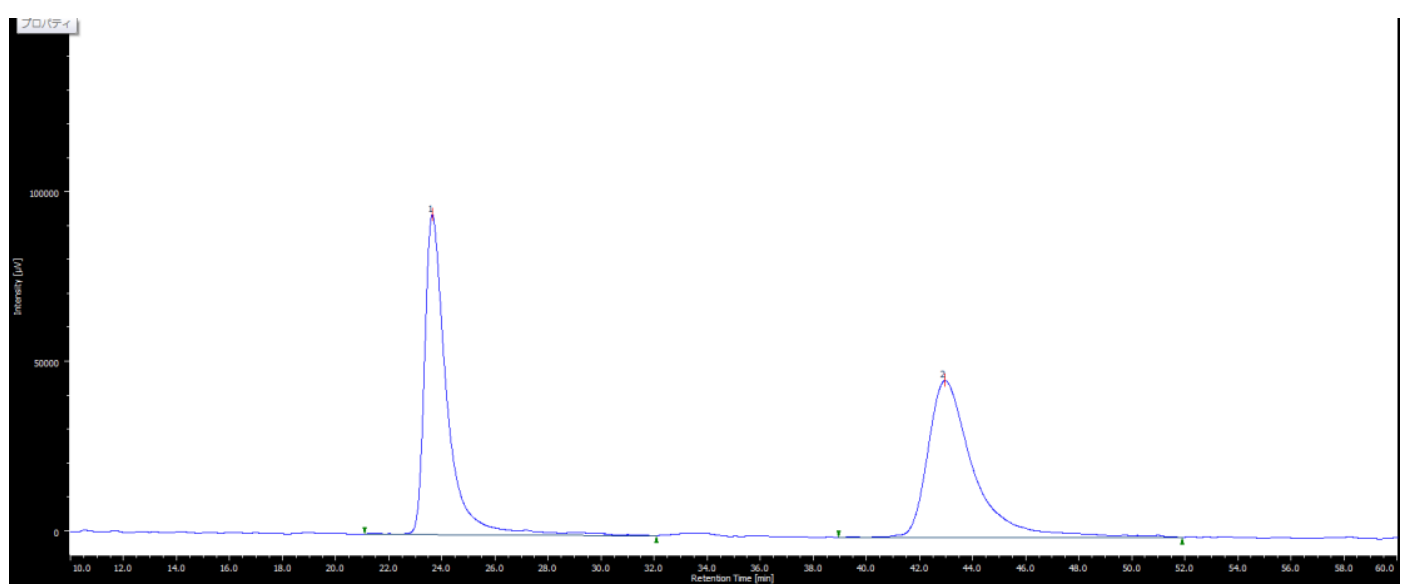

(R)-9

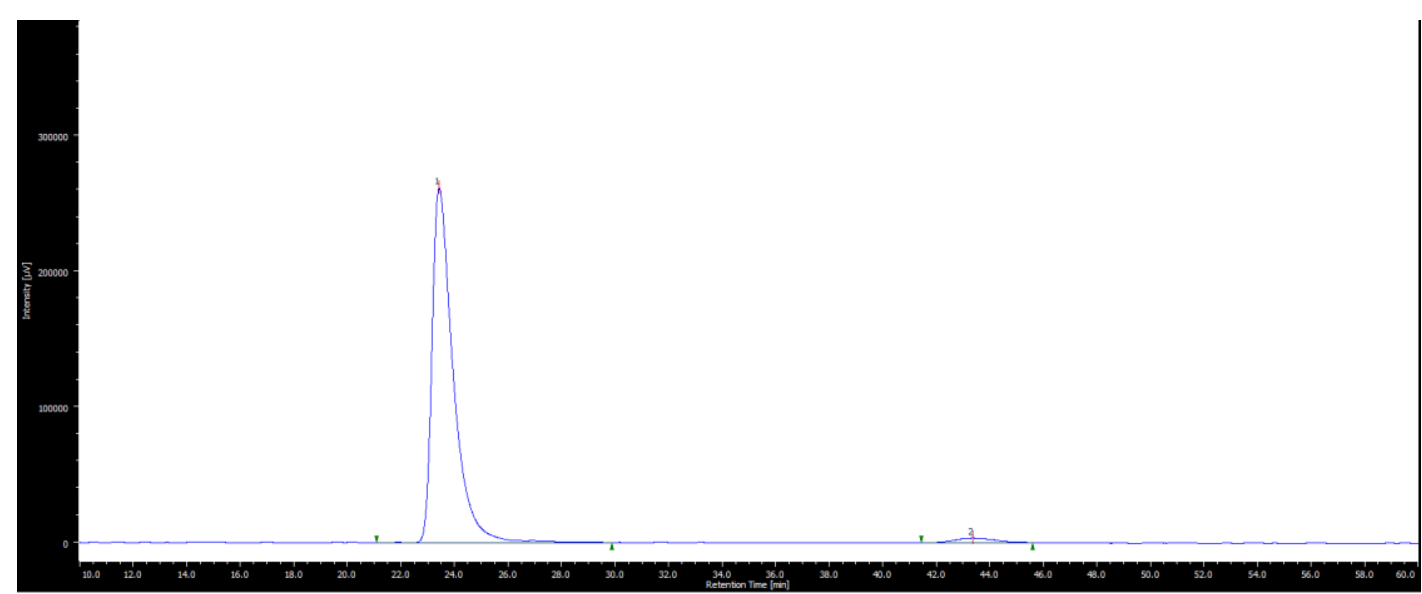

\begin{tabular}{|c|c|c|}
\hline \multicolumn{3}{|c|}{ racemic-9 } \\
\hline Peak & tR (min) & Area (\%) \\
\hline 1 & 23.6 & 49.6 \\
\hline 2 & 43.0 & 50.4 \\
\hline
\end{tabular}

\begin{tabular}{|c|c|c|}
\hline \multicolumn{3}{|c|}{$(R)-9$} \\
\hline Peak & tR (min) & Area (\%) \\
\hline 1 & 23.4 & 97.5 \\
\hline 2 & 43.4 & 2.5 \\
\hline
\end{tabular}

